\title{
Observations of Protoplanetary Disk Structures
}

\section{Sean M. Andrews}

Center for Astrophysics | Harvard \& Smithsonian

Cambridge, Massachusetts, USA 02138; email: sandrews@cfa.harvard.edu

Annu. Rev. Astron. Astrophys. 2020 58:1-49

Copyright (c) 2020 by Annual Reviews. All rights reserved

submitted 2019/09/15; accepted $2019 / 11 / 15$. An edited final version will be published later in 2020 .

\section{Keywords}

protoplanetary disks, planet formation, circumstellar matter

\section{Abstract}

The disks that orbit young stars are the essential conduits and reservoirs of material for star and planet formation. Their structures, meaning the spatial variations of the disk physical conditions, reflect the underlying mechanisms that drive those formation processes. Observations of the solids and gas in these disks, particularly at high resolution, provide fundamental insights on their mass distributions, dynamical states, and evolutionary behaviors. Over the past decade, rapid developments in these areas have largely been driven by observations with the Atacama Large Millimeter/submillimeter Array (ALMA). This review highlights the state of observational research on disk structures, emphasizing three key conclusions that reflect the main branches of the field:

- Relationships among disk structure properties are also linked to the masses, environments, and evolutionary states of their stellar hosts;

- There is clear, qualitative evidence for the growth and migration of disk solids, although the implied evolutionary timescales suggest the classical assumption of a smooth gas disk is inappropriate;

- Small-scale substructures with a variety of morphologies, locations, scales, and amplitudes - presumably tracing local gas pressure maxima - broadly influence the physical and observational properties of disks.

The last point especially is reshaping the field, with the recognition that these disk substructures likely trace active sites of planetesimal growth or are the hallmarks of planetary systems at their formation epoch. 


\section{Contents}

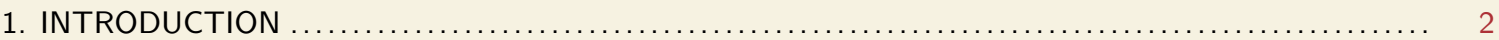

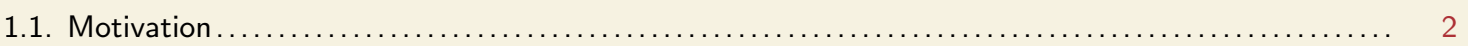

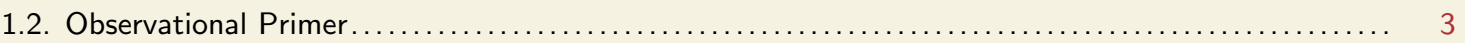

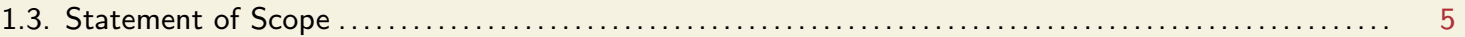

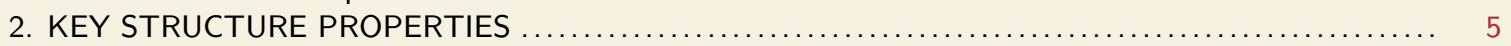

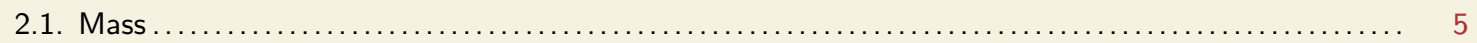

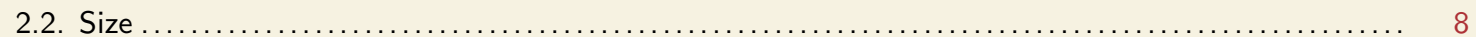

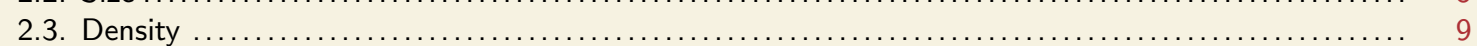

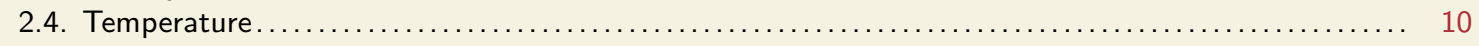

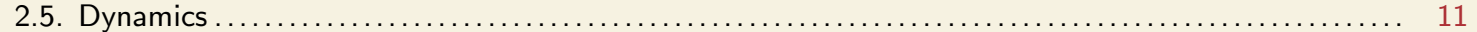

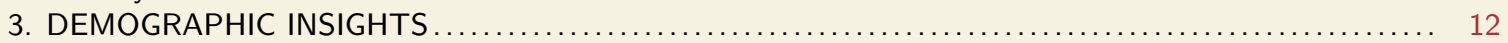

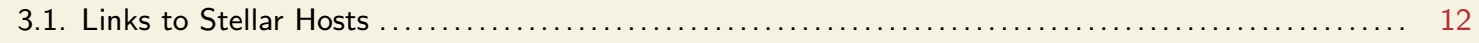

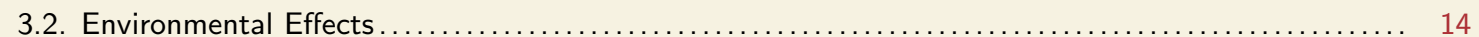

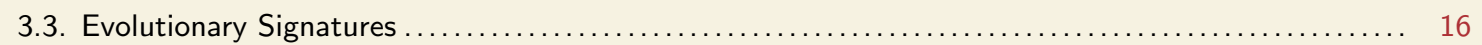

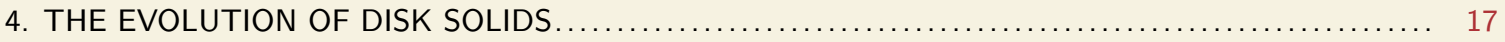

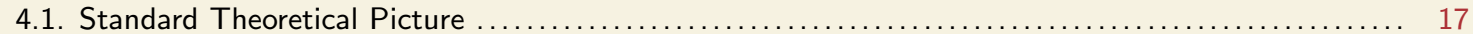

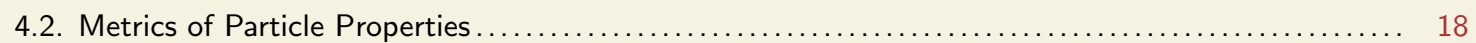

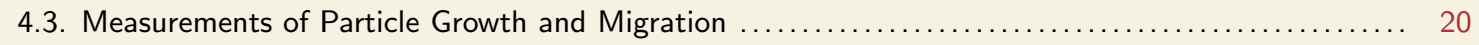

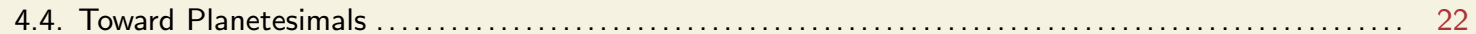

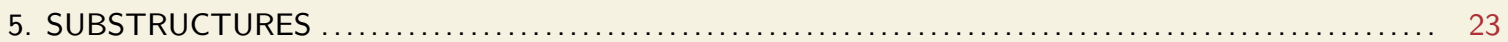

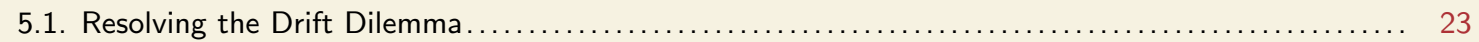

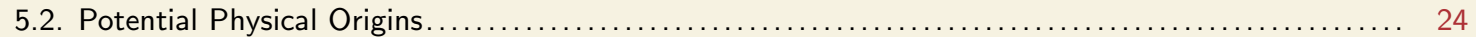

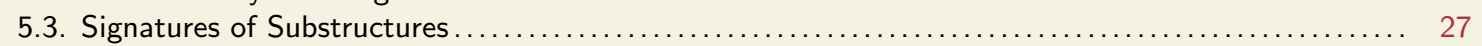

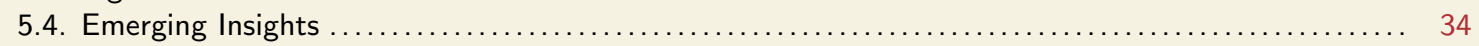

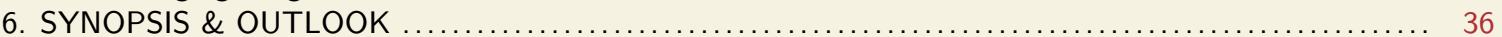

\section{INTRODUCTION}

\subsection{Motivation}

The formation and early evolution of stars and planetary systems are mediated by interactions with their circumstellar material. That material is organized in a flattened disk of gas and solids that orbits the central host star. Although these interactions between stars, planets, and disks are brief (lasting $\lesssim 10 \mathrm{Myr}$ ), they are literally foundational: such mutual influences set some stellar and planetary properties that persist for billions of years. The hallmarks of the processes that govern these links are imprinted on the disk structures, the spatial distributions and physical conditions of the disk material. Detailed observations enable measurements of those structures, their environmental dependencies, and their evolutionary behavior. Coupled with theoretical simulations and complemented by the collective knowledge of stellar populations, exoplanets, and primitive bodies in the solar system, those measurements help map out how disks shape star and planet formation.

These disks and their initial structures are seeded when a star is made. Star formation begins with the gravitational collapse of an over-dense core in a molecular cloud. An initial nudge that imparts some core rotation means that material collapsing from its outer regions (with higher angular momentum) is channeled onto a disk, rather than the protostar itself (Terebey et al. 1984). In that sense, disks are simple consequences of angular momentum 


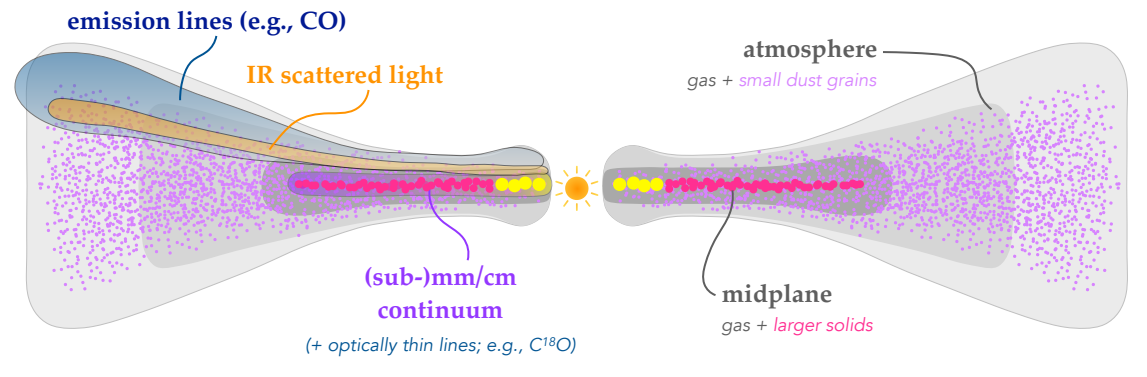

Figure 1

A cartoon schematic of a disk structure viewed in cross-section. The gas is denoted in grayscale, and solids are marked with exaggerated sizes and colors. The left side highlights the approximate locations of emission tracers; the right side defines some structure and contents terminology.

conservation. Measurements of young disk structures, still embedded in their natal core material, can reveal much about the star formation process: their sizes help distinguish the roles that magnetic fields have in regulating core collapse; their masses help constrain protostellar accretion rates; and their density distributions encode the angular momentum transport that ultimately determines the stellar mass (see the review by Li et al. 2014).

Disks are also the material reservoirs and birthplaces of planetary systems. The prevalence, formation modes, masses, orbital architectures, and compositions of planets depend intimately on the physical conditions in the disk at their formation sites, the evolution of that disk structure (locally and globally), and the planetary migration driven by dynamical interactions with the disk material. Measurements of the disk mass, its spatial distribution, and its demographic dependences offer crucial boundary conditions for models of planet formation. Combined with the properties observed in the mature exoplanet population, that information can help develop and refine a predictive formation theory, despite the considerable complexity of the associated physical processes (e.g., Benz et al. 2014).

\subsection{Observational Primer}

In these and many other ways, disk structures offer profound insights on how the properties of stars and planetary systems are shaped by their origins. This review is focused on the recent landscape of observational constraints on disk structures: how relevant measurements are made, what they suggest about disk properties, and how those properties are connected to star and planet formation. The most valuable measurements employ data with high angular resolution, as the typical nearby $(d \approx 150 \mathrm{pc})$ disk subtends $\lesssim 1^{\prime \prime}$ on the sky. Most of any given disk is cool enough $(<100 \mathrm{~K})$ that it emits efficiently at (sub-)mm wavelengths. Coupling these small angular sizes and cool temperatures, this review emphasizes radio interferometry as an essential tool. Indeed, progress over the past decade has largely been driven by the commissioning of the transformational ALMA facility.

Three categories of observational tracers are used to study disk structures: scattered light, thermal continuum emission, and (primarily molecular) spectral line emission. The first two are sensitive to the physical conditions and distribution of the solids, and the third is used to measure the properties of the gas. Figure 1 shows a schematic diagram that highlights the basic aspects of disk structure and the (two-dimensional) locations where 

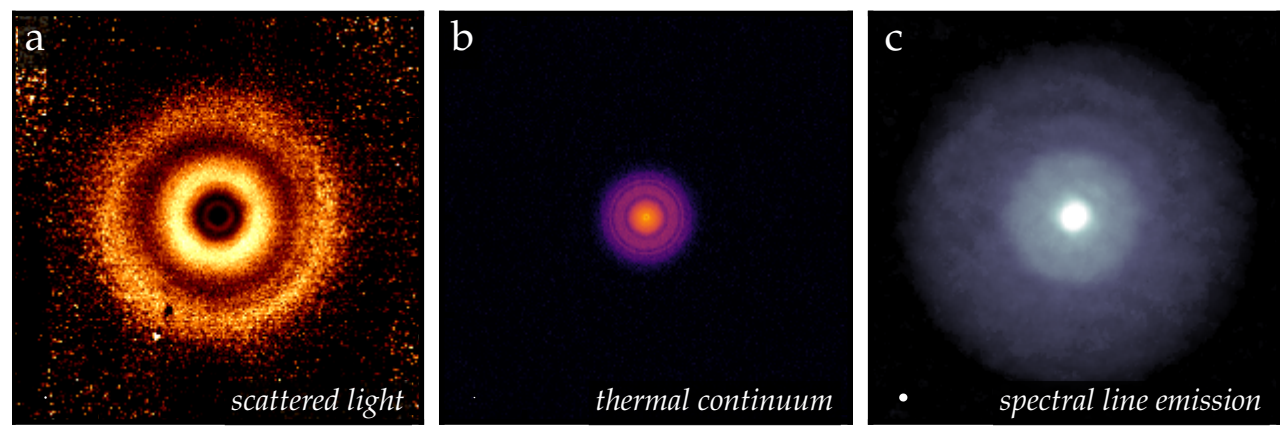

Figure 2

The morphology of the TW Hya disk is compared in three different tracers: (a) $\lambda=1.6 \mu \mathrm{m}$ scattered light from small dust grains (van Boekel et al. 2017), (b) $\lambda=0.9 \mathrm{~mm}$ continuum from pebble-sized particles (Andrews et al. 2016), and (c) the CO $J=3-2$ spectral line emission tracing the molecular gas (Huang et al. 2018a). Each panel spans 500 au on a side; resolutions are shown with ellipses in the lower left corner of each panel (too small to be visible in a and b). It is helpful to compare these emission distributions with the behavior in the Figure 1 schematic.

these tracers originate. Each of these probes is sensitive to different materials and physical conditions, ensuring considerable diversity in the disk appearance when viewed in different tracers. An illustrative example is shown in Figure 2.

1.2.1. Scattered Light. Small ( $\sim \mu \mathrm{m}$-sized) dust grains suspended in the gas at a suitable altitude in the disk atmosphere reflect the radiation emitted by the host star. This scattered light is sensitive to the radial variation of the vertical height of the dust distribution (Section 4.3). The spectral and polarization behavior of the scattered light constrain the albedos, set by the sizes, shapes, and compositions of the grains (Section 4.2). The practical advantage of this tracer is resolution: adaptive optics systems operating near the diffraction limit on 8-10 $\mathrm{m}$ telescopes measure features at 30-50 milliarcsecond scales ( $\sim 5$ au at the typical distances of nearby star-forming regions, $\sim 150 \mathrm{pc}$ ). The important challenges include: contrast with the host star, preventing measurements in the innermost disk $(\lesssim 10 \mathrm{au})$; sensitivity at large radii, due to the dilution of the stellar radiation field; and technical limits on the host star brightness. Taken together, those issues bias the current sample of resolved scattered light measurements toward disks with more massive hosts.

1.2.2. Continuum Emission. Disk solids emit a thermal continuum that spans four decades in wavelength $(\lambda \approx 1 \mu \mathrm{m}-1 \mathrm{~cm})$. Most of that emission is optically thick, and therefore a temperature diagnostic. Optical depths $\left(\tau_{\nu}\right)$ decrease with $\lambda$; the transition to $\tau_{\nu} \lesssim 1$ is traditionally expected in the sub-mm. In the optically thin limit, the intensity $\left(I_{\nu}\right)$ scales with the surface density of solids $\left(\Sigma_{\mathrm{s}}\right.$; Section $\left.2.1,2.3\right)$, and its spectral dependence is sensitive to the solid particle properties (Section 4.3). This tracer is bright, accessible at high resolution (to 10-20 milliarcseconds, or $\sim 2 \mathrm{au}$ ), and has no stellar contrast limitations. Accordingly, measurements are plentiful: much of the collective knowledge about disk structures is based on $\mathrm{mm}$ continuum data. The disadvantages arise from ambiguities in the detailed particle properties and the validity of the optically thin approximation (Sections 4 and 5). 
1.2.3. Spectral Line Emission. The most abundant molecule in a disk $\left(\mathrm{H}_{2}\right)$ does not have a permanent dipole moment and does not emit efficiently over the vast majority of the disk volume. The bulk of the gas in a disk is essentially 'dark', and there is no direct probe of its mass reservoir. Instead, measurements rely on the spectral line emission from (sub-)mm rotational transitions of rare tracer molecules. Optically thick line intensities are sensitive to the temperature in the atmospheric layer that corresponds to the line photosphere (Section 2.4). At low optical depths, line intensities are a function of both temperature and density. If the abundance of a given species relative to $\mathrm{H}_{2}$ (denoted here as $\mathrm{X}_{j}$ for molecule $j$ ) is known, spatially resolved maps of optically thin line emission constrain the gas surface density profile, $\Sigma_{\mathrm{g}}$ (Section 2.1, 2.3). Moreover, spectrally resolved line emission can be used to tomographically reconstruct the disk velocity field (Section 2.5).

ALMA is now capable of resolving emission lines at tens of milliarcsecond scales $(\sim 5$ $\mathrm{au}$ ) in velocity channels only a few $\mathrm{m} \mathrm{s}^{-1}$ wide, but the narrow bandwidths and low abundances of trace molecular species mean that sensitivity is a perennial challenge for disk measurements. Accordingly, line measurements of disks are much less common than for the continuum. The most prominent obstacles in interpreting spectral line data are high optical depths, confusion with the emitting layer height (when resolution is limited), and the large (potentially orders of magnitude) uncertainties in the molecular abundances $\left(\mathbf{X}_{j}\right)$.

\subsection{Statement of Scope}

Keeping in mind the motivations for measuring disk structures and the observational tools that are now available, this review covers four broad (and inter-related) topics that occupy much of the effort in the disk research community: inferred physical characteristics of disk structures and their ambiguities (Section 2); empirical constraints on evolutionary and environmental dependencies based on demographics studies (Section 3); evidence for (and problems with) the growth and migration of disk solids (Section 4); and the properties and roles of small-scale substructures in shaping observables and facilitating planet formation (Section 5). The review concludes with a brief synopsis that summarizes the current state of the field and some suggestions of potentially fruitful avenues for future work (Section 6).

\section{KEY STRUCTURE PROPERTIES}

The spatial distribution of mass - the density structure - is without question the fundamental property of interest for disks. The conceptual orientation of the entire field presumes that disk evolution is deterministic: in principle, a collection of density structure measurements that span an appropriate range of environmental and evolutionary states could be used to work out the mechanics of key evolutionary processes. This section of the review is focused on the underlying motivations, observational constraints, and lingering ambiguities associated with the mass distributions in disks (Sections 2.1-2.3). The intrinsic connections (physical and observational) between the density structure and the thermal and dynamical state of the disk material are summarized in Sections 2.4 and 2.5, respectively.

\subsection{Mass}

With a limited number of resolved disk measurements, more emphasis is placed on masses than density distributions. Nevertheless, the key issues can be illustrated from this coarser perspective. Masses offer elementary constraints on the future contents of planetary sys- 


\section{Notation, Conventions, and Nomenclature}

To simplify discussions, variable notations are used throughout this review. Cylindrical coordinates define the disk frame of reference for these properties, where $(r, \theta, z)$ correspond to the radial distance from the star, the azimuthal angle around the disk, and the height above the disk midplane, respectively. Many structural and observational properties vary in three dimensions. To minimize complexity and avoid confusion, the convention is to explicitly note spatial dependencies only when the spatial behavior is directly relevant (e.g., most discussion presumes azimuthal symmetry). For example: $T$ is shorthand for the local value $T(r, \theta, z)$; $T(r)$ refers to a radial profile at a given (e.g., the midplane) or generic $z$, depending on the context; and $\langle T\rangle$ refers to a disk-averaged quantity, $\langle T\rangle=\iiint T(r, \theta, z) r d r d \theta d z / \iiint r d r d \theta d z$.

tems. The current census of exoplanets finds an abundance of worlds orbiting other stars, but the metamorphosis of disk material into planetary systems is unclear without a comparison of the available mass reservoirs in the parent and descendant populations. Summing the masses of terrestrial planets and giant planet cores in the solar system, or an ensemble of exoplanets, offers a conservative lower bound on the solid mass expected in their progenitor disks, $M_{\mathrm{s}} \gtrsim 40 \mathrm{M}_{\oplus}$ (Weidenschilling 1977a; Chiang \& Laughlin 2013). Extrapolations of the current planetary atmosphere compositions to the primordial gas expected in disks give an analogous bound for the gas masses, $M_{\mathrm{g}} \gtrsim 3000 \mathrm{M}_{\oplus}$.

Solids are a minor contributor to the mass budget, with an initial mass fraction of $\sim 1 \%$ relative to the gas. But the fundamental roles they play in all aspects of disk evolution and planet formation justify special attention to their mass reservoir. The optimal $M_{\mathrm{s}}$ diagnostic is the luminosity of the $\mathrm{mm}$ continuum emission, $L_{\mathrm{mm}}$. In the optically thin limit, the continuum intensity scales like $I_{\nu} \propto \kappa_{\nu} B_{\nu}(T) \Sigma_{\mathrm{s}}$, where $\kappa_{\nu}$ is the absorption opacity, $B_{\nu}(T)$ the Planck function at temperature $T$, and $\Sigma_{\mathrm{s}}$ the surface density of solids. Integrating that emission over the disk volume shows that $L_{\mathrm{mm}} \propto M_{\mathrm{s}}$. Figure 3 shows the $M_{\mathrm{s}}$ distribution inferred from mm continuum photometry surveys for 887 disks. Though that distribution is subject to considerable ambiguities (see below) and biased by observational and physical selection effects (Section 3), it offers rough guidance on typical $M_{\mathrm{s}}$ values.

Estimates of $M_{\mathrm{s}}$ are intrinsically uncertain because they rely on assumptions about the properties of the emitting particles. A detailed discussion of those properties, encapsulated in the absorption opacity, is deferred to Section 4, but the standard approach is to adopt a reasonable estimate that maximizes $\left\langle\kappa_{\nu}\right\rangle$. Coupled with the possibility that some of the continuum emission is optically thick (Beckwith et al. 1990; Andrews \& Williams 2005; Zhu et al. 2019), this implies that $M_{\mathrm{s}}$ estimates are more appropriately considered lower bounds. The sense of that ambiguity factors into comparisons between the distributions of disk and planetary system masses, as highlighted in the box at the end of this section.

There are many fewer estimates of $M_{\mathrm{g}}$, primarily because $\mathrm{mm}$ spectral line observations are more expensive than for the continuum. One option for a mass-sensitive tracer molecule is HD, the primary isotopologue of $\mathrm{H}_{2}$ (Bergin et al. 2013; McClure et al. 2016). The advantage of HD is the simplicity of its associated chemical network, which builds confidence in estimates of its abundance, $\mathrm{X}_{\mathrm{HD}}$. But with a ground state transition at 112 $\mu \mathrm{m}, \mathrm{HD}$ measurements are scarce (three disk detections) and currently inaccessible (with no operational far-infrared space telescope). Estimates of $M_{\mathrm{g}}$ based on HD have a strong 


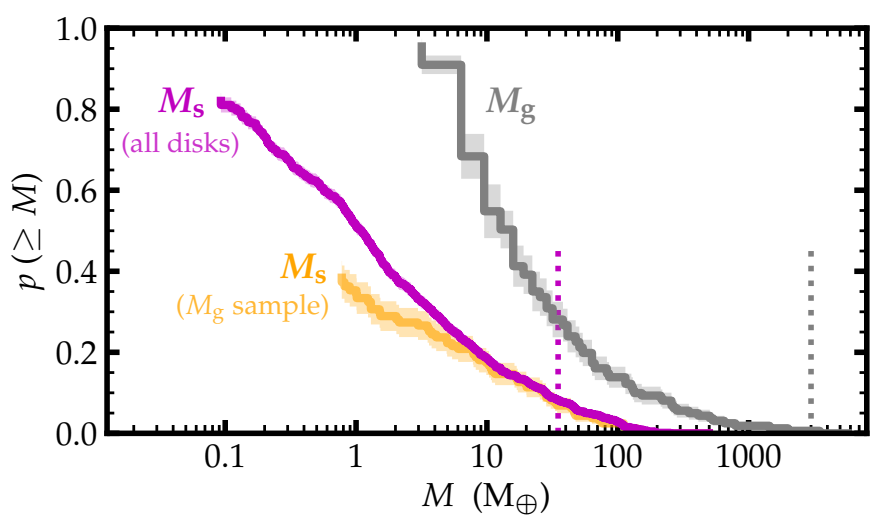

Figure 3

Cumulative distributions of disk solid $\left(M_{\mathrm{S}}\right)$ and gas $\left(M_{\mathrm{g}}\right)$ masses. The $M_{\mathrm{S}}$ distribution (purple) includes 887 disks in Oph (Cieza et al. 2019; Williams et al. 2019), Tau (Andrews et al. 2013; Akeson et al. 2019), Lup (Ansdell et al. 2016, 2018), Cha (Pascucci et al. 2016; Long et al. 2018a),

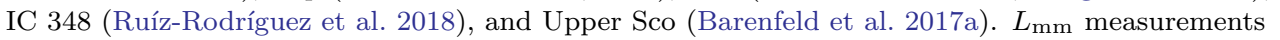
at $\lambda=0.9 \mathrm{~mm}$ (or $1.3 \mathrm{~mm}$, scaled by $\nu^{2.2}$; Section 4.3.2) were converted to $M_{\mathrm{s}}$ assuming $\langle T\rangle=20$ $\mathrm{K}$ and $\left\langle\kappa_{\nu}\right\rangle=3.5 \mathrm{~cm}^{2} \mathrm{~g}^{-1}$. The $M_{\mathrm{g}}$ distribution (gray) uses $\mathrm{CO}$ isotopologue data (Ansdell et al. 2018; Long et al. 2018a; with a small supplement from individual case studies) and employs the models of Miotello et al. (2017). Since such line data are rare and the sample biased, the $M_{\mathrm{s}}$ distribution for the same disks is also shown (orange) for comparison. The minimum masses in solids and gas needed to produce the solar system planets are shown as dashed vertical lines in purple and gray, respectively (following Weidenschilling 1977a).

$T$-dependence and are considered lower bounds for two reasons. First, there are potential alternative reaction pathways (e.g., into hydrocarbons) that could lower $\mathrm{X}_{\mathrm{HD}}$. And second, the line may be optically thick, and some of the emission could be hidden below the optically thick local continuum. These latter issues can be treated by comparing the data with radiative transfer models that interpret a prescription for the two-dimensional temperature and density structures (e.g., McClure et al. 2016; Trapman et al. 2017).

$\mathrm{CO}$ is a more common gas tracer in disks, since the abundance is high and the lowenergy rotational transitions are easily accessed with $\mathrm{mm}$ interferometers. The primary isotopologue has very high optical depths (Beckwith \& Sargent 1993), so $M_{\mathrm{g}}$ estimates rely instead on rarer species (usually ${ }^{13} \mathrm{CO}$ and $\mathrm{C}^{18} \mathrm{O}$ together) and references to parametric model catalogs (Williams \& Best 2014; Miotello et al. 2016). A modest (and biased) collection of $M_{\mathrm{g}}$ measurements are available from assorted case studies and shallow line surveys (e.g., Ansdell et al. 2016, 2018; Long et al. 2017a), as shown in Figure 3.

These CO-based masses appear low, $\sim 5-10 \times$ lower than crude estimates from the product of the accretion rate and stellar age (Manara et al. 2016), or different gas tracers in the same disks (Favre et al. 2013; Kama et al. 2016b), or if $M_{\mathrm{s}}$ is scaled up by a standard gas-to-solids fraction (100; Dutrey et al. 2003; Ansdell et al. 2016). The anomaly can be reconciled with a lower gas-to-solids ratio $(\lesssim 10)$ or by decreasing $\mathrm{X}_{\mathrm{CO}}$ or the isotope fractionation below ISM values. Such abundance changes are expected from various processes (e.g., Miotello et al. 2017), including adsorption onto solids (Aikawa et al. 1997; van Zadelhoff et al. 2001), isotope-selective photodissociation (Miotello et al. 2014, 2016), and especially the sequestration of $\mathrm{C}$ or $\mathrm{O}$ into grains, ices, or other species (e.g., organ- 


\section{Is there enough mass in disks to make planetary systems?}

The mass distributions in Figure 3 suggest that few disks have enough material to produce the solar system or its counterparts in the exoplanet population. Interpretations of this discrepancy have been considered in various forms (Greaves \& Rice 2010; Najita \& Kenyon 2014; Manara et al. 2018), with proposed solutions falling into two (not mutually exclusive) categories. The first explanation is perhaps pessimistic, but it simply recalls that $M_{\mathrm{s}}$ and $M_{\mathrm{g}}$ estimates are lower bounds: biased accounting factors $\left(\kappa_{\nu}\right.$ or $\left.\mathrm{X}_{j}\right)$ and optically thick contamination could make the true masses much higher. For example, if the mm continuum emission used to estimate $M_{\mathrm{s}}$ includes contributions from $10 \mathrm{~cm}$ rocks instead of $1 \mathrm{~mm}$ pebbles, the $M_{\mathrm{s}}$ distribution would shift up an order of magnitude (Section 4.2). The second solution strikes a more optimistic tone, proposing instead that planet formation has already occurred and the observations are tracing the "leftovers" (e.g., collisional debris) rather than the actual mass. The concept of a condensed planet formation timescale, presumably occurring during the embedded phase (e.g., Nixon et al. 2018), has gained recent momentum from the fine-scale features that are now routinely identified in high resolution disk images (Section 5).

ISM: interstellar medium. ics; Reboussin et al. 2015; Yu et al. 2016, 2017; Miotello et al. 2017; Bosman et al. 2018) Alternatively, the typical $\mathrm{CO}$ isotopologue tracers (even $\mathrm{C}^{18} \mathrm{O}$ ) might be optically thick, saturating the line luminosities (Booth et al. 2019). The salient point is again that the standard adopted assumptions produce lower bounds on $M_{\mathrm{g}}$ by design.

\subsection{Size}

Sizes are a natural step in the progression of measurements from masses to density profiles. There is no consensus size definition, physically or observationally, since any metric depends on the adopted prescription for the radial variations of densities or intensities. A physical modeling effort to homogenize size measurements is littered with ambiguities. A more practical approach is to assign an empirical definition of an effective size, $R_{j}$, defined as the radius that encircles a fixed fraction of the luminosity from tracer $j$.

Resolved mm continuum measurements from roughly 200 disks have been used to infer $R_{\mathrm{mm}} \approx 10-500 \mathrm{au}$ (defined here so $R_{\mathrm{mm}}$ encircles $0.9 L_{\mathrm{mm}}$; Tripathi et al. 2017; Andrews et al. 2018a; Hendler et al. 2020). The lower bound of that range is presumably limited by resolution. Figure $4 \mathbf{a}$ shows a tight correlation between the $\mathrm{mm}$ continuum sizes and luminosities (Andrews et al. 2010; Piétu et al. 2014) with a scaling relation $L_{\mathrm{mm}} \propto R_{\mathrm{mm}}^{2}$ (Tripathi et al. 2017; Andrews et al. 2018a) that may flatten for older systems (Hendler et al. 2020). The origins of this relationship are not clear: it could be imposed at the disk formation epoch (Isella et al. 2009; Andrews et al. 2010), produced by the evolution of solids (Tripathi et al. 2017; Rosotti et al. 2019a; see Section 4), or it may be a more trivial manifestation of high optical depths (Andrews et al. 2018a; Zhu et al. 2019).

Scattered light images offer an alternative size metric for the solids, although the empirical methodology outlined above has not been used for such data in the literature. Nevertheless, the current suite of scattered light images (e.g., Garufi et al. 2018) demonstrate that the $\mu \mathrm{m}$-sized dust grains that reflect starlight are distributed out to greater distances than the larger particles responsible for the mm continuum (e.g., Figure 2). Quantifying this size difference can be especially difficult, in large part due to the dilution of the stellar 

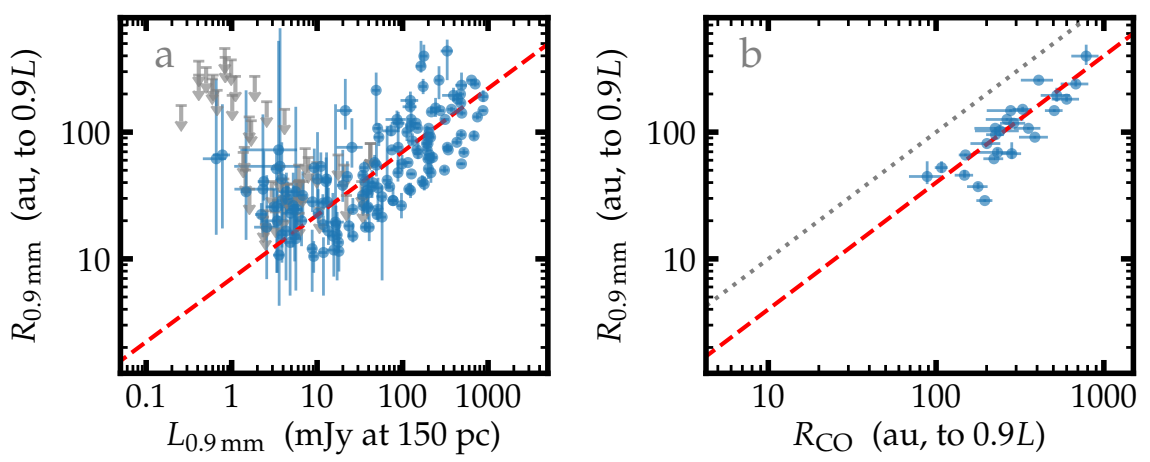

Figure 4

(a) The correlation between the $\lambda=0.9 \mathrm{~mm}$ continuum luminosities (in flux density units, scaled to a common distance of $150 \mathrm{pc}$ ) and sizes (the radii that encircle $0.9 \mathrm{~L}_{\mathrm{mm}}$; Tripathi et al. 2017; Andrews et al. 2018a; Hendler et al. 2020). The inferred scaling relation, $R_{\mathrm{eff}} \propto L_{\mathrm{mm}}^{0.5}$, is overlaid in red. (b) A comparison of $R_{\mathrm{mm}}$ and analogous sizes inferred from the CO line emission (data from Öberg et al. 2011; Simon et al. 2017; Ansdell et al. 2018; Facchini et al. 2019). A one-to-one marker is shown as a dotted line, and a $R_{\mathrm{CO}} \approx 2.5 R_{\mathrm{mm}}$ relation is shown in red.

radiation field in the outer disk, but future comparisons would be valuable.

Again, there are many fewer size measurements for the gas phase. The CO line emission extends to $R_{\mathrm{CO}} \approx 100-500$ au (Ansdell et al. 2018), although a few outliers stretch beyond the high end of that range. Smaller disks presumably exist, but produce such weak line emission that they are missing in current samples (e.g., Barenfeld et al. 2017b). The available data suggest $R_{\mathrm{CO}} \gtrsim 2 R_{\mathrm{mm}}$, as shown in Figure $4 \mathbf{b}$. Some of that difference is related to comparing tracers with such different optical depths (Hughes et al. 2008; Trapman et al. 2019), but radiative transfer models argue for a genuine discrepancy between the density distributions (Panić et al. 2009; Andrews et al. 2012; Facchini et al. 2019).

There is a significant caveat in these measurements and results that merits reiteration: these are empirical size measurements that are not directly or simply linked to the density distribution. While the inferred behaviors outlined above may point to fundamental physical relationships, a translation into physical radii is not obvious (Rosotti et al. 2019b).

\subsection{Density}

In principle, spatially resolved measurements of the mass tracers introduced in Section 2.1 can constrain the surface density profiles for the solids $\left(\Sigma_{\mathrm{s}}\right)$ or gas $\left(\Sigma_{\mathrm{g}}\right)$. Measurements of $\Sigma_{\mathrm{g}}$ offer important insights on how angular momentum is transported in disks through turbulent viscosity (Hartmann et al. 1998) or winds (Blandford \& Payne 1982; Bai \& Stone 2013), and what types of planetary system architectures can be formed (Miguel et al. 2011) and how they will evolve via migration (Baruteau et al. 2014). Likewise, the evolution of $\Sigma_{\mathrm{s}}$ is a diagnostic of the processes that drive the growth of dust grains into planetesimals (Johansen et al. 2009; Birnstiel et al. 2012). Put simply, the disk density structure ties into all the fundamental physical mechanisms relevant to star and planet formation.

A key emphasis has been on estimating $\Sigma_{\mathrm{s}}$ from modest resolution $(\sim 20-50 \mathrm{au})$ observations of mm continuum morphologies (Andrews \& Williams 2007a; Piétu et al. 2007, 2014; Andrews et al. 2009, 2010; Isella et al. 2009; Tazzari et al. 2017). The modeling details used 
to make those measurements vary substantially between studies, but a crude distillation suggests $\Sigma_{\mathrm{s}} \propto r^{-1}$ or shallower in the inner disk and $\Sigma_{\mathrm{s}} \propto r^{-3}$ or steeper at large $r$. At these resolutions, the density gradient is actually measured at large $r$ (tens to hundreds of $\mathrm{au}$ ); estimates of $\Sigma_{\mathrm{s}}$ in the inner disk are extrapolated according to the prescribed functional form of the density profile. At $r \approx 50-100 \mathrm{au}, \Sigma_{\mathrm{s}}$ values span $\sim 0.001-1 \mathrm{~g} \mathrm{~cm}^{-2}$.

The basic methodology for inferring $\Sigma_{\mathrm{g}}$ from spectral line observations is similar (e.g., Williams \& McPartland 2016; Zhang et al. 2019). The focus has been almost exclusively on the CO isotopologues as the density tracers (e.g., Miotello et al. 2018; there is no prospect for spatially resolved HD measurements). Some of the intrinsic degeneracies can be mitigated by modeling multiple species or line transitions simultaneously (van Zadelhoff et al. 2001; Dartois et al. 2003; Schwarz et al. 2016; Zhang et al. 2017; Cleeves et al. 2017).

Density measurements suffer the same ambiguities outlined for the masses (Section 2.1); namely, the uncertain conversion factors $-\kappa_{\nu}$ for $\Sigma_{\mathrm{s}}$ and $\mathrm{X}_{\mathrm{CO}}$ (and isotopic fractions) for $\Sigma_{\mathrm{g}}$ - and the potential for contamination by high optical depths. There is also the added complexity that high $\Sigma_{\mathrm{s}}$ could produce an optically thick continuum that blocks the spectral line emission originating below (or behind) the continuum photosphere (e.g., Weaver et al. 2018; Isella et al. 2018). If that effect is significant, robust estimates of $\Sigma_{\mathrm{g}}$ from line data will also require a simultaneous inference of $\Sigma_{\mathrm{s}}$ (a formidable challenge).

Measurements of disk densities remain in an exploratory phase, with progress limited by data availability and quality, systematics in the methodology, and intrinsic degeneracies. Some promising ideas for measuring $\Sigma_{\mathrm{g}}$ aim to get around the tracer abundance ambiguity, using line ratios that are directly sensitive to the volume density (e.g., Teague et al. 2018 b) or converting multiwavelength $R_{\mathrm{mm}}$ measurements and a simplified model for the aerodynamics of solids to an inference of the underlying density profile (Powell et al. 2017).

\subsection{Temperature}

The thermal structure determines some fundamental reference scales, usually parameterized by the sound speed, $c_{\mathrm{s}}\left(\propto T^{0.5}\right)$, and the pressure scale height, $H_{p}=c_{s} / \Omega_{\mathrm{k}} \propto\left(\operatorname{Tr}^{3} / M_{*}\right)^{0.5}$, where $\Omega_{\mathrm{k}}$ is the Keplerian angular velocity. Moreover, it is intimately connected to the tracers of the disk material, since it controls the molecular excitation conditions, the vertical location of the scattering surface, and the spectral line and continuum intensities.

The temperature distribution depends on the irradiation of solids by the host star. Small grains suspended in the disk atmosphere absorb starlight and then re-radiate some of that energy toward the midplane (Chiang \& Goldreich 1997; D'Alessio et al. 1998). That central, external energy deposition produces an increasing $T(z)$ (Calvet et al. 1991) and a decreasing $T(r)$ (Kenyon \& Hartmann 1987). Irradiation heating depends on the host star spectrum as well as the microphysical properties and vertical distribution of the solids (D'Alessio et al. 1999, 2006; Dullemond et al. 2001, 2002). The latter is set by a balance between turbulent mixing and the solids-gas coupling (Dubrulle et al. 1995). When the solids-to-gas ratio is low (at large $z$ or $r$ ), spectral line processes can super-heat the gas (Kamp \& Dullemond 2004; Bruderer 2013). A variety of secondary heating sources - viscous dissipation (D'Alessio et al. 1998), spiral shocks (Rafikov 2016), radioactivity (Cleeves et al. 2013), external irradiation (e.g., from an envelope; Natta 1993; D'Alessio et al. 1997), or vertical structure perturbations (e.g., from self-shadowing; Dullemond \& Dominik 2004b) - can also contribute significantly to the temperature structure.

The classical approach to constraining the temperature distribution is to forward-model 


\section{Observational Insights on Disk Magnetic Fields}

Magnetic fields are predicted to fundamentally alter the gas dynamics in disks, and thereby play important roles in shaping their structures and evolution. But there are few concrete observational constraints available to inform magnetohydrodynamics models. In principle, magnetic field morphologies can be measured from the linear polarization of mm continuum emission (Cho \& Lazarian 2007; Bertrang et al. 2017) or molecular line emission (Goldreich \& Kylafis 1981). So far, efforts to measure the former have been frustrated by scattering (Kataoka et al. 2015) and various alternative grain alignment mechanisms that can also polarize the continuum (e.g., Tazaki et al. 2017; Kataoka et al. 2019). Linear polarization measurements of spectral lines from disks are expected soon. The line-of-sight magnetic field strength (and topology) can potentially be measured with high resolution spectral line observations of circular polarization induced by Zeeman splitting (e.g., in CN hyperfine transitions; Brauer et al. 2017).

the infrared SED. Such modeling proposes a density and opacity distribution, simulates the propagation of energy through the disk, generates synthetic observables to compare with data, and iterates. The fundamental challenges are the physical degeneracies in such modeling (Thamm et al. 1994; Heese et al. 2017); even if internally self-consistent, the models are not unique. One way to mitigate some ambiguity is to fold additional (spatially resolved) data into the modeling circuit (e.g., Pinte et al. 2008).

Another option relies on the spatial distribution of optically thick emission lines (Weaver et al. 2018). With sufficient resolution, $T(r)$ can be measured in the vertical layer corresponding to the line photosphere (Rosenfeld et al. 2013a; Pinte et al. 2018a; Dullemond et al. 2020). Constraints on $T(r, z)$ are possible by probing intensities at different depths in the atmosphere using lines with a range of excitation conditions (van Zadelhoff et al. 2001; Dartois et al. 2003; Schwarz et al. 2016). That reconstruction effort can be supplemented with benchmarks in $T(r)$ from signposts of condensation fronts (snowlines), where volatiles are removed from the gas when they freeze onto grain surfaces (Qi et al. 2011, 2019).

\subsection{Dynamics}

Disks are profoundly affected by their fluid dynamics (Armitage 2011). The dominant factor in the kinematic structure of a disk is orbital motion, but important contributions are expected from magnetic fields (Turner et al. 2014; see the box above), viscous transport (Lynden-Bell \& Pringle 1974), pressure support (Weidenschilling 1977b), self-gravity (Rosenfeld et al. 2013a), and winds (Ercolano \& Pascucci 2017). Random motions generated by turbulence are traditionally asserted as the source of a kinematic viscosity $\left(\nu_{\mathrm{t}}\right)-$ quantified with the coefficient $\alpha_{\mathrm{t}}=\nu_{\mathrm{t}} / c_{\mathrm{s}} H_{p}$ - that controls accretion, mixing, and other diffusive processes. Classical models of turbulence driven by the MRI (Balbus \& Hawley 1991 ) predict $\alpha_{\mathrm{t}} \approx 0.001-0.01$. But a shifting theoretical paradigm now argues that the MRI is suppressed by non-ideal MHD effects over much of the disk (e.g., Bai \& Stone 2013), suggesting instead a system that is effectively laminar, $\alpha_{\mathrm{t}}<0.001$.

Spatially and spectrally resolved observations of emission lines with a range of optical depths can be used to reconstruct the three-dimensional disk velocity field. Typical observations are suitable for confirming that orbital motions dominate (Rosenfeld et al.
SED: spectral energy distribution.

MRI: magnetorotational instability.

MHD: magnetohydrodynamics. 
2012a; Czekala et al. 2015; Simon et al. 2017), although measurements of non-Keplerian deviations are becoming available (Section 5). Constraints on turbulence are available from two approaches. The first relies on a measurement of spectral broadening: an emission line profile has contributions from both thermal and non-thermal motions, with characteristic variances $2 k_{\mathrm{B}} T / m_{j}$ (where $k_{\mathrm{B}}$ is the Boltzmann constant and $m_{j}$ the mass of molecule $j$ ) and $\delta v_{\mathrm{t}}^{2}$, respectively. With some knowledge of $T(r, z)$ (usually inferred jointly), resolved line measurements constrain $\delta v_{\mathrm{t}}$ in a given line photosphere layer. Suitable data are only available in three cases. In two of these (TW Hya and HD 163296), upper limits indicate sub-sonic turbulence $\left(\delta v_{\mathrm{t}} \lesssim 0.05 c_{\mathrm{s}}\right)$ at $z \approx 1-3 H_{p}$, corresponding to $\alpha_{\mathrm{t}} \lesssim 0.005$ (Hughes et al. 2011; Flaherty et al. 2015, 2017, 2018; Teague et al. 2016, 2018c). A much broader $\delta v_{\mathrm{t}}\left(\sim 0.5 c_{\mathrm{s}}\right)$ is found in the remaining case (DM Tau; Guilloteau et al. 2012). Taken at face value, this implies vigorous turbulence $\left(\alpha_{\mathrm{t}} \gtrsim 0.1\right)$ at a comparable altitude, or it may hint that the $T$ distribution is incorrect or other broadening mechanisms are at play.

The second approach relies on the diffusive blurring of nominally "sharp" features (Section 5). High resolution mm continuum observations offer geometric constraints on turbulent mixing, based on the height of the mm photosphere (Pinte et al. 2016) or the radial widths of narrow ring features (Dullemond et al. 2018), that suggest $\alpha_{\mathrm{t}} \lesssim 10^{-3}$ near the midplane. This methodology is complementary to the line broadening, with each approach probing different altitudes with orthogonal degeneracies (gas-particle coupling and the thermal structure of the gas, respectively). Efforts to combine them can construct a more nuanced view of the spatial variation and origins of disk turbulence (e.g., Shi \& Chiang 2014).

\section{DEMOGRAPHIC INSIGHTS}

The previous section highlighted the design and vetting of tools used to infer physical aspects of disk structures, as well as the intrinsic ambiguities and practical limitations that frustrate those inferences. Those challenges are being confronted, with improved physical constraints following in step with the quality, volume, and diversity of the available data. But assembling large, homogeneous catalogs of robust disk structure models is simply not practical. Recognizing that, one imperative message from Section 2 is that theoretical work in the physical domain ultimately needs to transform outputs into appropriate observational metrics: predictions and model tests should happen in the data-space.

A proper demographic analysis requires a catalog of a given disk property (dependent variable) that is both large and spans a sufficient range in the external factors (independent variables) of interest. The two empirical probes of disk structure properties that are simple enough to measure in large quantities today are the mm continuum luminosities $\left(L_{\mathrm{mm}}\right)$ and sizes $\left(R_{\mathrm{mm}}\right)$. The remainder of this section synthesizes various data repositories to explore how these structure proxies depend on host masses (Section 3.1), the local and global environments (Section 3.2), and evolutionary diagnostics (Section 3.3).

\subsection{Links to Stellar Hosts}

Considerable attention in the field is devoted to probing connections between disk structures and their stellar hosts. In particular, most theoretical work associated with star and planet formation presumes that fundamental physical principles like the conservation of mass and angular momentum could imprint some lasting relationships between the stellar host masses, $M_{*}$, and basic disk structure metrics like masses and sizes. Some credence is lent to that 

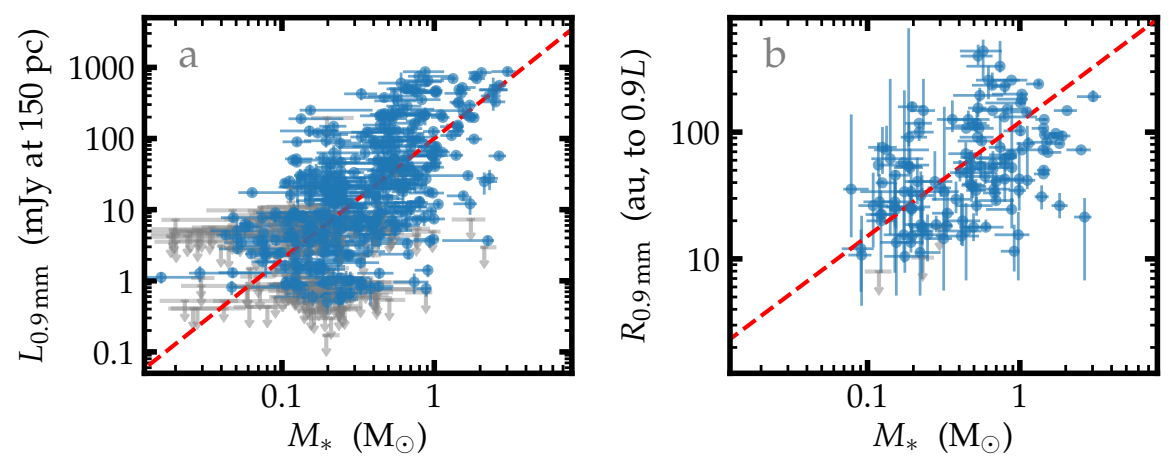

Figure 5

(a) The correlation between $L_{\mathrm{mm}}$ and $M_{*}$ for disks (excluding multiple systems) in the Oph, Tau, Lup, Cha I, IC 348, and Upper Sco regions (see references and details on calculations in Figure 3). Upper limits are shown as gray arrows. The red line corresponds to the mean scaling relation inferred for the Oph, Tau, Lup, and Cha I disks only. (b) The correlation between $R_{\text {mm }}$ (defined as in Figure 4) and $M_{*}$, along with the mean scaling relation shown in red.

emphasis from the clear $M_{*}$-dependencies that have been identified through demographic studies of the exoplanet population (e.g., see Mulders 2018).

Large mm continuum photometry catalogs for disks in a few nearby regions have sufficient dynamic range in $M_{*}$ to characterize any relationships with $L_{\mathrm{mm}}$ (Andrews et al. 2013; Ansdell et al. 2016; Pascucci et al. 2016; Barenfeld et al. 2017a). When excluding known multiple star systems (Section 3.2), the regions with mean ages $\lesssim 3 \mathrm{Myr}$ exhibit a consistent scaling relation, $L_{\mathrm{mm}} \propto M_{*}^{1.7 \pm 0.3}$ (for $M_{*} \geq 0.1 \mathrm{M}_{\odot}$; the same scaling is found at $\lambda=0.9$ or $1.3 \mathrm{~mm}$ ), shown in Figure $5 \mathbf{a}$. The normalization indicates that a typical disk with a solar analogue $\left(M_{*}=1 \mathrm{M}_{\odot}\right)$ host has flux densities of $\sim 100$ or $40 \mathrm{mJy}$ at $150 \mathrm{pc}$ for $\lambda=0.9$ or $1.3 \mathrm{~mm}$, respectively. There is considerable scatter around the mean $L_{\mathrm{mm}}-M_{*}$ relation, roughly a factor of three $(0.5 \mathrm{dex})$ added dispersion in $L_{\mathrm{mm}}$ beyond the measurement uncertainties. Some of that could be related to imprecise (or biased) $M_{*}$ estimates, though various physical origins are plausible. There is a hint for Taurus disks (Ward-Duong et al. 2018; Akeson et al. 2019) that extending to $M_{*}<0.1 \mathrm{M}_{\odot}$ flattens the mean relation $\left(L_{\mathrm{mm}} \propto M_{*}^{1.2}\right)$. It is unclear if this is a real turnover or if it is unique to Taurus.

Assuming the emission is optically thin, this relation predicts a corresponding scaling between $M_{\mathrm{s}}$ and $M_{*}$ with a morphology that is sensitive to the behavior of the disk-averaged temperatures and opacities. To date, all studies have presumed that $\left\langle\kappa_{\nu}\right\rangle$ is unrelated to $M_{*}$ (although without justification). Various treatments of the $M_{*}$-dependence on $\langle T\rangle$ have been considered: Andrews et al. (2013) suggested that $\langle T\rangle \propto L_{*}^{1 / 4} \propto M_{*}^{1 / 2}$ based on simple irradiation heating arguments, while Pascucci et al. (2016) preferred the assumption that $\langle T\rangle$ is independent of $M_{*}$. Given the measured behaviors of the size-luminosity (Section 2.2; Tripathi et al. 2017) and size $-M_{*}$ (Figure 5b; Andrews et al. 2018a) relations, simple irradiation heating should impose only a weak mass dependence on $\langle T\rangle$ (see Tazzari et al. 2017), in line with the Pascucci et al. assumption and therefore predicting a steeper than linear $M_{\mathrm{s}}-M_{*}$ relation (i.e., a nearly $M_{*}$-independent link between $L_{\mathrm{mm}}$ and $M_{\mathrm{s}}$ ).

The $R_{\mathrm{mm}}-M_{*}$ relation in Figure $5 \mathbf{b}$ is less pronounced, partly because the dynamic range in $M_{*}$ is limited (relative to the scatter) by resolution. Andrews et al. (2018a) 

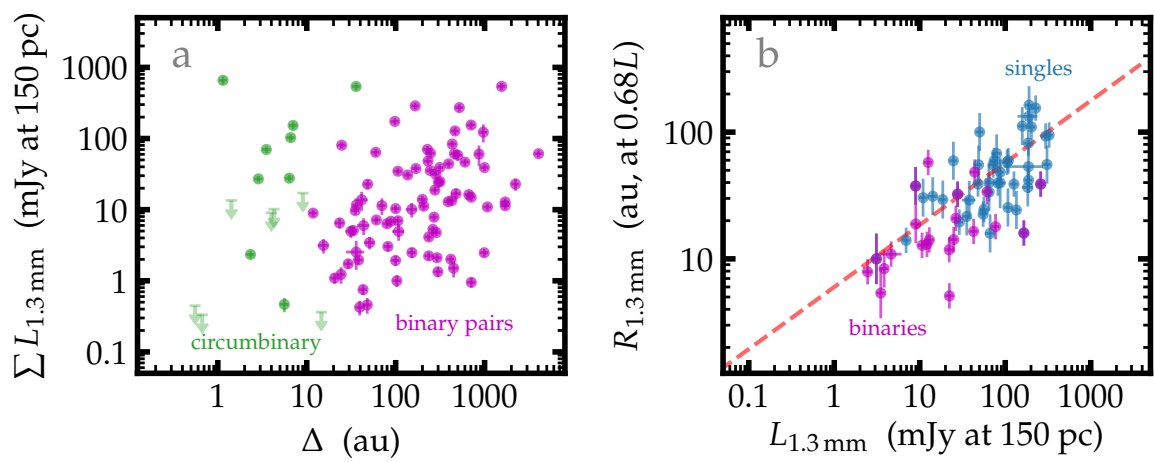

Figure 6

(a) The summed continuum luminosities $(\lambda=1.3 \mathrm{~mm})$ for binary pairs as a function of their projected separation. Known circumbinary disks are marked in green. The $L_{\mathrm{mm}}-\Delta$ behavior is qualitatively consistent with predictions of tidal truncation models. (b) The $\lambda=1.3 \mathrm{~mm}$

$R_{\mathrm{mm}}-L_{\mathrm{mm}}$ relation (in this case, $R_{\mathrm{mm}}$ is defined as the radius encircling $68 \%$ of $L_{\mathrm{mm}}$ ) for disks in binary systems (blue; Manara et al. 2019), compared with disks around similar, but single, hosts (orange; Andrews et al. 2018b; Long et al. 2019). The red line marks the $\lambda=0.9$ mm relation (Section 2.2), renormalized with $L_{\mathrm{mm}} \propto \nu^{2.2}$ and $R_{\mathrm{mm}} \propto \nu^{0.3}$ (Section 4).

estimated that a slightly sub-linear relationship was appropriate: the updated results here suggest $R_{\mathrm{mm}} \propto M_{*}^{0.9}$, consistent with a simple combination of the measured $L_{\mathrm{mm}}-M_{*}$ and $L_{\mathrm{mm}}-R_{\mathrm{mm}}$ scaling relations. If the emission is optically thin, Andrews et al. (2018a) demonstrated that such scaling behavior naturally follows if all disks have a similar mm optical depth profile (independent of $M_{*}$ ) with $\left\langle\tau_{\nu}\right\rangle \approx 0.4$, meaning $M_{\mathrm{s}}$ depends primarily on the disk size. Alternatively, the same relationships would be produced if the emission is optically thick with an effective filling factor of $\sim 0.3$, produced by spatially concentrating the high optical depths (Ricci et al. 2012) or reducing the intensities by self-scattering from particles with high albedos (Zhu et al. 2019). The scatter in these relations can be attributed to diversity in the underlying relation between the disk sizes and host masses, the mean optical depths, effective filling factors, or a combination of such effects.

\subsection{Environmental Effects}

3.2.1. Dynamical Interactions. Disk structures can be substantially shaped by dynamical interactions in their local environments. The tidal perturbations that occur in multiple star systems are expected to be the most prevalent for the current catalog of disk observations (although see the box on the next page). Multiplicity fractions are high, 30-50\% in the field (Raghavan et al. 2010) and up to $\sim 70 \%$ for the young clusters that inform most disk studies (e.g., Kraus et al. 2011). Moreover, most stellar pairs have separations comparable to typical disk sizes $(\sim 10-100 \mathrm{au})$. Simulations of the perturbations to disk structures induced by gravitational interactions in such systems find that individual disks in binaries are tidally truncated at $r \approx(0.2-0.5) \Delta$, where $\Delta$ is their mean separation (Artymowicz \& Lubow 1994); they generically predict that disks in close binaries are smaller, and therefore less massive, than their counterparts in wider binaries or around single stars.

There is some qualitative support for those predictions in the observations. Figure 6a shows that the total $L_{\mathrm{mm}}$ in binary pairs marginally increases with their projected 


\section{Unbound Dynamical Encounters}

Flyby encounters between unbound stars and their disks are a natural extension of the dynamical interactions experienced in binaries (Clarke \& Pringle 1993). The probability for such encounters is enhanced at early times, where the cluster environment has a higher local stellar density (e.g., Bate 2018). Pfalzner (2013) predict that $\sim 1$ in 3 solar-type stars in an OB association experiences a close (100-1000 au) periastron passage within $1 \mathrm{Myr}$. These flybys can substantially perturb disk structures, including the creation of spiral arms or tidal bridges (Cuello et al. 2019b), truncation (Breslau et al. 2014), and warping or partial disruption (Xiang-Gruess 2016). The key demographic prediction from these encounters is that single stars in clusters with higher stellar densities should host smaller, less massive disks (de Juan Ovelar et al. 2012; Rosotti et al. 2014). While a direct test of that hypothesis is not yet tractable (due to the current focus of ALMA surveys on nearby loose associations), there are signs of potentially related morphological features in individual systems, including possible tidal extensions (Winter et al. 2018a) and spiral perturbations for disks in widely-separated binaries (Mayama et al. 2012; Rodriguez et al. 2018; Kurtovic et al. 2018).

separation (Jensen et al. 1994; Harris et al. 2012; Akeson \& Jensen 2014). That behavior is convolved with the $L_{\mathrm{mm}}-M_{*}$ relation: Akeson et al. (2019) found the same shape for that relation applies for the individual components of binaries, but with a normalization offset. The mean $L_{\mathrm{mm}}$ is $3-4 \times$ lower at the same $M_{*}$ for the binaries. Manara et al. (2019) provided more support for the truncation hypothesis by comparing mm continuum emission sizes for disks in analogous subsamples. Figure $6 \mathbf{b}$ demonstrates that $R_{\mathrm{mm}}$ for individual disks in binaries are $\sim 2 \times$ smaller than for a comparison set of disks around single stars.

However, Manara et al. (2019) found that the measured $R_{\mathrm{mm}}$ are too small compared with the truncation model predictions, given the projected separations (see also Harris et al. 2012). The discrepancy could point to eccentric orbits or indicate that the models are inappropriate. Those models presume co-planarity between the disks and stellar orbits, which is often not the case for the medium-separation binaries where $R_{\mathrm{mm}}$ estimates are tractable (Jensen \& Akeson 2014; Williams et al. 2014; Tobin et al. 2016; Brinch et al. 2016; Alves et al. 2019). Moreover, the models make predictions for the gas distribution, which is usually more extended than the solids (Section 2.2).

3.2.2. External Photoevaporation. Dynamical encounters are not the only environmental factors that alter disk structures. The intense radiation produced by massive stars can heat the outer regions of nearby disks until the sound speed exceeds the escape velocity, generating considerable mass loss in a wind (Hollenbach et al. 1994; Alexander et al. 2014). That externally-driven photoevaporative mass loss is validated with observations of ionization fronts associated with disks in the Orion Trapezium region (Johnstone et al. 1998; Störzer \& Hollenbach 1999). From a demographic perspective, photoevaporation models predict that disk sizes and masses should be lower in close proximity to massive stars. Indeed, the mean $L_{\mathrm{mm}}$ drops within $\sim 0.03 \mathrm{pc}$ of the massive star $\theta^{1}$ Ori C (Mann \& Williams 2009, 2010; Mann et al. 2014), corresponding to the region where ionization from its Lyman continuum radiation dominates (Johnstone et al. 1998). Eisner et al. (2018) found that $L_{\mathrm{mm}}$ and continuum sizes increase with distance from $\theta^{1}$ Ori $\mathrm{C}$, but are generally lower than for the disks in clusters without massive stars. That behavior is consistent with models that 

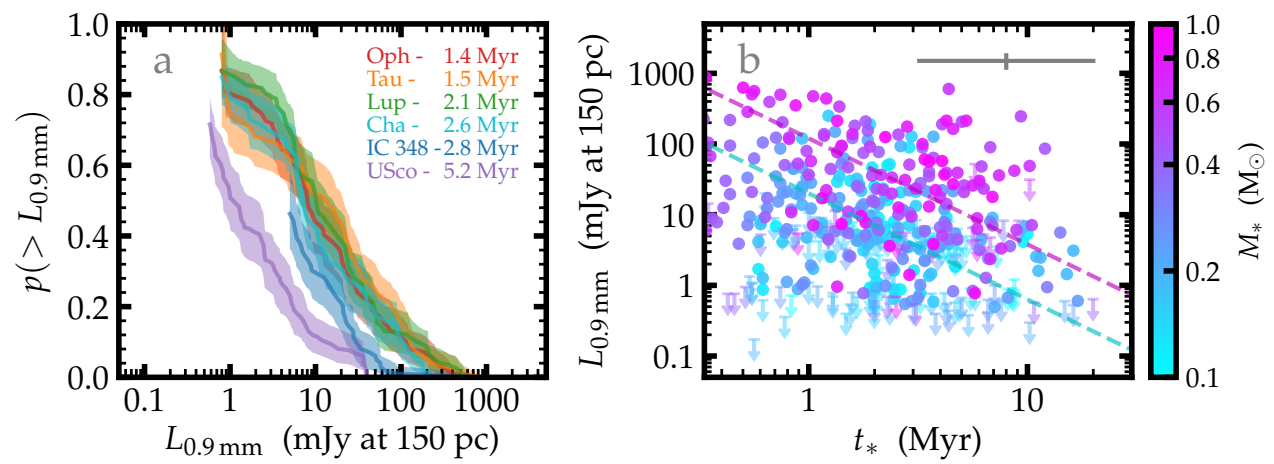

Figure 7

(a) Comparisons of $L_{\mathrm{mm}}$ distributions in star-forming regions with different mean ages. This is done in a Monte Carlo approach to control for $M_{*}$ : 500 distributions are drawn for each region, with each a collection of 100 measurements chosen such that the host masses follow the same mass function (known multiple systems are excluded). The colored bands show the $68 \%$ confidence intervals of those draws. There is a clear progression toward lower $L_{\mathrm{mm}}$ with mean cluster age (estimated from the parent samples). (b) A more direct, individualized examination of the $L_{\mathrm{mm}}-t_{*}$ relation, that better highlights the challenges of such comparisons due to the intrinsic scatter. Individual uncertainties are suppressed for clarity, but a mean error bar is shown in the top right. The color scale tracks the $M_{*}$-dependence; the dashed line shows a $L_{\mathrm{mm}} \propto t_{*}^{-1.5}$ scaling (note, those scalings are not fits to the data, just a rough estimate to guide the eye).

suggest a larger region of influence on disk structures from less energetic (far-ultraviolet) radiation fields (Facchini et al. 2016; Ansdell et al. 2017; van Terwisga et al. 2019).

\subsection{Evolutionary Signatures}

Much of the work on disk demographics focuses on the variations as a function of some metric of the elapsed time or evolutionary state of the system. One option is direct, considering how disk properties depend on their stellar host ages, $t_{*}$. While that seems natural, it is not trivial in practice because the ages are both imprecise and potentially inaccurate, due to biases in both the measurements and the models (e.g., Bell et al. 2013). With those caveats in mind, common practice is to compare the distributions of a given disk probe in young star clusters with a progression of mean ages. When controlling for $M_{*}$ and multiplicity, Figure $7 \mathbf{a}$ shows that the $L_{\mathrm{mm}}$ distribution shifts downward on $\sim 5$ Myr timescales (Barenfeld et al. 2017a; Ruíz-Rodríguez et al. 2018). A crude estimate of the decline suggests $L_{\mathrm{mm}} \propto t_{*}^{-1.5}$. There is evidence that the shape of the $L_{\mathrm{mm}}$ distribution changes, manifested as a steepening in the $L_{\mathrm{mm}}-M_{*}$ relationship over time (Pascucci et al. 2016; Barenfeld et al. 2017a). Figure $\mathbf{7 b}$ shows the same information in individual datapoints.

Some, perhaps all, of this evolution in $L_{\mathrm{mm}}$ is associated with changes in the continuum size-luminosity relationship: $R_{\mathrm{mm}}$ is generally smaller for disks in older clusters (Barenfeld et al. 2017b; Hendler et al. 2020). Such behavior indicates that the growth and migration of disk solids are key factors driving these demographic trends, rather than wholesale $M_{\mathrm{s}}$ changes (Tripathi et al. 2017; Rosotti et al. 2019a; Section 4). But analyses like these tell only part of the story. Focusing solely on systems that show excess infrared emission introduces a form of survivor bias by not accounting for the fact that the disk (infrared excess) fraction also decreases with $t_{*}$ (Haisch et al. 2001; Hernández et al. 2007). With 
that in mind, the combined effects of evolution are clearly under-estimated.

An alternative approach can help mitigate that survivor bias. The idea is to track how disks change as a function of their SED shape, an empirical diagnostic of the evolutionary state of the circumstellar material. Generally, this sequence where the SED peak moves progressively to shorter $\lambda$ reflects the dissipation of the envelope (Class $0 \rightarrow \mathrm{I} \rightarrow \mathrm{II}$ ) and disk (Class II $\rightarrow$ III; e.g., Williams \& Cieza 2011). The $L_{\mathrm{mm}}$ (or $M_{\mathrm{s}}$ ) distributions again shift downward along this evolutionary sequence (Andrews \& Williams 2007b; Sheehan \& Eisner 2017; Tychoniec et al. 2018; Williams et al. 2019). It is not easy to measure disk properties in the embedded phases (Class 0/I), due to both the technical challenge of disentangling emission from the envelope (Tobin et al. 2015) and the potential for younger disks to be intrinsically small (Segura-Cox et al. 2018; Maury et al. 2019). Most estimates find little evolution in $L_{\mathrm{mm}}$ during the Class 0 to I transition, even with a considerable decrease in envelope mass (Jørgensen et al. 2009; Segura-Cox et al. 2018; Andersen et al. 2019).

Despite some of the benefits of this latter evolutionary axis, it is difficult to contextualize the results without reference to a quantitative timeline. Moreover, there is potential to make unfair comparisons that are not able to control for orthogonal relationships (e.g., a $M_{*}$ dependence, since stellar properties for Class 0/I sources are unknown) or sample completeness (e.g., large mm Class III surveys are unavailable).

\section{THE EVOLUTION OF DISK SOLIDS}

The physical origins of the demographic behaviors that were outlined in the previous section are presumably closely related to the growth and migration of the disk solids. However, the vast scope and complexity of that evolution is daunting. To generate a population of planetesimals suitable for assembling a planetary system, the sub- $\mu \mathrm{m}$ dust grains incorporated into the disk at its formation epoch need to grow $>12$ orders of magnitude in size within a few Myr. This section highlights the basic theoretical framework developed to understand these processes (Section 4.1), explores the observational constraints (Sections 4.2 and 4.3), and considers the implications of some persistent obstacles (Section 4.4).

\subsection{Standard Theoretical Picture}

The two key elements required to model the evolution of disk solids are prescriptions for their coupling to the fluid motions of the gas (Nakagawa et al. 1986) and the outcomes of particle collisions (Güttler et al. 2010). Standard models start with small dust grains distributed homogeneously within a smooth gas disk, where the pressure $(P)$ decreases monotonically with $r$ and $z$. Turbulent diffusion is described with a simple viscosity prescription for fixed $\alpha_{\mathrm{t}}$. The small dust is well coupled to the gas, and so acquires low relative velocities through diffusive motions that result in gentle collisions that promote growth to porous aggregates (Henning \& Stognienko 1996; Dominik \& Tielens 1997). Those aggregates settle toward the midplane (Dubrulle et al. 1995), where the growth sequence continues. The material properties (internal structure, charge state, ice coating), sizes, and relative velocities of the impactor and target solids determine whether a collision is productive (mass transfer; Teiser \& Wurm 2009), neutral (bouncing; Zsom et al. 2010), or destructive (fragmentation, erosion; Birnstiel et al. 2010; Krijt et al. 2015). Simulations indicate that growth continues until collisions become destructive (Dullemond \& Dominik 2005) or the local particle population is depleted by radial migration (Takeuchi \& Lin 2002; Brauer et al. 2007; Birnstiel et al. 


\section{High-Dimensional Complexity in Particle Properties}

The limited scope of the metrics explored in Section 4.2 reflect the over-simplified emphasis in the literature. The reality is that many other factors can influence the absorption and scattering properties of the particles, and therefore the key observables (e.g., Min et al. 2016). This high-dimensional complexity includes mineralogical compositions (Henning \& Stognienko 1996; Cuzzi et al. 2014; Woitke et al. 2016), asphericity (Bertrang \& Wolf 2017), temperature-dependent refractive indices (Boudet et al. 2005), the methodology for mixing dielectric properties in composite particles (Birnstiel et al. 2018), and more sophisticated particle size distributions (Birnstiel et al. 2011), to name only a few. While these issues could change the details, the qualitative behaviors should be generally preserved. However, when confronted with subtle discrepancies or tensions (e.g., Section 4.3.4), a wider exploration of these other factors should be seen as a priority.

2009). For typical disk parameters, the latter effect dominates.

For a smooth disk, pressure support generates an additional outward force on a parcel of gas that effectively slows its orbital motion (Whipple 1972). The radial migration ("drift") of solids occurs once particles reach a size where they start to aerodynamically decouple from the gas; once disconnected from the pressure support of the gas, the particles spiral inwards toward the global maximum in $P$ (Weidenschilling 1977b). The timescales for that migration are much shorter than the collision timescales, thereby inhibiting further growth at that location (Takeuchi \& Lin 2005; Brauer et al. 2008). As a guide, drift is typically most efficient for pebbles ( $\mathrm{mm} / \mathrm{cm}$ sizes) at $r \approx 10-100 \mathrm{au}$.

The combined effects of growth and migration - both vertically (settling) and radially (drift) - profoundly influence the properties of disk solids (Testi et al. 2014). The simplest distillation of the key predictions in this standard framework is that disks should exhibit pronounced, negative spatial gradients (i.e., decreasing with $r$ and $z$ ) in their mean particle sizes and solids-to-gas mass ratios, such that larger solids at higher concentrations (relative to the gas) are found near the midplane (Dullemond \& Dominik 2004a; D'Alessio et al. 2006) and closer to the host star (Birnstiel et al. 2009, 2015; Birnstiel \& Andrews 2014).

\subsection{Metrics of Particle Properties}

In principle, those key predictions can be measured observationally. But designing the appropriate experiments and then interpreting the measurements requires a nuanced understanding of how particle properties are translated into disk tracers. The interactions of solid particles with radiation depend on their bulk properties, including compositions (Pollack et al. 1994), morphologies (Henning \& Stognienko 1996), and especially sizes (Miyake \& Nakagawa 1993) - but see also the box above. Those properties are encoded in the (absorption) opacities $\left(\kappa_{\nu}\right)$, albedos $\left(\omega_{\nu}\right)$, and polarizations $\left(\mathcal{P}_{\nu}\right)$ of the particle ensemble. ${ }^{1}$ Measurements of the thermal continuum and scattered light reflect the convolution of the physical conditions of the solids and the behaviors of $\left\{\kappa_{\nu}, \omega_{\nu}, \mathcal{P}_{\nu}\right\}$.

The morphology and size distributions for a population of solids have the most significant effects on the observables. Technically, morphologies are affected by both shape and internal

${ }^{1}$ The phase angle variations of $\omega_{\nu}$ and $\mathcal{P}_{\nu}$ also contain information about the particles. 

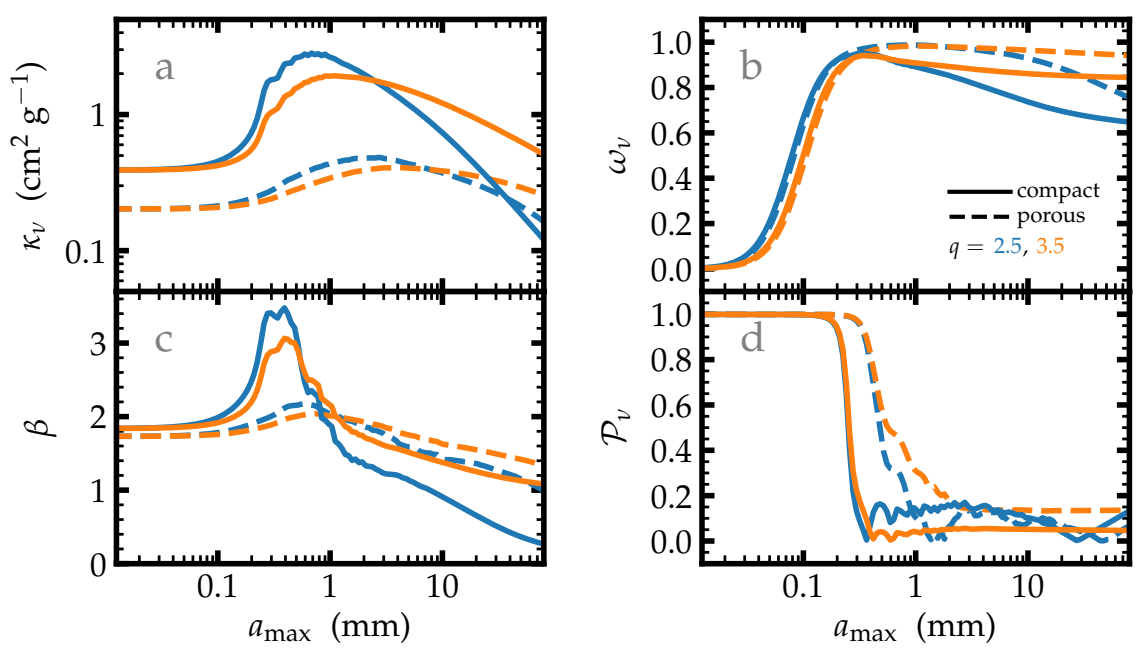

Figure 8

The variations of the $\lambda=1.3 \mathrm{~mm}$ (a) absorption opacity, (b) albedo (accounting for the mean scattering angle), (c) absorption opacity spectral index (between 1.3 and $3 \mathrm{~mm}$ ), and (d) polarization fraction as a function of the maximum particle size. Each panel contains four curves, showing different power-law size distributions ( $q=2.5$ in blue, $q=3.5$ in orange) and porosities (compact grains with $f_{\mathrm{s}}=1$ as solid, and $f_{\mathrm{s}}=0.5$ as dashed). These behaviors were calculated with a standard Mie scattering code for the assumptions outlined by Birnstiel et al. (2018).

structure (porosity), but the former is often ignored. The porosity is parameterized by a volume filling factor $f_{\mathrm{s}}$ (=1 for compact particles). Particle size distributions are usually approximated as power-laws, $n(a) \propto a^{-q}$ for sizes (particle radii) $a \in\left[a_{\min }, a_{\max }\right]$, with indices comparable to expectations for a collisional cascade $(q \approx 3.5$; Dohnanyi 1969) or a more top-heavy variant ( $q \approx 2.5$; e.g., Birnstiel et al. 2011).

Because of the (presumed) low optical depths, much of the work on particle properties in disks is conducted at $\mathrm{mm} / \mathrm{cm}$ wavelengths. There, $a_{\min }$ is irrelevant and the opacity spectrum can be approximated as a power-law, $\kappa_{\nu} \propto \nu^{\beta}$. Figure 8 illustrates how $\left\{\kappa_{\nu}, \beta\right.$, $\left.\omega_{\nu}, \mathcal{P}_{\nu}\right\}$ respond to the particle properties $\left\{a_{\max }, q, f_{\mathrm{s}}\right\}$ at $\lambda=1.3 \mathrm{~mm}$ for the assumptions of Birnstiel et al. (2018). The behavior at other wavelengths is qualitatively similar, with the main features shifted for an $a_{\max } \sim \lambda / 2 \pi$ scaling. When $a_{\max } \ll \lambda, \kappa_{\nu}$ is independent of size, $\beta$ is high ( $\sim 1.7$, as for the small dust grains in the ISM; Finkbeiner et al. 1999), and scattering is negligible $\left(\omega_{\nu} \approx 0\right.$, though $\mathcal{P}_{\nu}$ is high). When $a_{\max } \gg \lambda, \kappa_{\nu}$ decreases with $a_{\text {max }}$ at a rate that depends on $q$ (lower $q$ means a steeper fall-off; e.g., Ricci et al. 2010b), $\beta$ is lower (scaling roughly with $q$; Draine 2006), albedos are high (larger $q$ implies higher $\omega_{\nu}$ ), and $\mathcal{P}_{\nu}$ is low. When $a_{\max } \sim \lambda$, resonances drive up $\kappa_{\nu}, \beta$, and $\omega_{\nu}$, while $\mathcal{P}_{\nu}$ drops precipitously. Porosity dampens the resonant amplifications in $\kappa_{\nu}$ and $\beta$, but can enhance $\omega_{\nu}$ and $\mathcal{P}_{\nu}$, and generally modifies the $a_{\max } \gg \lambda$ behavior (Kataoka et al. 2014).

It is worthwhile to specifically address the apocryphal notion that (optically thin) $\mathrm{mm}$ continuum emission traces particles with $a \sim \lambda$. A more accurate statement is that the emission is most efficient in that case, since this corresponds to the resonant peak in $\kappa_{\nu}$ and therefore gives the most emission per mass. However, all sizes still contribute, and that creates an intrinsic ambiguity: $\kappa_{\nu}$ can be arbitrarily low if larger solids are present. An 
observational constraint on $\beta$ only sets a lower bound on $a_{\max }$, since $\beta$ effectively saturates once $a_{\max } \gg \lambda$. That, in turn, sets an upper bound on $\kappa_{\nu}$, and correspondingly a lower bound on the mass-related quantities (namely, $\Sigma_{\mathrm{s}}$ or $M_{\mathrm{s}}$ ).

\subsection{Measurements of Particle Growth and Migration}

4.3.1. Scattered Light and the Infrared SED. Optical and near-infrared images demonstrate that the starlight reflected from disk surfaces is typically faint (low $\omega_{\nu}$; Fukagawa et al. 2010), gray or red (Weinberger et al. 2002; Schneider et al. 2003), and forward-scattered (Quanz et al. 2011; Mulders \& Dominik 2012). Taken together, those properties indicate dust aggregates with $a_{\max } \gtrsim 10 \mu \mathrm{m}$ in disk atmospheres, representing the early steps in the growth sequence or possibly tracing collision fragments mixed up from the midplane. Similar conclusions are drawn from the shapes of solid-state emission features in the midinfrared, though isolating the inner disk with an interferometer is essential for robustly assessing the more processed grains located in the inner disk (van Boekel et al. 2004).

Direct measurements that trace the settling of dust aggregates toward the disk midplane are difficult due to the small intrinsic extent of the vertical dimension (with characteristic aspect ratios $z / r \lesssim 0.1$ ). Very high resolution mm continuum observations of edge-on disks are expected to provide decisive constraints on settling in the near future (e.g., Boehler et al. 2013; Louvet et al. 2018). For now the effects are identifiable with indirect probes, like the morphology of the infrared SED. Settling depletes particle densities relative to the gas in the disk atmosphere, reducing the infrared opacity and associated continuum emission below expectations from models that assume gas and dust are well-mixed (Dullemond \& Dominik 2004b; D'Alessio et al. 2006). The suppression of the infrared SED inferred from those models suggests that the dust-to-gas ratio is depleted $10-100 \times$ in disk atmospheres (e.g., Furlan et al. 2011). Analogous evidence can be retrieved from multiwavelength scattered light images: settling induces a vertical stratification of particle sizes $\left(a_{\max }(z)\right.$ is decreasing), and the corresponding gradient in $\omega_{\nu}$ makes the height of the scattering surface decrease with $\lambda$ (Pinte et al. 2007; Duchêne et al. 2010; McCabe et al. 2011; Muro-Arena et al. 2018).

4.3.2. Millimeter Continuum Spectrum. The mm continuum emission offers the most discriminating probes of particle properties near the disk midplane. In the optically thin limit, the intensity scales like $I_{\nu} \propto \kappa_{\nu} B_{\nu}(T) \Sigma_{\mathrm{s}}$. But since $\kappa_{\nu}$ cannot be determined uniquely, information about the particle properties is only accessible through the shape of the spectrum, quantified by the spectral index $\varepsilon$ (where $I_{\nu} \propto \nu^{\varepsilon}$ ), with $\varepsilon \approx \varepsilon_{\mathrm{Pl}}+\beta$ a sum of contributions from the Planck function $\left(B_{\nu} \propto \nu^{\varepsilon_{\mathrm{Pl}}}\right.$, where $\varepsilon_{\mathrm{Pl}} \approx 1.7-2.0$ for $\left.T>15 \mathrm{~K}\right)$ and the opacity spectrum $\left(\kappa_{\nu} \propto \nu^{\beta}\right)$. Resolved measurements of $\varepsilon(r, z)$ can test the predicted spatial segregation of particle sizes. Larger particles have smaller $\beta$ (Figure 8), and therefore smaller $\varepsilon$ : the hypothesis is that $\varepsilon$ increases (the spectrum steepens) with $r$ and $z$.

In practice, the disk-integrated spectral index $\alpha_{\mathrm{mm}}$ (where the flux density $F_{\nu} \propto \nu^{\alpha_{\mathrm{mm}}}$ ) is a much more common metric in the literature. As shown in Figure 9a, measurements find that $\alpha_{\mathrm{mm}} \approx 2-3$ in the $\lambda \approx 1-3 \mathrm{~mm}$ range (the mean $\alpha_{\mathrm{mm}}=2.2 \pm 0.3$ for $\lambda=0.9-1.3$ $\mathrm{mm}$ ), with a modest preference for steeper spectra at larger $L_{\mathrm{mm}}$ (Beckwith \& Sargent 1991; Andrews \& Williams 2005, 2007a; Ricci et al. 2010b,a; Ansdell et al. 2018). There are tentative hints that $\alpha_{\mathrm{mm}}$ also increases with $R_{\mathrm{mm}}$ and $M_{*}$ (as might be expected; see Sections 2.2 and 3.1), but the scatter is large and selection effects may dominate. Taken at face value, the measured $\alpha_{\mathrm{mm}}$ indicate shallow opacity spectra $(\beta \lesssim 1)$, and therefore large 

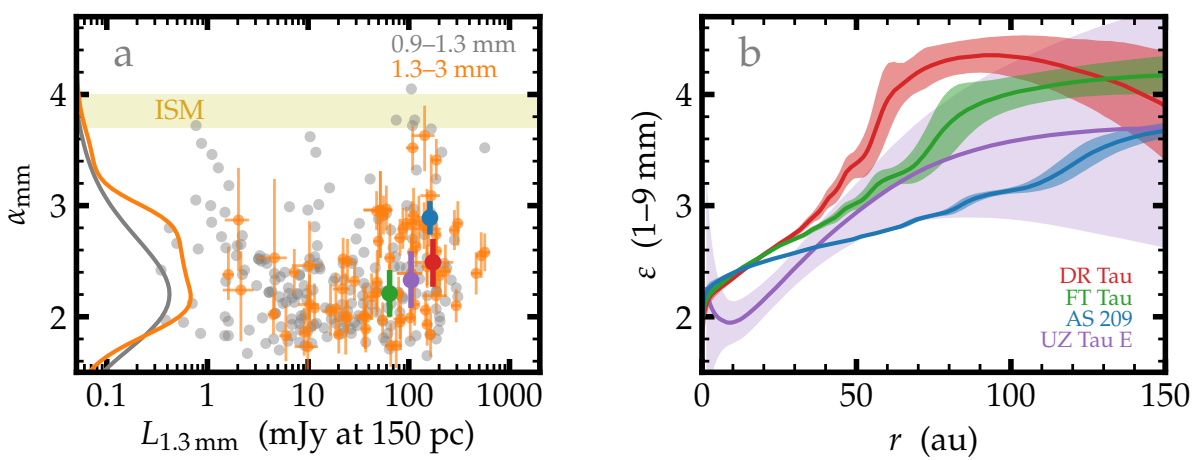

Figure 9

(a) The disk-integrated spectral indices, $\alpha_{\mathrm{mm}}$, as a function of $L_{\mathrm{mm}}$ at $1.3 \mathrm{~mm}$. Data were collected from the photometry surveys mentioned in the Figure 3 caption and supplemented with additional data when available (Ricci et al. 2010a,b, 2012, 2013, 2014; Lommen et al. 2010; Harris et al. 2012; Cieza et al. 2015; Testi et al. 2016; van der Plas et al. 2017; Cox et al. 2017; Pinilla et al. 2017c; Ward-Duong et al. 2018). Most spectra are much shallower than in the ISM, indicating particle growth and/or high optical depths. The $\alpha_{\mathrm{mm}}$ distributions (accounting for uncertainties) between $1.3-3 \mathrm{~mm}$ (orange) and $0.9-1.3 \mathrm{~mm}$ (gray, with $\sim 4 \times$ as many measurements, but less useful frequency leverage; uncertainties are suppressed for clarity, but are typically $\sim 0.4$ in $\alpha_{\mathrm{mm}}$ ) are shown along the ordinate axis. (b) The radial variations in the continuum spectral indices, between $\lambda=1$ and $9 \mathrm{~mm}$, inferred from modeling resolved multiwavelength continuum observations of four individual disks (Tazzari et al. 2016; Tripathi et al. 2018). Shaded areas show $68 \%$ confidence intervals around the posterior means marked with darker curves. These targets are marked with the corresponding colors in panel (a).

particles (e.g., $a_{\max } \gtrsim 10 \mathrm{~cm}$ for the models in Figure 8).

However, unresolved spectral index measurements gloss over some important complexities. Note that $\alpha_{\mathrm{mm}}$ is not a disk-averaged $\varepsilon\left(\alpha_{\mathrm{mm}} \neq\langle\varepsilon\rangle\right)$. The interpretation of $\alpha_{\mathrm{mm}}$ in the context of spatial variations in the continuum spectrum is ambiguous. Constraints on $\varepsilon(z)$ are difficult, again due to the intrinsically low aspect ratios of disks. That said, the rare limits on the vertical extent of the mm continuum are qualitatively consistent with the particle size segregation predicted by settling models with low $\alpha_{\mathrm{t}}$ (Guilloteau et al. 2016; Pinte et al. 2016). Measurements of $\varepsilon(r)$ that trace the combination of growth and radial drift are more practical. The common approach is to reconstruct $\varepsilon(r)$ from ratios of model fits to the multiwavelength $I_{\nu}(r)$ profiles (Isella et al. 2010; Guilloteau et al. 2011). Figure 9b shows some examples. A condensed alternative considers the wavelength dependence of the continuum sizes: an increasing $\varepsilon(r)$ implies a decreasing $R_{\mathrm{mm}}(\lambda)$ (Tripathi et al. 2018). In either case, such analyses infer that $\varepsilon(r)$ increases from $\sim 2$ in the inner disk (tens of au) to $\geq 3$ at larger $r$, corresponding to $\beta$ growing from $\sim 0$ to $\geq 1$ and $a_{\max }$ decreasing from $\gtrsim \mathrm{cm}$ to $\lesssim$ sub-mm sizes (Pérez et al. 2012, 2015a; Menu et al. 2014; Tazzari et al. 2016).

4.3.3. Polarization. Some complementary constraints on particle sizes are available from the linear polarization of self-scattered mm continuum emission (Hughes et al. 2009a; Kataoka et al. 2015). As illustrated in Figure 8, the albedo and polarization change precipitously in opposite directions near $a_{\max } \approx \lambda / 2 \pi$. The narrow shape of the product $\omega_{\nu} \mathcal{P}_{\nu}$ implies that polarization from scattering is only produced for a limited range of particle sizes. Measurements of the wavelength of peak $\mathcal{P}_{\nu}$ set a stringent limit on $a_{\text {max }}$. Resolved observations of 
polarized emission at $\lambda=0.9-1.3 \mathrm{~mm}$ find that the bulk morphologies of the polarization vectors are consistent with model predictions for scattering (Kataoka et al. 2016b; Yang et al. 2017b; Stephens et al. 2017; Hull et al. 2018; Bacciotti et al. 2018; Dent et al. 2019). For a few disks, multiwavelength $\mathcal{P}_{\nu}$ measurements indicate $a_{\max } \approx 0.1 \mathrm{~mm}$ (Kataoka et al. 2016a; Ohashi et al. 2018), considerably lower than inferred from the spectral indices.

4.3.4. Tension and the Optical Depth Caveat. The explanation for this apparent discrepancy in the characteristic $a_{\text {max }}$ inferred from the spectral indices and polarization properties of the mm continuum emission is not yet clear. One potential reconciliation is that the comparison itself could be misleading due to spatial variations in one or both of the tracers. For example, the outer regions of disks tend to have $\varepsilon \gtrsim 3$, which could be consistent with the polarization-based size constraints if much of the $\mathcal{P}_{\nu}$ behavior is produced at larger radii. But perhaps a simpler and more compelling possibility is that the assumption of low optical depths used to simplify the interpretation of the mm continuum emission is invalid. High optical depths suppress the continuum spectral index, with $\varepsilon \approx 1.5-2.5$, depending on the local temperature and the spectral variation of the albedo if scattering is important (Zhu et al. 2019; Liu 2019). There is still information about the particle sizes (in the $\tau_{\nu} \approx 1$ photosphere layer) available from $\varepsilon$ in this case, but the quantitative limits on $a_{\max }$ could indeed be very different than would be inferred in the optically thin limit. Though this possibility had previously been considered (Ricci et al. 2012), it is worth revisiting in the context of more detailed measurements of the disk emission (see Section 5).

4.3.5. Comparisons with Spectral Line Emission. Observational tests of the prediction that the solids-to-gas ratio decreases with $r$ are more challenging. Quantitative measurements of that ratio are impractical, given the ambiguities associated with measuring $\Sigma_{\mathrm{s}}$ and $\Sigma_{\mathrm{g}}$ (Section 2.3). Instead, investigations rely on a qualitative approach analogous to the SED constraints on the vertical variation of the dust-to-gas ratio discussed in Section 4.3.1. The strategy is to negate the hypothesis of a radially constant solids-to-gas ratio by demonstrating that such models cannot simultaneously explain the intensity profiles of both the mm continuum and a bright spectral line (Isella et al. 2007; Panić et al. 2009; Andrews et al. 2012). The argument is that the size discrepancy between the continuum and line emission (Figure $4 \mathbf{b}$ ) is an indirect indicator that $\Sigma_{\mathrm{s}}$ and $\Sigma_{\mathrm{g}}$ have different shapes.

Realistically, such a comparative analysis is not robust enough to be quantitative. There are legitimate concerns about radiative transfer effects, since the tracers being compared have very different optical depths (Hughes et al. 2008; Trapman et al. 2019). Moreover, it is not easy to disentangle the signatures of a solids-to-gas ratio that decreases with $r$ from the accompanying changes in $\kappa_{\nu}(r)$ (Facchini et al. 2017; Rosotti et al. 2019b). Trapman et al. (2019) argued that $R_{\mathrm{CO}} / R_{\mathrm{mm}} \gtrsim 4$ is an unambiguous indicator of growth and drift for smooth disks. The typically lower values of that ratio could still be consistent with that scenario (detailed modeling would be necessary), but might also reflect deviations from a smooth gas disk, where $R_{\mathrm{mm}}$ is effectively increased by slowed particle migration at local $P$ maxima while $R_{\mathrm{CO}}$ is unaffected (see Section 5).

\subsection{Toward Planetesimals}

The measurements outlined above are in good qualitative agreement with the standard theoretical predictions for the evolution of disk solids. This empirical support suggests that 
the basic physical ingredients in the models are appropriate. However, there are two important quantitative problems with the framework. The first is subtle: the predicted spatial segregation of particle sizes is generically more extreme than is implied by measurements of $\varepsilon(r)$ (e.g., Tripathi et al. 2018). The same problem appears as an over-prediction of the $\alpha_{\mathrm{mm}}$ distribution with respect to observations (Birnstiel et al. 2010; Pinilla et al. 2013), and a difficulty in reproducing the high end of the $R_{\mathrm{mm}}-L_{\mathrm{mm}}$ correlation (Tripathi et al. 2017; Rosotti et al. 2019a). Put simply, the predicted evolution is too fast to account for the data. The second problem is related, but more striking: the models do not produce planetesimals, or even $\gtrsim$ meter-sized bodies, within the timeframe associated with disk dispersal $(\sim 5-10$ Myr). Inside a few au, this latter issue is perhaps associated with incomplete physics in the models (Laibe et al. 2012; Okuzumi et al. 2012; Windmark et al. 2012a,b).

Ultimately, both of these problems are consequences of the predicted (high) efficiency for the radial migration of solids. The next section reconsiders an elegant solution to both problems, achieved by relaxing the standard assumption of a smooth gas disk.

\section{SUBSTRUCTURES}

Until recently, nearly all of the constraints on disk structures were derived from observations with relatively coarse spatial resolution, $\gtrsim 15-20$ au. As is frequently the case, improved facilities and instrumentation have precipitated a dramatic shift in the field, with a new emphasis on the prevalence of fine-scale features, or substructures, in these disks. Despite the narrowed focus on these details, important new insights have emerged that are re-shaping how disk properties are interpreted and contextualized more generally.

\subsection{Resolving the Drift Dilemma}

Substructures can reconcile the two fundamental problems associated with the migration of solids in the classical theory (Section 4.4). To explain how, it helps to revisit the cause of the migration. The force balance between gravity, rotation, and pressure support determines the orbital motion of the gas disk. The contribution associated with pressure support is proportional to the gradient $\mathrm{d} P / \mathrm{d} r$. The standard assumption of a smooth, monotonically decreasing $P(r)$ implies that $\mathrm{d} P / \mathrm{d} r$ is always negative, and therefore the gas orbits at subKeplerian velocities. For solids that decouple from the gas, drag extracts orbital energy and imparts a radial velocity directed inwards, toward the $P$ maximum at the inner disk edge. The key problem is that this radial drift is too efficient (Takeuchi \& Lin 2002, 2005).

However, if $P(r)$ is not monotonic but instead has local maxima, there are corresponding modulations to the gas dynamics with striking consequences. Exterior to a local maximum, the standard physical scenario applies: $\mathrm{d} P / \mathrm{d} r<0$, gas velocities are sub-Keplerian, and drifting particles move inwards. But just interior to a maximum, $\mathrm{d} P / \mathrm{d} r>0$ and the gas motion is super-Keplerian. In that case, particles are instead pushed outwards. Interactions with this perturbed gas flow drive particle migration to converge toward the local pressure maximum. At the maximum there is no pressure gradient ( $\mathrm{d} P / \mathrm{d} r=0$ by definition), so the gas motion is Keplerian and the solids do not drift. A sufficiently steep $P$ modulation with limited diffusion can effectively "trap" solids by slowing or halting their migration.

These substructures in the gas pressure distribution are essential ingredients for reconciling the drift and planetesimal formation timescale problems. If distributed throughout the disk, perturbations to $P(r)$ can alleviate the drift timescale problem by stalling particle 
migration (Pinilla et al. 2012a, 2013). Moreover, the resulting localized particle concentrations can attain solids-to-gas ratios that approach unity (e.g., Yang et al. 2017a), thereby creating favorable conditions for the rapid conversion of pebbles into planetesimals through the streaming instability (Youdin \& Goodman 2005; Johansen et al. 2007) or direct gravitational collapse (Goldreich \& Ward 1973; Youdin \& Shu 2002).

\subsection{Potential Physical Origins}

The hypothesis that substructures are both elemental disk characteristics and fundamental aspects of the planet formation process is agnostic about their physical origins. However, a remarkable variety of ways to generate substructures that trap migrating solids (or otherwise perturb their migration) have been proposed in the literature. The discussion below highlights these mechanisms, coarsely grouped into three general categories. The schematics in Figure 10 illustrate how some of these mechanisms are manifested as small-scale perturbations to the distributions of gas and solids in the disk.

5.2.1. Fluid Mechanics. The physical conditions in disks are subject to various (magneto)hydrodynamic flows and instabilities that locally perturb gas pressures. For example, the mechanics of disk dispersal itself can substantially reshape the disk structure. Depending on the mass-loss profile, simulations of outflows from MHD-driven winds (Suzuki et al. 2016; Takahashi \& Muto 2018) or photoevaporative flows (Alexander et al. 2014; Ercolano \& Pascucci 2017) predict a ring-shaped pressure maximum at $\sim$ tens of au, with a depleted (or even empty) cavity interior to it (see Figure 10a).

Even without imposing a special evolutionary state, generic fluid mechanics properties likely also play roles in substructure formation. Turbulence generates stochastic $P$ modulations that concentrate particles and diminish their drift rates (Cuzzi et al. 2001; Pan et al. 2011). That behavior might predict a disk mottled with substructures on the eddy scale $\left(\lesssim H_{p}\right)$, but many simulations demonstrate that MHD turbulence tends to self-organize into more coherent features in the $P$ distribution, including spirals (Heinemann \& Papaloizou 2009; Flock et al. 2011) and axisymmetric undulations (Johansen et al. 2009; Dittrich et al. 2013). The latter are produced when the gas dynamics are modified by spontaneous, concentric concentrations of magnetic flux, which repel gas from regions of peak magnetic stress and pile it up at neighboring annuli (Uribe et al. 2011; Bai \& Stone 2014; Simon \& Armitage 2014; Béthune et al. 2016; Suriano et al. 2017, 2018). These zonal flows create narrow $\left(\Delta r \approx\right.$ few to $10 H_{p}$ ) depletions (gaps) and enhancements (rings) in $P(r)$ at $r \approx$ tens of au that are expected to trap and concentrate solids (see Figure 10b).

In very dense regions of the disk, the ionization rate can be diminished enough to stifle turbulence from the MRI (Gammie 1996). The radial variation of $\alpha_{\mathrm{t}}$ into such a "dead zone" modifies the gas flow and can thereby produce a strong, axisymmetric maximum in $P(r)$ at the laminar/turbulent boundary (Regály et al. 2012; Dzyurkevich et al. 2013). These transitions at dead zone boundaries can generate vortices through the Rossby wave instability (Lovelace et al. 1999; Lyra et al. 2009), resulting in radially narrow $\left(\Delta r \approx H_{p}\right)$ but azimuthally extended $(\Delta \theta \gtrsim \pi / 2)$ pressure maxima (Lyra \& Lin 2013; Baruteau \& Zhu 2016). There are alternative ways to cultivate vortices, including baroclinic instabilities (Klahr \& Bodenheimer 2003), which can be amplified by feedback from the solids (LorénAguilar \& Bate 2015, 2016), and the vertical shear instability (Richard et al. 2016). Vortices attract and concentrate migrating solids, making them especially compelling sites for plan- 

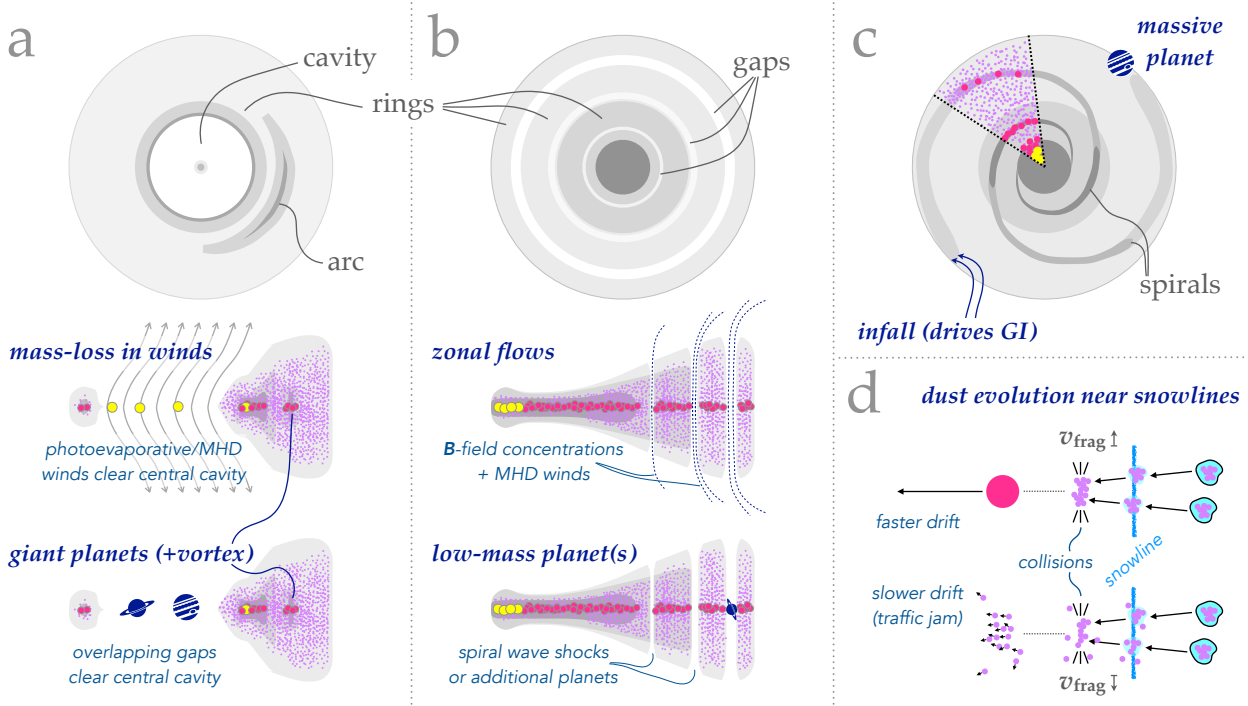

Figure 10

Schematic illustrations of the substructures generated by various physical mechanisms. As in Figure 1, grayscale denotes gas densities $(\propto P)$ and representative solid densities are marked with exaggerated symbol sizes and colors. (a) A schematic of a ring/cavity substructure morphology with a pronounced arc feature generated by a vortex. The two side views represent the behavior for a disk with substantial mass-loss in a photoevaporative or MHD-driven wind (top) or a series of giant planets (bottom), both of which effectively diminish $\Sigma_{\mathrm{g}}$ in a central cavity. The sharp density contrast at the cavity edge can trap particles in a ring and potentially generate a vortex. (b) A schematic of the ring/gap substructure morphology, with similar behavior produced by the magnetic field concentrations inherent in MHD zonal flows (top) and the perturbations from interactions between lower mass planets and a relatively inviscid disk (bottom). (c) A simplified diagram of the spiral wave perturbations that could be produced by the global GI driven by remnant envelope infall or tidal interactions with a massive (external) planetary companion. (d) A cartoon highlighting two representative outcomes for the evolution of icy aggregates as they migrate across a volatile condensation front. The top behavior shows the case where ice loss to sublimation enhances $v_{\text {frag }}$, and thereby promotes growth and drift; the bottom behavior is the opposite, resulting in a pileup of small, bare grains.

etesimal formation (Barge \& Sommeria 1995; Klahr \& Henning 1997; Klahr \& Bodenheimer 2006), and can also imprint long-lasting rings and gaps in $\Sigma_{\text {s }}$ (e.g., Surville et al. 2016).

For sufficiently dense and cold configurations, self-gravity can drive a global gravitational instability (GI) that imposes a large-scale spiral pattern onto the disk structure (see Figure 10c; Toomre 1964; Boss 1997). A more unstable disk produces lower order modes (fewer arms) and more open (loosely-wrapped) patterns (e.g., Kratter \& Lodato 2016). The pressure peaks of the spiral waves concentrate and foster the growth of drifting particles (Rice et al. 2004; Dipierro et al. 2015). At early evolutionary stages, asymmetric envelope accretion could drive the global GI (Laughlin \& Bodenheimer 1994; Tomida et al. 2017; Hall et al. 2019). That infall process could also create vortices (Bae et al. 2015), generate an unstable shock that propagates in spiral density waves (Lesur et al. 2015), or magnetically imprint over-densities in concentric rings (Suriano et al. 2017).

Various other modes of gas-particle coupling could also precipitate substructures in $\Sigma_{\mathrm{s}}$ 
and perhaps accelerate planetesimal formation if the solids-to-gas ratio is enhanced. Two interesting examples are cases where ring-shaped particle over-densities are self-induced by a dynamical feedback (solids on gas) process (Drążkowska et al. 2016; Gonzalez et al. 2017) or a viscous feedback instability where solid enhancements diminish $\alpha_{\mathrm{t}}$ and perturb the gas dynamics (Wünsch et al. 2005; Dullemond \& Penzlin 2018). A special focus has been on the secular GI, which occurs when gas drag slows the self-gravitational collapse of solids enough to shear out the over-densities into narrow rings (Shariff \& Cuzzi 2011; Youdin 2011). Simulations of the secular GI find $\sim H_{p}$-scale (perhaps clumpy) concentric peaks in $\Sigma_{\mathrm{s}}$, provided the turbulence is low $\left(\alpha_{\mathrm{t}} \lesssim 10^{-3}\right.$; Takahashi \& Inutsuka 2014, 2016).

Obviously a remarkable variety of mechanisms in the broader fluid dynamics category can theoretically generate perturbations in $P\left(\right.$ or $\left.\Sigma_{\mathrm{s}}\right)$ that are sufficient to mitigate the drift problem and promote the local concentration of solids. In this general picture, the disk substructures produced by these internal, naturally-occurring mechanisms represent the fundamental initial conditions for planetesimal (and thereby planet) formation.

5.2.2. Dynamical Interactions with Companions. Gravitational (tidal) perturbations by a companion are a less subtle means of modifying disk properties, but are capable of generating a similar diversity of substructures as in the fluid mechanics category. The emphasis here is on planets, although analogous effects are relevant for stellar binaries (Section 3.2). Once it has accumulated sufficient mass, a planetary companion interacts with the disk, generating spiral shocks that transfer angular momentum and repel disk material away from its orbit (Lin \& Papaloizou 1979, 1986; Goldreich \& Tremaine 1980). That perturbation can clear an annular gap in $\Sigma_{\mathrm{g}}$, with a width and depth that depend on the planet mass and the local turbulent diffusion and thermal structure of the gas disk (Kley \& Nelson 2012). The pressure maxima produced outside the gap, around the planetary orbit, can trap drifting solids (Rice et al. 2006; Paardekooper \& Mellema 2006; Zhu et al. 2012).

More dramatic perturbations to disk structures are produced by more massive (giant) planetary companions $\left(\gtrsim M_{J u p}\right)$. Systems of multiple giant planets can have overlapping gaps that deplete $\Sigma_{\mathrm{g}}$ over a wide radial range (see Figure 10a; Dodson-Robinson \& Salyk 2011; Zhu et al. 2011), and may excite vortices or eccentric modes that generate strong azimuthal asymmetries in the pressure structure (Kley \& Dirksen 2006; Ataiee et al. 2013; Zhu et al. 2014). If the companion orbit is inclined with respect to the disk plane, it can warp (Nealon et al. 2018) or even dynamically isolate ("break") the disk into components with very different orientations (Owen \& Lai 2017; Zhu 2019). Much lower mass planets $\left(\gtrsim \mathrm{M}_{\oplus}\right)$ can still generate substructures in $\Sigma_{\mathrm{s}}$, even if they only weakly perturb $P$ (see Figure 10b; Dipierro et al. 2016; Rosotti et al. 2016). If turbulence is suppressed enough that the disk is essentially inviscid, low-mass planets can make a distinctive W-shaped radial variation in the particle distribution (with the planet orbit at the central peak; Dong et al. 2017) and drive secondary and tertiary spiral arms that deposit angular momentum far interior to the planet orbit in near-circular shocks that also perturb $\Sigma_{\mathrm{s}}$ (Bae et al. 2017; Bae \& Zhu 2018). Giant planets at large disk radii can also foster large-scale $m=2$ spiral modes interior to their orbits (Figure 10c; Zhu et al. 2015; Dong et al. 2015).

5.2.3. Condensation Fronts. Substructures in $\Sigma_{\mathrm{s}}$ can also be induced without local pressure maxima (see Section 5.2.1). A popular example is associated with the sublimation of icy particles as they migrate across condensation fronts (snowlines). In that scenario, three factors are relevant to consider. First, ice sublimation is a net mass loss for the solids, and 
therefore $\Sigma_{\mathrm{s}}$ is depleted inside a snowline (Stammler et al. 2017) over a radial range that depends on the coagulation timescales; efficient growth implies a narrow range. Second, gas that has been liberated from ices can be mixed back across the snowline and re-condensed (Stevenson \& Lunine 1988; Cuzzi \& Zahnle 2004; Ros \& Johansen 2013; Ros et al. 2019). This might help enhance particle growth and therefore $\Sigma_{\mathrm{s}}$ in a zone outside the snowline, with a width that depends on diffusion and migration rates.

The third factor is perhaps most significant. Ices can change the effective particle strengths (parameterized by the critical velocity for fragmentation, $v_{\text {frag }}$ ), and thereby affect collision outcomes. Pinilla et al. (2017b) considered how molecular bonds in ices affect

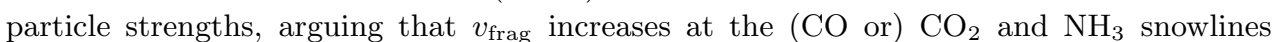
and decreases at the $\mathrm{H}_{2} \mathrm{O}$ snowline. This results in enhancements (depletions) of larger (smaller) particles between the $\mathrm{H}_{2} \mathrm{O}$ and ( $\mathrm{CO}$ or) $\mathrm{CO}_{2}$ snowlines, although diffusion $\left(\alpha_{\mathrm{t}}\right)$ affects the details (analogous to the top part of Figure 10d). If $v_{\text {frag }}$ decreases across a snowline, collisions can become destructive and the smaller fragments drift more slowly; the associated congestion increases $\Sigma_{\mathrm{s}}$ like a traffic jam (Birnstiel et al. 2010; Saito \& Sirono 2011). Okuzumi et al. (2016) argued that sintering during coagulation (Sirono 2011) diminishes $v_{\text {frag }}$, and therefore can enhance $\Sigma_{\mathrm{s}}$ due to the reduced migration rates of small fragments, in narrow zones outside the snowlines of even rare volatile species. These latter scenarios are illustrated in the bottom part of Figure 10d, although the sintering case would be shifted beyond the snowline (i.e., to the right in that diagram).

The interplay between these factors is complex (e.g., Ciesla \& Cuzzi 2006; Estrada et al. 2016), and likely complicated further by feedback reactions where particle accumulations affect the gas or solid dynamics (Dra̧żkowska \& Alibert 2017; Schoonenberg \& Ormel 2017; Gárate et al. 2019). The potential outcomes are diverse. However, the fundamental link to the disk temperatures means that the substructures associated with these mechanisms occur at special locations, and should be concentric and symmetric (presuming $T$ is dominated by a radial gradient). In some sense, these limits to the flexibility of predictions from this idea could be helpful for observational tests, at least compared to the broad universe of options available from the fluid dynamics effects or planet-disk interactions outlined above.

\subsection{Signatures of Substructures}

Given the myriad physical processes capable of perturbing $P$ and/or $\Sigma_{\mathrm{s}}$, it is reasonable to expect that any given disk is riddled with substructures. To find them and assess their origins, demographic dependences, and general roles in disk evolution and planet formation, measurements that characterize the forms, locations, sizes, and amplitudes of those features are crucial. Some generic predictions about the properties of substructures can serve as useful guides for designing observations. A stable perturbation to $P$ can have a characteristic size as small as $H_{p}$ (e.g., Dullemond et al. 2018). For a standard disk temperature profile, $H_{p} / r \approx 0.05-0.10$. That implies substructures might subtend only $\sim 5-50$ milliarcseconds for projected separations of $\sim 0.05-0.5$ arcseconds from the host star of a typical disk target $(d \approx 150 \mathrm{pc})$. Short-lived, stochastic features in the gas and the spatial concentrations of solids embedded in local pressure maxima could be even smaller. Pressure perturbations with $\gtrsim 20 \%$ amplitudes could be sufficient to trap drifting solids (Pinilla et al. 2012b). That sets a crude benchmark on the sensitivity goals, although a focus on the strongly amplified signal from trapped solids can substantially improve the search yields.

Obviously, those predictions foreshadow a challenging observational task. However, the 
high-fidelity datasets at novel resolutions that have become available over the past five years have enabled a first detailed look at disk substructures. The next sections explore some general properties and physical insights about such features from these initial measurements.

5.3.1. Morphology. High resolution images of disks in their optical/infrared scattered light or mm continuum emission have identified substructures and characterized their morphologies at effectively all spatial scales down to the current resolution limits, $\sim 1-5$ au (Zhang et al. 2016; Garufi et al. 2018; Andrews et al. 2018b; Long et al. 2018b). Figure 11 shows a gallery of images that highlights their morphological diversity. While there are some subtle complexities, and a considerable overlap that indicates more of a continuum of substructure patterns, it is reasonable to group the morphological types into four broad categories.

Ring/Cavity. This is the canonical morphology for a "transition" disk (Espaillat et al. 2014 ), with a primary narrow ring (usually peaking at $r \approx$ tens of au, though that is likely a selection bias) that encircles a depleted cavity (Piétu et al. 2007; Brown et al. 2009; Andrews et al. 2011). The cavities are usually, but not always, cleared enough to depress the infrared SED (Calvet et al. 2002; Espaillat et al. 2007; van der Marel et al. 2016c). Disks with this morphology offer the best observational evidence for particle traps in local $P$ maxima, given their amplified (narrow, bright) concentrations of $\mathrm{mm} / \mathrm{cm}$ continuum emission (Pinilla et al. 2012b, 2018b) and the lower amplitude and more spatially extended perturbations (both inside the cavity and to larger $r$ ) of line emission (tracing gas; Hughes et al. 2009b; van der Marel et al. 2015b, 2016b) and scattered light (probing small grains that are well coupled to the gas; Dong et al. 2012; Mayama et al. 2012; Villenave et al. 2019).

Rings/Gaps. This refers to a concentric, axisymmetric pattern of alternating intensity enhancements (rings) and depletions (gaps). It is the most common substructure morphology identified in the mm continuum (ALMA Partnership et al. 2015; Andrews et al. 2016; Huang et al. 2018b; Long et al. 2018b) and scattered light (Quanz et al. 2013; Akiyama et al. 2015; Rapson et al. 2015; Ginski et al. 2016; de Boer et al. 2016; Avenhaus et al. 2018). There are clear variants within this category, with options spanning from cases where the entire disk can be decomposed into narrow gaps and rings (e.g., CI Tau, Clarke et al. 2018; AS 209, Guzmán et al. 2018) to a single gap that separates an inner emission core from an outer ring (e.g., V883 Ori, Cieza et al. 2016; DS Tau, Long et al. 2018b).

Arcs. Non-axisymmetric substructures seem to be rare, although some disks exhibit arc features that span a limited range of azimuth. These arcs can be manifested as a partial ring around a central cavity, where the brightness asymmetry can range from severe $(\gtrsim 100 \times$; van der Marel et al. 2013; Casassus et al. 2013) to mild ( $\sim 2 \times$; Isella et al. 2013; Pérez et al. 2014; Loomis et al. 2017). Or, they can appear as additional substructures, located exterior to a ring/cavity morphology (Marino et al. 2015; van der Marel et al. 2016a; Kraus et al. 2017; Boehler et al. 2018) or in a gap (Isella et al. 2018; Pérez et al. 2018b).

Spirals. Large-scale spiral patterns are most prevalent in scattered light images, ranging from pronounced $m=2$ modes with modest brightness asymmetries and a relatively open morphology (Muto et al. 2012; Grady et al. 2013; Akiyama et al. 2016) to more intricate, tightly-wrapped, and asymmetric structures (Hashimoto et al. 2011; Avenhaus et al. 2014; Garufi et al. 2016; Monnier et al. 2019). There are only three known examples of disks around single star hosts that exhibit a spiral pattern in the mm continuum (Pérez et al. 2016; Huang et al. 2018c), in each case with a large, symmetric, two-armed pattern. Extended, complex spirals have been identified in spectral line emission in three (different) cases (Tang 


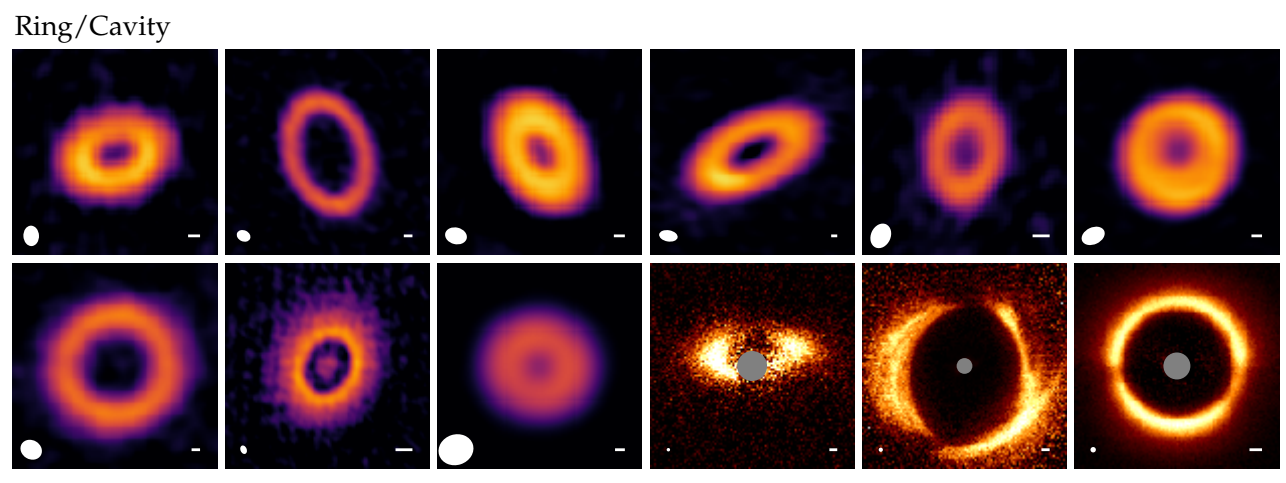

Rings/Gaps
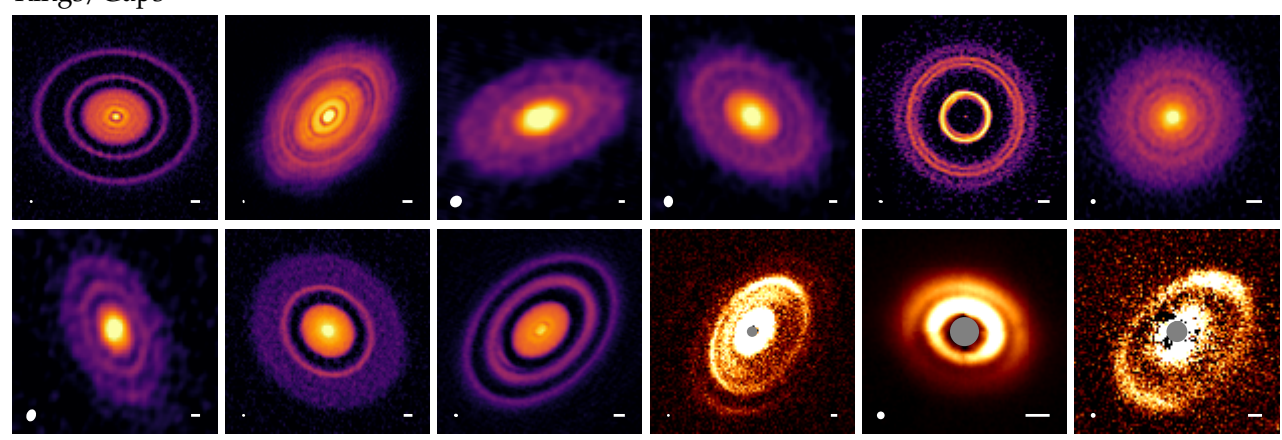

Arcs
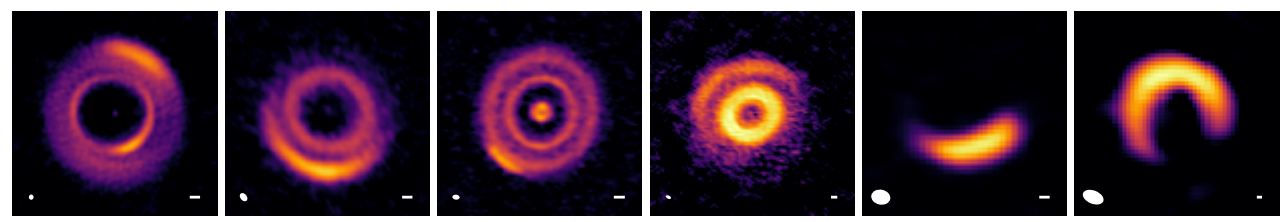

Spirals
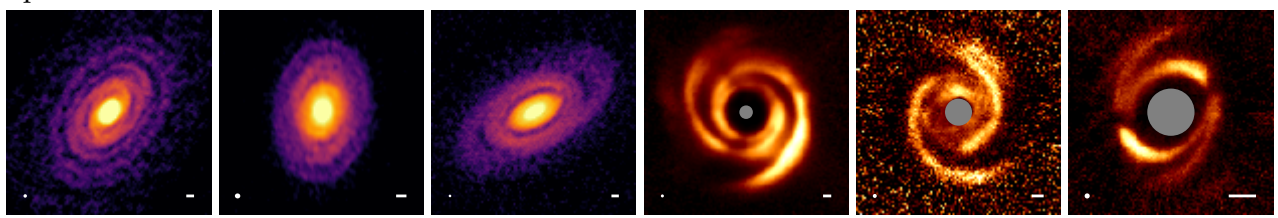

Figure 11

see following page.

et al. 2012, 2017; Christiaens et al. 2014; Teague et al. 2019).

Of course, individual disks can include features with multiple morphological types. Some examples were mentioned above for arc shapes, but there are also cases where rings, gaps, and a cavity (e.g., DM Tau, Kudo et al. 2018; Sz 129, Huang et al. 2018b) or rings, gaps, and spirals (Huang et al. 2018c) co-exist in the same disk and for the same observational tracer. Moreover, that mixing of morphological types can be striking when comparing 
Figure 11

A gallery of disk substructure morphologies; the color maps for mm continuum and infrared scattered light match those in Figure 2. Resolutions are marked with white ellipses in the lower left corners of each panel; 10 au scalebars are shown in the lower right corners. The mm continuum images are shown with an asinh stretch to $\sim 90 \%$ of their peaks. The scattered light images use a linear stretch, sometimes including an $r^{2}$ scaling, with the intent of matching the dynamic range shown in the literature: gray circles mark their coronagraphic spots (or bad pixel regions). Ring/Cavity images, from left to right: (top) CIDA 9 (Long et al. 2018b), Sz 91 (Tsukagoshi et al. 2019a), SR 24 S (Pinilla et al. 2017a), HD 34282 (van der Plas et al. 2017), IP Tau (Long et al. 2018b), SR 21 (van der Marel et al. 2018b); (bottom) RX J1604.3-2130 (Pinilla et al. 2018b), DM Tau (Kudo et al. 2018), DoAr 44 (Pinilla et al. 2018b), IRS 48 (Follette et al. 2015), HD 142527 (Avenhaus et al. 2014), and RX J1604.3-2130 (Pinilla et al. 2018c). Rings/Gaps images: (top) AS 209 (Guzmán et al. 2018), HL Tau (ALMA Partnership et al. 2015), V1094 Sco (van Terwisga et al. 2018), DL Tau (Long et al. 2018b), HD 169142 (Pérez et al. 2019a), RU Lup (Andrews et al. 2018b); (bottom) GO Tau (Long et al. 2018b), Elias 24 (Andrews et al. 2018b), RX J1852.3-3700 (Villenave et al. 2019), RX J1615.3-3255 (Avenhaus et al. 2018), V4046 Sgr (Avenhaus et al. 2018), and HD 163296 (Monnier et al. 2017). Arcs images: MWC 758 (Dong et al. 2018c), SAO 206462 (Cazzoletti et al. 2018), HD 143006 (Pérez et al. 2018b), HD 163296 (Isella et al. 2018), V1247 Ori (Kraus et al. 2017), HD 142527 (Casassus et al. 2013). Spirals images: IM Lup, WaOph 6, Elias 27 (all from Huang et al. 2018c), SAO 206462 (Stolker et al. 2017), MWC 758 (Benisty et al. 2015), and HD 100453 (Benisty et al. 2017).

images of the same disk in different tracers: the MWC 758 and SAO 206462 disks are favorite examples, showing spirals in scattered light (Benisty et al. 2015; Stolker et al. 2016), but rings, gaps, cavities, and arcs in the mm continuum (Dong et al. 2018c; Cazzoletti et al. 2018). This could be the hallmark of multiple mechanisms operating simultaneously, with different processes manifesting more clearly in tracers of the gas or the solids, or it could be indicative of a changing morphology as a function of altitude. There are some tentative preferences for certain morphological types as a function of $M_{*}$, age, and perhaps other demographic properties (Garufi et al. 2018), but selection effects are still a considerable problem; an unbiased census for substructures is not yet available.

5.3.2. Locations, Sizes, and Amplitudes. The ring/gap substructures in disks are found at essentially any radial location, from the resolution limit (a few au) out to the detection threshold ( $\lesssim 300 \mathrm{au}$; Huang et al. 2018b; van Terwisga et al. 2018). Ring/cavity and arc substructures are preferentially identified at larger $r$ ( tens of au), although that is likely a resolution bias (Huang et al. 2018b; Pinilla et al. 2018a). There are no obvious relationships between substructure locations and host properties (Pinilla et al. 2018a; Long et al. 2018b; Huang et al. 2018b; van der Marel et al. 2019). There is a propensity to find more distant substructures in larger disks, but it is not clear if this is a physical connection (those disks are larger and brighter because they produced more distant substructures) or a trivial artifact. First, more distant substructures should be larger (higher $H_{p}$ ) and therefore easier to find. And second, even if substructures exist at the same large $r$ for disks with smaller $R_{\mathrm{mm}}$, there is no continuum emission at $r \gg R_{\mathrm{mm}}$ to trace them; they would likely be missed. With those considerations in mind, the current suite of observations are consistent with the idea that there are no special locations essential for substructure formation.

The observed radial separations between neighboring ring or gap substructures in the $\mathrm{mm}$ continuum (peak-to-peak or trough-to-trough) span the range $\Delta r / \bar{r} \approx 0.2-0.5$ (where $\bar{r}$ is the midpoint between two features; Huang et al. 2018b), corresponding to characteristic spacings $\Delta r \approx 2-10 H_{p}$. The low end of that range is uncertain, since more compact 
spacings (especially in the inner disk) can be missed with limited resolution. A few disks include substructure pairings that have spacings commensurate with mean-motion resonances (Huang et al. 2018b). The two-dimensional nature of spiral substructures makes it difficult to define spacings (or locations). The pitch angles of two-armed spirals are typically $\sim 10$ $20^{\circ}$ (perhaps with modest radial gradients) in both scattered light (Muto et al. 2012; Yu et al. 2019) or mm continuum (Huang et al. 2018c) measurements. Simulations predict that the scattered light spirals should be more open than the mm continuum for a given disk, due primarily to the vertical temperature gradient (the scattered light tracers a higher, and therefore warmer, layer; Juhász \& Rosotti 2018). It is possible that more tightly-wound configurations could be mistaken for rings or arcs.

Measurements of substructure widths are difficult because most features are not well resolved. The broader rings/gaps in the mm continuum have $\mathrm{FWHM} / r \approx 0.1-0.5$, implying widths $\approx H_{p}$ (Huang et al. 2018b; Dullemond et al. 2018; Long et al. 2018b). The radial widths of most gaps, arcs, and spirals (i.e., in cuts perpendicular to tangent points) are $\lesssim 5-$ $10 \mathrm{au}$. The characteristic size distribution of substructures clearly extends below current resolution limits, but inferences of sub-resolution feature widths should be regarded with a healthy skepticism since they are strongly dependent on the adopted model prescription. There is more diversity in the widths of rings, since there are many instances of extended (apparently smooth) cores, bands, or belts of emission that strain the simplistic morphological definition. There is also considerable variety in the azimuthal extents of arcs, ranging from $\sim 5$ to $\gtrsim 100^{\circ}$ (e.g., Casassus et al. 2013; Tsukagoshi et al. 2019b).

Limited resolution also makes it challenging to quantify substructure contrasts. If the features are not well resolved, peaks could be higher and troughs could be lower than they appear: contrasts should be considered lower bounds. Moreover, mm continuum ring intensities will saturate at high optical depths: in that scenario, inferences of $\Sigma_{\mathrm{s}}$ contrasts could be much higher than the $I_{\nu}$ contrasts imply. For the well-resolved ring/gap pairs, contrasts range from a few percent to a factor of $\sim 100$ (Huang et al. 2018b; Long et al. 2018b; Avenhaus et al. 2018). Contrasts inferred for ring/cavity or arc substructures can be even higher (Andrews et al. 2011; van der Marel et al. 2013; Pinilla et al. 2018b). The contrasts between spirals and the local inter-arm material depends strongly on the tracer: while it can be high in scattered light (e.g., Benisty et al. 2015; Stolker et al. 2016), it is $\lesssim 3$ in the few examples available for the mm continuum (Huang et al. 2018c).

There are fewer quantitative constraints available for the substructure properties based on the intensity distributions of key gas tracers. In the ring/cavity cases, the gas is found to extend to smaller radii than the $\mathrm{mm} / \mathrm{cm}$ solids, although it ultimately is depleted to comparable levels (van der Marel et al. 2015b, 2016b). In some cases, spectral line measurements demonstrate that additional rings/gaps continue at distances well beyond the continuum emission (Huang et al. 2018a; Guzmán et al. 2018). Some preliminary analyses indicate that spectral line depletions (gaps) track their continuum counterparts (e.g., Isella et al. 2016, 2018). But generally, the relative lack of emission line constraints is largely a technical limitation, since such observations at very high resolution are considerably more expensive than for the mm continuum or scattered light. Such measurements are of high value, and should play increasingly important roles in future work.

5.3.3. Optical Depth Fine-Tuning. One subtle, puzzling outcome from the suite of new high resolution mm continuum observations potentially has wide-reaching implications. Presuming that continuum emission is optically thin, and adopting a simple prescription for $T(r)$, 
the observed intensities at most substructure peaks imply $\tau_{\nu} \approx 0.5$ (within a factor of two) at $\lambda=1.3 \mathrm{~mm}$ (Huang et al. 2018b; Dullemond et al. 2018). That value is suspiciously similar to the $\left\langle\tau_{\nu}\right\rangle$ estimated from the shape of the $R_{\mathrm{mm}}-L_{\mathrm{mm}}$ relation (Section 2.2; Tripathi et al. 2017). This fine-tuning seems artificial, as if it points to some important underlying process or flawed assumption. So far, three ideas have been proposed to explain it.

The first idea is a geometric argument: it presumes the emission is optically thick, but that the true brightness distribution is concentrated on sub-resolution scales (Tripathi et al. 2017; Andrews et al. 2018a). The second idea is a radiative transfer argument: it again presumes the emission is optically thick, but adds that self-scattering for particles with very high albedos $\left(\omega_{\nu} \gtrsim 0.9\right)$ is important (Zhu et al. 2019). In that case, scattering diminishes the intensities $I_{\nu}$, making the emission appear (marginally) optically thin even if the true $\tau_{\nu}$ is arbitrarily high. That said, the high $\omega_{\nu}$ requirement maps onto a narrow $a_{\text {max }}$ range $(\approx \lambda / 2 \pi)$ : it is worth considering the plausibility of populating the $\tau_{\nu} \sim 1$ layers of disks with a very specific size distribution, or if this is just a change of variable for the fine-tuning problem (from $\tau_{\nu}$ to $a_{\max }$ at a special altitude). The third idea is a physical argument: it takes the marginally thin optical depths at face value and considers a self-regulating exchange between $\kappa_{\nu}$ and $\Sigma_{\mathrm{s}}$ in local pressure maxima (Stammler et al. 2019). In this case, particle evolution simulations can explain the data if a fraction $(\sim 10 \%)$ of the solid mass is converted into planetesimals whenever the solids-to-gas ratio approaches unity.

While these ideas are fleshed out, it is important to evaluate more generally the implications of the two optically thick hypotheses. High optical depths are natural explanations for the $R_{\mathrm{mm}}-L_{\mathrm{mm}}$ relation, as well as the low $\alpha_{\mathrm{mm}}$ (Zhu et al. 2019; Liu 2019) or $\varepsilon$ in the inner disk regions (Figure 9) and substructure rings (Carrasco-González et al. 2016, 2019; Tsukagoshi et al. 2016; Huang et al. 2018a). However, this would force a re-evaluation of traditional estimates of disk masses and densities (Section 2), shift the interpretations of various demographic trends (Section 3), and perhaps severely complicate the analyses of spectral line observations (e.g., Weaver et al. 2018). Given the scope of what is at stake, identifying the origins of this fine-tuning puzzle is certainly a high priority.

5.3.4. Sample Bias and Resolution Limitations. The current sample of very high resolution measurements in the mm continuum or scattered light is biased in favor of larger, brighter disks that preferentially orbit more massive host stars (Andrews et al. 2018b; Garufi et al. 2018). This is a practical restriction, but also in some sense by design. To find and measure substructures with characteristic sizes $\approx H_{p}$ at the resolutions (and inner working angles) available from current facilities requires them to be located at $r \gtrsim 20-50$ au. A more representative target, around a host with half the mass and 5-10× lower $L_{\mathrm{mm}}$, would typically have its tracer emission concentrated within that critical radius. If the substructures closer to the stellar hosts are like those we find at larger $r$ (i.e., widths $\lesssim H_{p}$ ), these smaller disks will appear smooth even if they are also riddled with substructures.

Indeed, there is plenty of evidence that the distribution of continuum substructure sizes extends below $H_{p}$-scales and current resolution limits (e.g., from the many partially resolved features mentioned by Huang et al. 2018b). The advantageous distance of the TW Hya disk offers an instructive example: nearly all of its rings and gaps are narrow enough that they would be indistinguishable from an unperturbed emission profile if the system were moved out to the nearest young clusters (see Andrews et al. 2016; Huang et al. 2018a). This is not to say that efforts to find especially large substructures in more representative samples are undesirable: rather, the point is that any conclusions about the prevalence of substructures 
need to be contextualized to the accessible range of substructure sizes.

5.3.5. Kinematics. Another approach to quantifying substructures in $P(r)$ involves a search for the associated deviations in the radial velocity profile of the gas, $v_{\theta}$. These non-Keplerian motions can be identified when the residual velocity profile, $\delta v_{\theta}=\left(v_{\theta}-v_{\text {kep }}\right) / v_{\text {kep }}$, flips sign over a narrow spatial range: that signal can then be related to the local pressure gradient (Section 5.1). Observational constraints on the spatial patterns of $\delta v_{\theta}$ offer independent, kinematic insights on substructures that complement their intensity characterizations based on high resolution spectral line or continuum images.

With sufficient resolution and sensitivity, (sub-)mm spectral line datasets can be used to reconstruct azimuthally-averaged $\delta v_{\theta}(r)$ profiles in the line photosphere layers (Teague et al. 2018a,c; Pérez et al. 2018a). That technique has been used to identify kinematic perturbations with $\sim 5-10 \%$ amplitudes (at roughly percent level precision) that spatially coincide with known substructures in the mm continuum. Naturally, the azimuthal averaging required to tease out small $\delta v_{\theta}$ signals means that it is unclear whether or not those kinematic deviations are axisymmetric. If the perturbations are tracing the spiral wakes expected from planet-disk interactions, the $\delta v_{\theta}$ signal would peak in the immediate vicinity of the perturber (Pérez et al. 2015b, 2018a; Kanagawa et al. 2015; Teague et al. 2018a; Zhang et al. 2018). Especially strong localized $\delta v_{\theta}$ sign-flips have been identified in a few cases (Pinte et al. 2018b, 2019; Casassus \& Pérez 2019), lending striking support to such a dynamical origin even if the perturber itself cannot yet be directly detected.

Such kinematics constraints on disk properties are still in a relatively early stage of development, both observationally and theoretically. Nevertheless, they hold immense promise in their synergy with the more traditional measurements emphasized throughout this review. Leveraging these techniques together will be a necessary step in the push toward a more quantitative characterization of substructure properties.

5.3.6. Vertical Perturbations. The discussion above emphasizes the $(r, \theta)$-plane, but there are also clear signs of substructures in the vertical $(z)$ dimension. These can be inferred indirectly from infrared variability (Muzerolle et al. 2009; Flaherty et al. 2012; Rebull et al. 2014), especially in cases where the variations follow a "see-saw" spectral pattern with enhancements at shorter $\lambda$ (lower, warmer $r$ ) accompanied by depletions at longer $\lambda$ (higher, cooler $r$ ), and vice versa (Espaillat et al. 2011). These time-domain phenomena are presumably associated with the stellar obscuration and disk shadowing that occur when the vertical distribution of inner disk material is perturbed (e.g., Turner et al. 2010). In some cases, that shadowing can be seen directly in scattered light images (Garufi et al. 2014; Benisty et al. 2018), as illustrated in Figure 12. This obscuration can be quite variable on short timescales, indicating a clumpy distribution of occulting material in the inner disk (e.g., Stolker et al. 2017; Pinilla et al. 2018c). Debes et al. (2017) discovered an especially compelling example, where an outer disk shadow moves at a rate consistent with inner disk orbital timescales. There is a hypothesis that spirals identified in scattered light could be associated with such behavior (Kama et al. 2016a; Montesinos et al. 2016).

In most examples, shadows require a persistent vertical substructure in the inner disk. This is often associated with a warp, since even modest changes in the orbital inclination distribution of the disk material generate pronounced observational effects (Nealon et al. 2019). Warped geometries have been inferred kinematically with resolved spectral line data, based on the spatial variation of the projected (line-of-sight) velocities (Rosenfeld et al. 

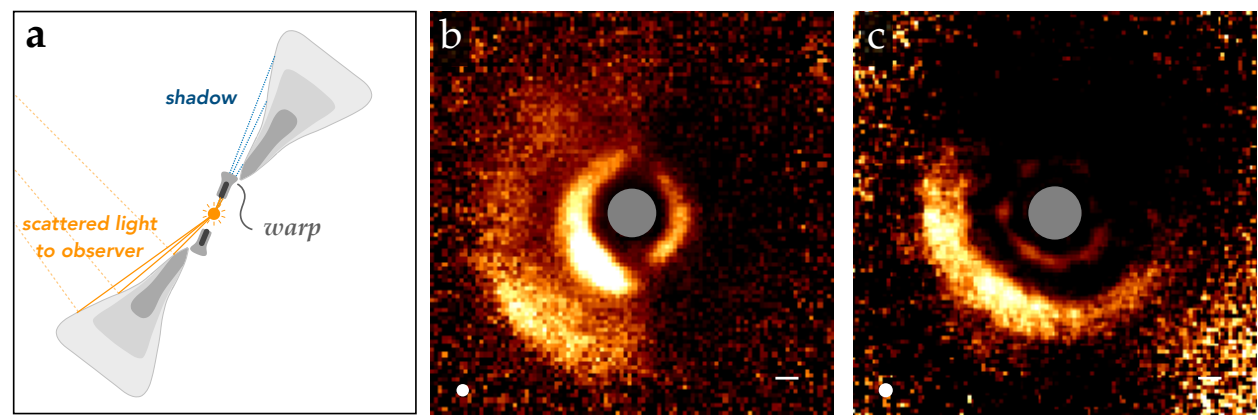

Figure 12

(a) A schematic illustration of how a disk warp can induce shadowing in scattered light images (see also Marino et al. 2015). (b) Two examples of shadowing from large-scale asymmetries in infrared scattered light images, from the disks around (b) HD 143006 (Benisty et al. 2018) and (c) WRAY 15- 788 (Bohn et al. 2019). Image annotations are as in Figure 11. A stronger warp, or even a "broken" disk geometry can change the azimuthal extent of the shadows (e.g., see the narrow shadows for the HD 100453 disk, at bottom right in Figure 11; Benisty et al. 2017).

2012b, 2014; Casassus et al. 2015a; Loomis et al. 2017). Large warps or misalignments ("broken" disks) can also induce scattered light shadows at larger $r$; the locations and azimuthal extents of the shadows help constrain the inner disk geometry (Marino et al. 2015; Stolker et al. 2016; Facchini et al. 2018; Pinilla et al. 2018c; Casassus et al. 2018).

In any case, a variety of vertical substructures are inferred at locations (and with sizes) that are well below current resolution capabilities. Nevertheless, their effects are manifested on much larger scales in the $(r, \theta)$ behavior of key tracers. The important lesson is that current observations are sensitive to substructures in all three spatial dimensions.

\subsection{Emerging Insights}

The specific topic of disk substructures has generated immense interest. The many new opportunities for high resolution observations of disks have triggered a marked pivot in the field toward their interpretation. Despite the deluge in the literature, it is worth keeping in mind that assessments of the broader impacts that substructures have on disk evolution and planet formation are still being actively developed. Nevertheless, it is also clear from their prevalence alone that substructures are fundamental aspects of disks: they likely have profound effects on every practical and physical facet of planet formation research.

The current priority is to develop a more quantitative understanding of the physical mechanism(s) responsible for generating substructures and how they impact (or perhaps reveal ongoing) planet formation. At this point, it is fair to conclude that none of the potential origins discussed in Section 5.2 can be categorically excluded. Without rehashing a detailed comparison of the data and model predictions, there are a few generic points about the emerging themes of this analysis that are worth highlighting.

One important conclusion is that the simple empirical signatures expected from models of particle migration around snowlines (Section 5.2.3) are not observed (van Terwisga et al. 2018; Long et al. 2018b; Huang et al. 2018b; van der Marel et al. 2019). Those signatures include a rough $L_{*}^{0.5}$ scaling to the pattern of ring/gap locations in the mm con- 

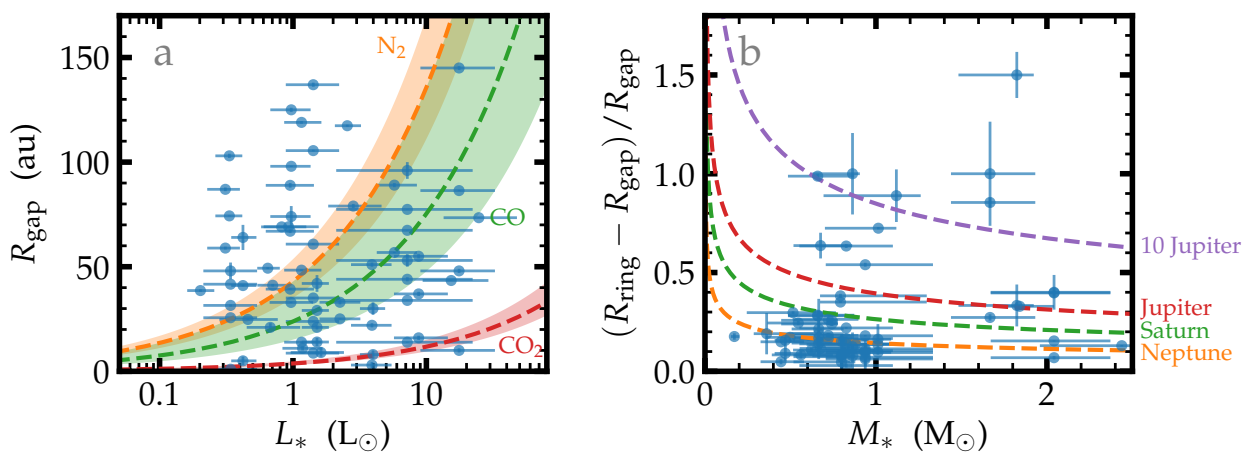

Figure 13

(a) The locations of gap substructures as a function of $L_{*}$ (data from Huang et al. 2018b; Long et al. 2018b). The overlaid shaded regions mark the expected locations for the condensation fronts of abundant volatiles (as labeled, following Huang et al. 2018b). There is no clear pattern indicating a connection between substructure locations and special disk temperatures. (b) The fractional separations between ring and gap substructure pairs (from these same studies) as a function of $M_{*}$. The overlaid curves mark the masses of planets that might be responsible for opening the gaps, following the simplified assumptions of Long et al. (2018b) and Lodato et al. (2019). The implied masses (and orbits, examining panel a) probe a very different range of parameter-space from the mature exoplanet population (see also Zhang et al. 2018).

tinuum and an $r^{-0.5}$ spacing between them, and originate from the hypothesis that such substructures occur at special locations corresponding to the condensation temperatures of abundant volatiles. Figure 13a demonstrates that these patterns are not obvious in the data. However, there is considerable diversity in the model predictions, and one cannot rule out that any individual feature (or the collection of features in individual disks) might be associated with snowlines (Zhang et al. 2015; van der Marel et al. 2018a). More extensive vetting of this hypothesis will consider the non-trivial uncertainties in the disk temperatures (Section 2.4) and the dependence of condensation temperatures on the bulk ice composition and local gas pressure. Meanwhile, some theoretical consensus would be useful: recent models predict either emission enhancements or depletions, located either inside or outside the snowline (Section 5.2.3). It would help to know whether or not that fungible range of outcomes represents an inherent physical ambiguity.

There are additional insights that disfavor the snowline hypothesis, in that there is quantitative evidence that many observed substructures do trace particle traps at local gas pressure maxima. The subset of resolved $\mathrm{mm}$ continuum rings are found to have the high amplitudes and narrow widths $\left(<H_{p}\right)$ predicted for these traps (Dullemond et al. 2018). Complementary support for that conclusion is also available from the demographics (Pinilla et al. 2018b), kinematics (Teague et al. 2018a,c), diverse tracer-dependent morphologies in ring/cavity substructures (Dong et al. 2012; van der Marel et al. 2015b), and narrow azimuthal extents of continuum emission with low $\varepsilon$ at the peaks of arc substructures (Birnstiel et al. 2013; van der Marel et al. 2015a; Casassus et al. 2015b). These constraints lend credibility to the fluid mechanics or planet-disk interactions hypotheses for substructure origins, although robustly discriminating between those options is perhaps not yet practical (Flock et al. 2015; Ruge et al. 2016; Dong et al. 2018).

That said, the mechanism(s) that generate these very detailed disk substructures really 
color the global perspective on what information can be gleaned from disk properties. If fluid perturbations from various (M)HD processes are ultimately responsible, then disks are genuinely in a classical "protoplanetary" phase, representative of incipient planet formation. Detailed measurements of their properties would illustrate how disk substructures are fundamental for making planetesimals. Much of the immediate progress to be made in testing this hypothesis will come from enhanced computational capabilities, to help develop more robust and discriminating predictions. The alternative hypothesis, that substructures are instead produced by perturbations from already-formed planetary systems, has subtle but profound implications for the standard principles of planet formation theories.

To discuss those implications, it helps to consider the masses and orbits of the youthful planetary systems that are inferred from the morphologies of the disk substructures. With reference to simulations of disk-planet interactions, the locations, widths, and depths of disk gaps (Zhang et al. 2018; Lodato et al. 2019) and cavities (e.g., Zhu et al. 2012) suggest perturber masses from a few $\mathrm{M}_{\oplus}$ to $\sim 10 \mathrm{M}_{\text {Jup }}$ orbiting at semimajor axes of $\sim 10-150$ au (around a representative $\sim$ Sun-like host). Figure $13 \mathbf{b}$ illustrates some representative results. Note that this is a different (complementary) region of parameter-space for planetary system architectures than has been probed in exoplanet surveys around mature host stars (early direct imaging constraints overlap at the high-mass end). The timescales to form such planets in the standard (core accretion) formation theory are considerably longer than the typical system ages $(\sim 1-3 \mathrm{Myr})$. So, if such planets already exist, they require that the formation process starts very early - perhaps overlapping with the epoch of star (and disk) formation itself - or is substantially accelerated (e.g., perhaps with some variant of pebble accretion; Ormel \& Klahr 2010; Lambrechts \& Johansen 2012). In either case planetesimal formation must be efficient and prolific over a wide range of disk radii, presumably aided by an earlier generation of substructures (perhaps generated by an assortment of fluid dynamical mechanisms that are more prevalent during the embedded phase).

The planet-disk interaction hypothesis is certainly an exciting prospect, since it offers potential opportunities to observationally constrain planet formation timescales, planetary accretion and satellite formation (through studies of circumplanetary material), and the evolution of planetary system architectures (i.e., planetary migration). And there is increasing confidence in this option, especially from observations of strong gas depletion in disk cavities (van der Marel et al. 2015b, 2016b), spatially isolated perturbations to the disk gas dynamics that coincide with substructures (Casassus \& Pérez 2019; Pinte et al. 2019), and most importantly the direct imaging detections of young giant planets in the cavity of the PDS 70 disk (Keppler et al. 2018; Müller et al. 2018; Haffert et al. 2019). Nevertheless, there is still much work to do regarding the origins of disk substructures: assessing these forking paths is the single most important task of the coming decade in this field.

\section{SYNOPSIS \& OUTLOOK}

\section{SUMMARY POINTS}

1. New quantitative insights on key structure parameters are starting to bear fruit, but the intrinsic uncertainties on physical conditions suggest it is important to consider empirical metrics and translate predictions into the data-space. 
2. There is clear evidence for multi-dimensional demographic relationships between disk properties (mm continuum luminosities, sizes) and various dynamical $\left(M_{*}\right)$, environmental (e.g., multiplicity), and evolutionary factors.

3. While observations provide strong, qualitative support for the predicted behaviors of particle growth and migration models (particularly the spatial segregation of particle sizes and the radial gradient in the solids-to-gas ratio), there is a timescale discrepancy that suggests a smooth gas pressure profile is a poor assumption.

4. Small-scale substructures with a range of morphologies are found on scales comparable to $H_{p}$ throughout many (and perhaps all) disks, with dimensions and contrasts consistent with expectations for particle trapping in local gas pressure maxima.

5. The origins of these substructures are not yet clear: leading contenders include an assortment of fluid instabilities or dynamical interactions with young planets. In any case, the implications are profound, in that (physical and observational) disk properties could be determined by perturbations at very small scales.

\section{FUTURE ISSUES}

1. Quantitative constraints on the density and temperature structure of the gas in disks are essential to enable progress in the field. Investments in deep, high resolution observations of molecular spectral line emission should be prioritized.

2. The velocity dimension of those spectral line datasets is a rich frontier for reaping physical information. Kinematic studies of turbulent motions and non-Keplerian deviations to the velocity field are expected to create many new opportunities to address theoretical predictions from a complementary perspective.

3. There is vast potential to develop a better understanding of disk properties encoded in their demographic relationships (and associated scatter). Expanding that work to more diverse samples (e.g., ages, multiplicity parameters) and additional metrics (e.g., deeper, resolved spectral line data) would be highly valuable.

4. Progress on quantifying the evolution of disk solids will require a continued pursuit of spatial variations in the $\mathrm{mm} / \mathrm{cm}$ continuum spectrum and polarization properties. Folding those properties into demographics studies would be especially illuminating.

5. A shift to quantitative characterizations of disk substructures - density contrasts, diffusion, particle sizes, kinematics, or their empirical equivalents - would provide welcome guidance for theoretical predictions and help better assess their origins.

6. An appraisal of how the prevalence, morphologies, locations, and scales of disk substructures depend on host properties, global disk characteristics, environment, and especially age could reveal patterns that help contextualize general demographic trends and clarify the mechanics and variety of the processes that generate them.

\section{DISCLOSURE STATEMENT}

The author is not aware of any affiliations, memberships, funding, or financial holdings that might be perceived as affecting the objectivity of this review. 


\section{ACKNOWLEDGMENTS}

I am very grateful to Myriam Benisty, Til Birnstiel, Marco Tazzari, and David Wilner for their detailed counsel and assistance with some of the ideas and figures in this review. Xuening Bai, John Carpenter, Kees Dullemond, Michiel Hogerheijde, Feng Long, Antonella Natta, Karin Öberg, Paola Pinilla, Richard Teague, Nienke van der Marel, Ewine van Dishoeck, Jonathan Williams, and Zhaohuan Zhu provided helpful, detailed comments on drafts that helped to substantially improve the writing, organization, and general presentation of ideas. Juan Alcalá, Jane Huang, Meredith Hughes, Kevin Flaherty, and James Owen offered useful advice that helped crystallize ideas and writing strategies. I also want to thank Megan Ansdell, Paolo Cazzoletti, Lucas Cieza, Ian Czekala, Ruobing Dong, Nathan Hendler, Stefan Kraus, Tomoyuki Kudo, Ryan Loomis, Sebastian Pérez, Dary RuizRodriguez, Pat Sheehan, Roy van Boekel, Gerrit van der Plas, and Sierk van Terwisga, in addition to some of those listed above, for kindly sharing their data. This review made extensive use of the numpy (van der Walt et al. 2011), matplotlib (Hunter 2007), astropy (Astropy Collaboration et al. 2018), and dsharp-opac (Birnstiel et al. 2018) software packages, as well as NASA's Astrophysics Data System and the SIMBAD database, operated at CDS, Strasbourg, France. 


\section{LITERATURE CITED}

Aikawa Y, Umebayashi T, Nakano T, Miyama SM. 1997. Ap. J. Lett. 486:L51 [2.1]

Akeson RL, Jensen ELN. 2014. Ap. J. 784:62 [3.2.1]

Akeson RL, Jensen ELN, Carpenter J, et al. 2019. Ap. J. 872:158 [3, 3.1, 3.2.1]

Akiyama E, Hashimoto J, Liu HB, et al. 2016. Astron. J. 152:222 [5.3.1]

Akiyama E, Muto T, Kusakabe N, et al. 2015. Ap. J. Lett. 802:L17 [5.3.1]

Alexander R, Pascucci I, Andrews S, Armitage P, Cieza L. 2014. In Protostars and Planets VI, ed. H Beuther, RS Klessen, CP Dullemond, T Henning, pp. 475-496. Tucson: Univ. Ariz. Press $[3.2 .2,5.2 .1]$

ALMA Partnership, Brogan CL, Pérez LM, et al. 2015. Ap. J. Lett. 808:L3 [5.3.1, 11]

Alves FO, Caselli P, Girart JM, et al. 2019. Science 366:90-93 [3.2.1]

Andersen BC, Stephens IW, Dunham MM, et al. 2019. Ap. J. 873:54 [3.3]

Andrews SM, Rosenfeld KA, Kraus AL, Wilner DJ. 2013. Ap. J. 771:129 [3, 3.1, 3.1]

Andrews SM, Williams JP. 2005. Ap. J. 631:1134-1160 [2.1, 4.3.2]

-. 2007a. Ap. J. 659:705-728 [2.3, 4.3.2]

-. 2007b. Ap. J. 671:1800-1812 [3.3]

Andrews SM, Wilner DJ, Hughes AM, Qi C, Dullemond CP. 2009. Ap. J. 700:1502-1523 [2.3]

-. 2010. Ap. J. 723:1241-1254 [2.2, 2.3]

Andrews SM, Huang J, Pérez LM, et al. 2018b. Ap. J. Lett. 869:L41 [6, 5.3.1, 11, 5.3.4]

Andrews SM, Terrell M, Tripathi A, et al. 2018a. Ap. J. 865:157 [2.2, 4, 3.1, 5.3.3]

Andrews SM, Wilner DJ, Espaillat C, et al. 2011. Ap. J. 732:42 [5.3.1, 5.3.2]

Andrews SM, Wilner DJ, Hughes AM, et al. 2012. Ap. J. 744:162 [2.2, 4.3.5]

Andrews SM, Wilner DJ, Zhu Z, et al. 2016. Ap. J. Lett. 820:L40 [2, 5.3.1, 5.3.4]

Ansdell M, Williams JP, van der Marel N, et al. 2016. Ap. J. 828:46 [3, 2.1, 3.1]

Ansdell M, Williams JP, Manara CF, et al. 2017. Astron. J. 153:240 [3.2.2]

Ansdell M, Williams JP, Trapman L, et al. 2018. Ap. J. 859:21 [3, 2.1, 4, 2.2, 4.3.2]

Armitage PJ. 2011. Annu. Rev. Astron. Astrophys. 49:195-236 [2.5]

Artymowicz P, Lubow SH. 1994. Ap. J. 421:651-667 [3.2.1]

Astropy Collaboration, Price-Whelan AM, Sipőcz BM, et al. 2018. Astron. J. 156:123 [6]

Ataiee S, Pinilla P, Zsom A, et al. 2013. Astron. Astrophys. 553:L3 [5.2.2]

Avenhaus H, Quanz SP, Garufi A, et al. 2018. Ap. J. 863:44 [5.3.1, 11, 5.3.2]

Avenhaus H, Quanz SP, Schmid HM, et al. 2014. Ap. J. 781:87 [5.3.1, 11]

Bacciotti F, Girart JM, Padovani M, et al. 2018. Ap. J. Lett. 865:L12 [4.3.3]

Bae J, Hartmann L, Zhu Z. 2015. Ap. J. 805:15 [5.2.1]

Bae J, Zhu Z. 2018. Ap. J. 859:118 [5.2.2]

Bae J, Zhu Z, Hartmann L. 2017. Ap. J. 850:201 [5.2.2]

Bai X-N, Stone JM. 2013. Ap. J. 769:76 [2.3, 2.5]

—. 2014. Ap. J. 796:31 [5.2.1]

Balbus SA, Hawley JF. 1991. Ap. J. 376:214-222 [2.5]

Barenfeld SA, Carpenter JM, Ricci L, Isella A. 2017a. Ap. J. 827:142 [3, 3.1, 3.3]

Barenfeld SA, Carpenter JM, Sargent AI, Isella A, \& Ricci L. 2017b. Ap. J. 851:85 [2.2, 3.3]

Barge P, Sommeria J. 1995. Astron. Astrophys. 295:L1-L4 [5.2.1]

Baruteau C, Crida A, Paardekooper S-J, et al. 2014. In Protostars and Planets VI, ed. H Beuther, RS Klessen, CP Dullemond, T Henning, pp. 667-689. Tucson: Univ. Ariz. Press [2.3]

Baruteau C, Zhu Z. 2016. MNRAS 458:3927-3941 [5.2.1]

Bate MR. 2018. MNRAS 475:5618-5658 [3.2.1]

Beckwith SVW, Sargent AI, Chini RS, Guesten R. 1990. Astron. J. 99:924-945 [2.1]

Beckwith SVW, Sargent AI. 1991. Ap. J. 381:250-258 [4.3.2]

—. 1993. Ap. J. 402:280-291 [2.1]

Bell CPM, Naylor T, Mayne NJ, Jeffries RD, Littlefair SP. 2013. MNRAS 434:806-831 [3.3]

Benisty M, Juhasz A, Boccaletti A, et al. 2015. Astron. Astrophys. 578:L6 [11, 5.3.1, 5.3.2] 
Benisty M, Stolker T, Pohl A, et al. 2017. Astron. Astrophys. 597:A42 [11, 12]

Benisty M, Juhász A, Facchini S, et al. 2018. Astron. Astrophys. 619:A171 [5.3.6, 12]

Benz W, Ida S, Alibert Y, et al. 2014. In Protostars and Planets VI, ed. H Beuther, RS Klessen,

CP Dullemond, T Henning, pp. 691-713. Tucson: Univ. Ariz. Press [1.1]

Bergin EA, Cleeves LI, Gorti U, et al. 2013. Nature 493:644-646 [2.1]

Bertrang GHM, Flock M, Wolf S. 2017. MNRAS 464:L61-L64 [2.5]

Bertrang GHM, Wolf S. 2017. MNRAS 469:2869-2878 [4.1]

Béthune W, Lesur G, \& Ferreira J. 2016. Astron. Astrophys. 589:A87 [5.2.1]

Birnstiel T, Dullemond CP, Brauer F. 2009. Astron. Astrophys. 503:L5-L8 [4.1]

-. 2010. Astron. Astrophys. 513:A79 [4.1, 4.4, 5.2.3]

Birnstiel T, Ormel CW, Dullemond CP. 2011. Astron. Astrophys. 525:A11 [4.1, 4.2]

Birnstiel T, Klahr H, Ercolano B. 2012, Astron. Astrophys. 539:A148 [2.3]

Birnstiel T, Dullemond CP, Pinilla P. 2013. Astron. Astrophys. 550:L8 [5.4]

Birnstiel T, Andrews SM. 2014. Ap. J. 780:153 [4.1]

Birnstiel T, Andrews SM, Pinilla P, Kama M. 2015. Ap. J. Lett. 813:L14 [4.1]

Birnstiel T, Dullemond CP, Zhu Z, et al. 2018. Ap. J. Lett. 869:L45 [4.1, 8, 4.2, 6]

Blandford RD, Payne, DG. 1982. MNRAS 199:883-903 [2.3]

Boehler Y, Dutrey A, Guilloteau S, Piétu V. 2013. MNRAS 431:1573-1586 [4.3.1]

Boehler Y, Ricci L, Weaver E, et al. 2018. Ap. J. 853:162 [5.3.1]

Bohn AJ, Kenworthy MA, Ginski C, et al. 2019. Astron. Astrophys. 624:A87 [12]

Booth AS, Walsh C, Ilee JD, et al. 2019. Ap. J. Lett. 882:L31 [2.1]

Bosman AD, Walsh C, van Dishoeck E. 2018. Astron. Astrophys. 618:A182 [2.1]

Boss, AP. 1997. Science 276:1836-1839 [5.2.1]

Boudet N, Mutschke H, Nayral C, et al. 2005. Ap. J. 633:272-281 [4.1]

Brauer F, Dullemond CP, Johansen A, et al. 2007. Astron. Astrophys. 469:1169-1182 [4.1]

Brauer F, Dullemond CP, Henning T. 2008. Astron. Astrophys. 480:859-877 [4.1]

Brauer R, Wolf S, Flock M. 2017. Astron. Astrophys. 607:A104 [2.5]

Breslau A, Steinhausen M, Vincke K, Pfalzner S. 2014. Astron. Astrophys. 565:A130 [3.2.1]

Brinch C, Jørgensen JK, Hogerheijde MR, Nelson RP, Gressel O. 2016. Ap. J. Lett. 830:L16 [3.2.1]

Brown JM, Blake GA, Qi C, et al. 2009. Ap. J. 704:496-502 [5.3.1]

Bruderer S. 2013. Astron. Astrophys. 559:A46 [2.4]

Calvet N, Patino A, Magris GC, D'Alessio P. 1991. Ap. J. 380:617-630 [2.4]

Calvet N, D'Alessio P, Hartmann L, et al. 2002. Ap. J. 568:1008-1016 [5.3.1]

Carrasco-González C, Henning T, Chandler CJ, et al. 2016. Ap. J. Lett. 821:L16 [5.3.3]

Carrasco-González C, Sierra A, Flock M, et al. 2019. Ap. J. 883:71 [5.3.3]

Casassus S, van der Plas G, Pérez S, et al. 2013. Nature 493:191-194 [5.3.1, 11, 5.3.2]

Casassus S, Marino S, Pérez S, et al. 2015a. Ap. J. 811:92 [5.3.6]

Casassus S, Wright CM, Marino S, et al. 2015b. Ap. J. 812:126 [5.4]

Casassus S, Avenhaus H, Pérez S, et al. 2018. MNRAS 477:5104-5114 [5.3.6]

Casassus S, Pérez S. 2019. Ap. J. Lett. 883:L41 [5.3.5, 5.4]

Cazzoletti P, van Dishoeck EF, Pinilla P, et al. 2018. Astron. Astrophys. 619:A161 [11, 5.3.1]

Chiang EI, Goldreich P. 1997. Ap. J. 490:368-376 [2.4]

Chiang E, Laughlin G. 2013. MNRAS 431:3444-3455 [2.1]

Cho J, Lazarian A. 2007. Ap. J. 669:1085-1097 [2.5]

Christiaens V, Casassus S, Pérez S, van der Plas G, Ménard F. 2014. Ap. J. Lett. 785:L12 [5.3.1]

Ciesla FJ, Cuzzi JN. 2006. Icarus 181:178-204 [5.2.3]

Cieza L, Williams J, Kourkchi E, et al. 2015. MNRAS 454:1909-1920 [9]

Cieza LA, Casassus S, Tobin J, et al. 2016. Nature 535:258-261 [5.3.1]

Cieza LA, Ruíz-Rodríguez D, Hales A, et al. 2019. MNRAS 482:698-714 [3]

Clarke CJ, Pringle JE. 1993. MNRAS 261:190-202 [3.2.1]

Clarke CJ, Tazzari M, Juhász A, et al. 2018. Ap. J. Lett. 866:L6 [5.3.1] 
Cleeves LI, Adams FC, Bergin EA, Visser R. 2013. Ap. J. 777:28 [2.4] Cleeves LI, Bergin EA, Öberg KI, et al. 2017. Ap. J. Lett. 843:L3 [2.3] Cox EG, Harris RJ, Looney LW, et al. 2017. Ap. J. 851:83 [9]

Cuello N, Dipierro G, Mentiplay D, et al. 2019. MNRAS 483:4114-4139 [3.2.1]

Cuzzi JN, Hogan RC, Paque JM, et al. 2001. Ap. J. 546:496-508 [5.2.1]

Cuzzi JN, Zahnle KJ. 2004. Ap. J. 614:490-496 [5.2.3]

Cuzzi JN, Estrada PR, Davis, SS. 2014. Ap. J. Suppl. 210:21 [4.1]

Czekala I, Andrews SM, Jensen ELN, et al. 2015. Ap. J. 806:154 [2.5]

D'Alessio P, Calvet N, Hartmann L. 1997. Ap. J. 474:397-406 [2.4]

—. 2001. Ap. J. 553:321-334 []

D'Alessio P, Cantó J, Calvet N, Lizano S. 1998. Ap. J. 500:411-427 [2.4]

D'Alessio P, Calvet N, Hartmann L, Lizano S, Cantó J. 1999. Ap. J. 527:893-909 [2.4]

D’Alessio P, Calvet N, Hartmann L, Franco-Hernández R, Servín H. 2006. Ap. J. 638:314-335 [2.4, 4.1, 4.3.1]

Dartois E, Dutrey A, Guilloteau S. 2003. Astron. Astrophys. 399:773-787 [2.3, 2.4]

de Boer J, Salter G, Benisty M, et al. 2016. Astron. Astrophys. 595:A114 [5.3.1]

de Juan Ovelar M, Kruijssen JMD, Bressert E, et al. 2012. Astron. Astrophys. 546:L1 [3.2.1]

Debes JH, Poteet CA, Jang-Condell H, et al. 2017. Ap. J. 835:205 [5.3.6]

Dent WRF, Pinte C, Cortes PC, et al. 2019. MNRAS 482:L29-L33 [4.3.3]

Dipierro G, Pinilla P, Lodato G, Testi L. 2015. MNRAS 451:974-986 [5.2.1]

Dipierro G, Laibe G, Price DJ, Lodato G. 2016. MNRAS 459:L1-L5 [5.2.2]

Dittrich K, Klahr H, Johansen A. 2013. Ap. J. 763:117 [5.2.1]

Dodson-Robinson SE, Salyk C. 2011. Ap. J. 738:131 [5.2.2]

Dohnanyi JS. 1969. Journal Geo. Research 74:2531-2554 [4.2]

Dominik C, Tielens AGGM. 1997. Ap. J. 480:647-673 [4.1]

Dong R, Rafikov R, Zhu Z, et al. 2012. Ap. J. 750:161 [5.3.1, 5.4]

Dong R, Zhu Z, Rafikov RR, Stone, JM. 2015. Ap. J. Lett. 809: L5 [5.2.2]

Dong R, Li S, Chiang E, Li H. 2017. Ap. J. 843:127 [5.2.2]

Dong R, Liu S-y, Eisner J, et al. 2018a. Ap. J. 860:124 [11, 5.3.1]

Dong R, Najita JR, Brittain S. 2018b. Ap. J. 862:103 [5.4]

Draine BT. 2006. Ap. J. 636:1114-1120 [4.2]

Drążkowska J, Alibert Y. 2017. Astron. Astrophys. 608:A92 [5.2.3]

Drążkowska J, Alibert Y, Moore B. 2016. Astron. Astrophys. 594:A105 [5.2.1]

Dubrulle B, Morfill G, Sterzik M. 1995. Icarus 114:237-246 [2.4, 4.1]

Duchêne G, McCabe C, Pinte C, et al. 2010. Ap. J. 712:112-129 [4.3.1]

Dullemond CP, Dominik C. 2004a. Astron. Astrophys. 417:159-168 [4.1]

-. 2004b. Astron. Astrophys. 421:1075-1086 [2.4, 4.3.1]

-. 2005. Astron. Astrophys. 434:971-986 [4.1]

Dullemond CP, Dominik C, Natta A. 2001. Ap. J. 560:957-969 [2.4]

Dullemond CP, Penzlin ABT. 2018. Astron. Astrophys. 609:A50 [5.2.1]

Dullemond CP, van Zadelhoff GJ, Natta A. 2002, Astron. Astrophys. 389:464-474 [2.4]

Dullemond CP, Birnstiel T, Huang J, et al. 2018. Ap. J. Lett. 869:L46 [2.5, 5.3, 5.3.2, 5.3.3, 5.4]

Dullemond CP, Isella A, Andrews SM, Skobleva I, Dzyurkevich N. 2020. Astron. Astrophys. in press, arXiv:1911.12434 [2.4]

Dutrey A, Guilloteau S, Simon M. 2003. Astron. Astrophys. 402:1003-1011 [2.1]

Dzyurkevich N, Turner NJ, Henning T, Kley W. 2013. Ap. J. 765:114 [5.2.1]

Eisner JA, Arce HG, Ballering NP, et al. 2018. Ap. J. 860:77 [3.2.2]

Ercolano B, Pascucci I. 2017. Royal Soc. Open Sci. 4:170114 [2.5, 5.2.1]

Espaillat C, Calvet N, D'Alessio P, et al. 2007. Ap. J. Lett. 670:L135-L138 [5.3.1]

Espaillat C, Furlan E, D'Alessio P, et al. 2011. Ap. J. 728:49 [5.3.6]

Espaillat C, Muzerolle J, Najita J, et al. 2014. In Protostars and Planets VI, ed. H Beuther, RS 
Klessen, CP Dullemond, T Henning, pp. 497-520. Tucson: Univ. Ariz. Press [5.3.1] Estrada PR, Cuzzi JN, Morgan DA. 2016. Ap. J. 818:200 [5.2.3]

Facchini S, Birnstiel T, Bruderer S, van Dishoeck EF. 2017. Astron. Astrophys. 605:A16 [4.3.5] Facchini S, Clarke CJ, Bisbas TG. 2016. MNRAS 457:3593-3610 [3.2.2]

Facchini S, Juhász A, Lodato G. 2018. MNRAS 473:4459-4475 [5.3.6]

Facchini S, van Dishoeck EF, Manara CF, et al. 2019. Astron. Astrophys. 626:L2 [4, 2.2]

Favre C, Cleeves LI, Bergin EA, Qi C, Blake GA. 2013. Ap. J. Lett. 776:L38 [2.1]

Finkbeiner DP, Davis M, Schlegel DJ. 1999. Ap. J. 524:867-886 [4.2]

Flaherty KM, Muzerolle J, Rieke G, et al. 2012. Ap. J. 748:71 [5.3.6]

Flaherty KM, Hughes AM, Rosenfeld KA, et al. 2015. Ap. J. 813:99 [2.5]

Flaherty KM, Hughes AM, Rose SC, et al. 2017. Ap. J. 843:150 [2.5]

Flaherty KM, Hughes AM, Teague R, et al. 2018. Ap. J. 856:117 [2.5]

Flaherty K, Hughes AM, Simon JB, et al. 2019. Ap. J. submitted []

Flock M, Dzyurkevich N, Klahr H, Turner NJ, Henning T. 2011. Ap. J. 735:122 [5.2.1]

Flock M, Ruge JP, Dzyurkevich N, et al. 2015. Astron. Astrophys. 574:A68 [5.4]

Follette KB, Grady CA, Swearingen JR, et al. 2015. Ap. J. 798:132 [11]

Fukagawa M, Tamura M, Itoh Y, et al. 2010. Pub. Ast. Soc. Japan 62:347 [4.3.1]

Furlan E, Luhman KL, Espaillat C, et al. 2011. Ap. J. Supp. 195:3 [4.3.1]

Gammie CF. 1996. Ap. J. 457:355-362 [5.2.1]

Gárate M, Birnstiel T, Drążkowska J, Stammler SM. 2019. arXiv e-prints arXiv:1906.07708 [5.2.3]

Garufi A, Quanz SP, Schmid HM, et al. 2014. Astron. Astrophys. 568:A40 [5.3.6]

-. 2016. Astron. Astrophys. 588:A8 [5.3.1]

Garufi A, Benisty M, Pinilla P, et al. 2018. Astron. Astrophys. 620:A94 [2.2, 5.3.1, 5.3.1, 5.3.4]

Ginski C, Stolker T, Pinilla P, et al. 2016. Astron. Astrophys. 595:A112 [5.3.1]

Goldreich P, Ward WR. 1973. Ap. J. 183:1051-1062 [5.1]

Goldreich P, Kylafis ND. 1981. Ap. J. Lett. 243:L75-L78 [2.5]

Goldreich P, Tremaine S. 1980. Ap. J. 241:425-441 [5.2.2]

Gonzalez JF, Laibe G, Maddison ST. 2017. MNRAS 467:1984-1996 [5.2.1]

Grady CA, Muto T, Hashimoto J, et al. 2013. Ap. J. 762:48 [5.3.1]

Greaves JS, Rice WKM. 2010. MNRAS 407:1981-1988 [2.1]

Guilloteau S, Dutrey A, Piétu V, Boehler Y. 2011. Astron. Astrophys. 529:A105 [4.3.2]

Guilloteau S, Dutrey A, Wakelam V, et al. 2012. Astron. Astrophys. 548:A70 [2.5]

Guilloteau S, Piétu V, Chapillon E, et al. 2016. Astron. Astrophys. 586:L1 [4.3.2]

Güttler C, Blum J, Zsom A, Ormel CW, Dullemond, CP. 2010. Astron. Astrophys. 513:A56 [4.1]

Guzmán VV, Huang J, Andrews SM, et al. 2018. Ap. J. Lett. 869:L48 [5.3.1, 11, 5.3.2]

Haffert SY, Bohn AJ, de Boer J, et al. 2019. Nature Astronomy 3:749-754 [5.4]

Haisch KE, Lada EA, Lada CJ. 2001. Ap. J. Lett. 553:L153-L156 [3.3]

Hall C, Dong R, Rice K, et al. 2019. Ap. J. 871:228 [5.2.1]

Harris RJ, Andrews SM, Wilner DJ, Kraus AL. 2012. Ap. J. 751:115 [3.2.1, 9]

Hartmann L, Calvet N, Gullbring E, D'Alessio P. 1998. Ap. J. 495:385-400 [2.3]

Hashimoto J, Tamura M, Muto T, et al. 2011. Ap. J. Lett. 729:L17 [5.3.1]

Heese S, Wolf S, Dutrey A, Guilloteau S. 2017. Astron. Astrophys. 604:A5 [2.4]

Heinemann T, Papaloizou JCB. 2009. MNRAS 397:64-74 [5.2.1]

Hendler N, Pascucci I, Pinilla P, et al. 2020. Ap. J., in press, arXiv:2001.02666 [2.2, 4, 3.3]

Henning T, Stognienko R. 1996. Astron. Astrophys. 311:291-303 [4.1, 4.2]

Hernández J, Hartmann L, Megeath T, et al. 2007. Ap. J. 662:1067-1081 [3.3]

Hollenbach D, Johnstone D, Lizano S, Shu F. 1994. Ap. J. 428:654-669 [3.2.2]

Huang J, Andrews SM, Cleeves LI, et al. 2018a. Ap. J. 852:122 [2, 5.3.2, 5.3.3, 5.3.4]

Huang J, Andrews SM, Dullemond CP, et al. 2018b. Ap. J. Lett. 869:L42 [5.3.1, 5.3.2, 5.3.3, 5.3.4, $5.4,13]$

Huang J, Andrews SM, Pérez LM, et al. 2018c. Ap. J. Lett. 869:L43 [5.3.1, 11, 5.3.2] 
Hughes AM, Wilner DJ, Qi C, Hogerheijde MR. 2008. Ap. J. 678:1119-1126 [2.2, 4.3.5]

Hughes AM, Wilner DJ, Cho J, et al. 2009a. Ap. J. 704:1204-1217 [4.3.3]

Hughes AM, Andrews SM, Espaillat C, et al. 2009b. Ap. J. 698:131-142 [5.3.1]

Hughes AM, Wilner DJ, Andrews SM, Qi C, Hogerheijde MR. 2011. Ap. J. 727:85 [2.5]

Hull CLH, Yang H, Li Z-Y, et al. 2018. Ap. J. 860:82 [4.3.3]

Hunter JD. 2007. Comp. in Sci. and Eng. 9:90 [6]

Isella A, Testi L, Natta A, et al. 2007. Astron. Astrophys. 469:213-222 [4.3.5]

Isella A, Carpenter JM, Sargent AI. 2009. Ap. J. 701:260-282 [2.2, 2.3]

—. 2010. Ap. J. 714:1746-1761 [4.3.2]

Isella A, Pérez LM, Carpenter JM, et al. 2013. Ap. J. 775:30 [5.3.1]

Isella A, Guidi G, Testi L, et al. 2016. Phys. Rev. Lett. 117:251101 [5.3.2]

Isella A, Huang J, Andrews SM, et al. 2018. Ap. J. Lett. 869:L49 [2.3, 5.3.1, 11, 5.3.2]

Jensen ELN, Akeson R. 2014. Nature 511:567-569 [3.2.1]

Jensen ELN, Mathieu RD, Fuller GA. 1994. Ap. J. Lett. 429:L29-L32 [3.2.1]

Johansen A, Oishi JS, Mac Low M-M, et al. 2007. Nature 448:1022-1025 [5.1]

Johansen A, Youdin A, Klahr H. 2009. Ap. J. 697:1269-1289 [2.3, 5.2.1]

Johnstone D, Hollenbach D, Bally J. 1998. Ap. J. 499:758-776 [3.2.2]

Jørgensen JK, van Dishoeck EF, Visser R, et al. 2009. Astron. Astrophys. 507:861-879 [3.3]

Juhász A, Rosotti GP. 2018. MNRAS 474:L32-L36 [5.3.2]

Kama M, Pinilla P, Heays AN. 2016a. Astron. Astrophys. 593:L20 [5.3.6]

Kama M, Bruderer S, Carney M, et al. 2016b. Astron. Astrophys. 588:A108 [2.1]

Kamp I, Dullemond CP. 2004. Ap. J. 615:991-999 [2.4]

Kanagawa KD, Tanaka H, Muto T, Tanigawa T, Takeuchi T. 2015. MNRAS 448:994-1006 [5.3.5]

Kataoka A, Okuzumi S, Tanaka H, Nomura H. 2014. Astron. Astrophys. 568:A42 [4.2]

Kataoka A, Muto T, Momose M, et al. 2015. Ap. J. 809:78 [2.5, 4.3.3]

Kataoka A, Muto T, Momose M, Tsukagoshi T, Dullemond, CP. 2016a. Ap. J. 820:54 [4.3.3]

Kataoka A, Tsukagoshi T, Momose M, et al. 2016b. Ap. J. Lett. 831:L12 [4.3.3]

Kataoka A, Okuzumi S, Tazaki R. 2019. Ap. J. Lett. 874:L6 [2.5]

Kenyon SJ, Hartmann L. 1987. Ap. J. 323:714-733 [2.4]

Keppler M, Benisty M, Müller A, et al. 2018. Astron. Astrophys. 617:A44 [5.4]

Klahr HH, Bodenheimer P. 2003, Ap. J. 582:869-892 [5.2.1]

—. 2006. Ap. J. 639:432-440 [5.2.1]

Klahr HH, Henning T. 1997. Icarus 128:213-229 [5.2.1]

Kley W, Dirksen G. 2006. Astron. Astrophys. 447:369-377 [5.2.2]

Kley W, Nelson RP. 2012. Annu. Rev. Astron. Astrophys. 50:211-249 [5.2.2]

Kratter K, Lodato G. 2016. Annu. Rev. Astron. Astrophys. 54:271-311 [5.2.1]

Kraus AL, Ireland MJ, Martinache F, Hillenbrand LA. 2011. Ap. J. 731:8 [3.2.1]

Kraus S, Kreplin A, Fukugawa M, et al. 2017. Ap. J. Lett. 848:L11 [5.3.1, 11]

Krijt S, Ormel CW, Dominik C, Tielens AGGM. 2015. Astron. Astrophys. 574:A83 [4.1]

Kudo T, Hashimoto J, Muto T, et al. 2018. Ap. J. Lett. 868:L5 [5.3.1, 11]

Kurtovic NT, Pérez LM, Benisty M, et al. 2018. Ap. J. Lett. 869:L44 [3.2.1]

Laibe G, Gonzalez JF, Maddison ST. 2012. Astron. Astrophys. 537:A61 [4.4]

Lambrechts M, Johansen A. 2012. Astron. Astrophys. 544:A32 [5.4]

Laughlin G, Bodenheimer P. 1994. Ap. J. 436:335-354 [5.2.1]

Lesur G, Hennebelle P, Fromang S. 2015. Astron. Astrophys. 582:L9 [5.2.1]

Li Z-Y, Banerjee R, Pudritz RE, et al. 2014. In Protostars and Planets VI, ed. H Beuther, RS

Klessen, CP Dullemond, T Henning, pp. 173-194. Tucson: Univ. Ariz. Press [1.1]

Lin DNC, Papaloizou J. 1979. MNRAS 186:799-812 [5.2.2]

—. 1986. Ap. J. 309:846-857 [5.2.2]

Liu HB. 2019. Ap. J. Lett. 877:L22 [4.3.4, 5.3.3]

Lodato G, Dipierro G, Ragusa E, et al. 2019. MNRAS 486:453-461 [13, 5.4] 
Lommen DJP, van Dishoeck EF, Wright CM, et al. 2010. Astron. Astrophys. 515:A77 [9]

Long F, Herczeg GJ, Pascucci I, et al. 2017. Ap. J. 844:99 [2.1]

-. 2018a, Ap. J. 863:61 [3]

Long F, Pinilla P, Herczeg GJ, et al. 2018b. Ap. J. 869:17 [5.3.1, 5.3.1, 11, 5.3.2, 5.4, 13]

Long F, Herczeg GJ, Harsono D, et al. 2019. Ap. J. 882:49 [6]

Loomis RA, Öberg KI, Andrews SM, MacGregor MA. 2017. Ap. J. 840:23 [5.3.1, 5.3.6]

Lorén-Aguilar P, Bate MR. 2015. MNRAS 453:L78-L82 [5.2.1]

—. 2016. MNRAS 457:L54-L58 [5.2.1]

Louvet F, Dougados C, Cabrit S, et al. 2018. Astron. Astrophys. 618:A120 [4.3.1]

Lovelace RVE, Li H, Colgate SA, et al. 1999. Ap. J. 513:805-810 [5.2.1]

Lynden-Bell D, Pringle JE. 1974. MNRAS 168:603-637 [2.5]

Lyra W, Johansen A, Zsom A, Klahr H, Piskunov N. 2009. Astron. Astrophys. 497:869-888 [5.2.1]

Lyra W, Lin MK. 2013. Ap. J. 775:17 [5.2.1]

Manara CF, Rosotti G, Testi L, et al. 2016. Astron. Astrophys. 591:L3 [2.1]

Manara CF, Morbidelli A, Guillot T. 2018. Astron. Astrophys. 618:L3 [2.1]

Manara CF, Tazzari M, Long F, et al. 2019. Astron. Astrophys. 628:A95 [6, 3.2.1]

Mann RK, Williams JP. 2009. Ap. J. Lett. 694:L36-L40 [3.2.2]

—. 2010. Ap. J. 725:430-442 [3.2.2]

Mann RK, Di Francesco J, Johnstone D, et al. 2014. Ap. J. 784:82 [3.2.2]

Marino S, Pérez S, Casassus S. 2015. Ap. J. Lett. 798:L44 [5.3.1, 12, 5.3.6]

Maury AJ, André P, Testi L, et al. 2019. Astron. Astrophys. 621:A76 [3.3]

Mayama S, Hashimoto J, Muto T, et al. 2012. Ap. J. Lett. 760:L26 [3.2.1, 5.3.1]

McCabe C, Duchêne G, Pinte C, et al. 2011. Ap. J. 727:90 [4.3.1]

McClure MK, Bergin EA, Cleeves LI, et al. 2016. Ap. J. 831:167 [2.1]

Menu J, van Boekel R, Henning T, et al. 2014. Astron. Astrophys. 564:A93 [4.3.2]

Miguel Y, Guilera OM, Brunini A. 2011. MNRAS 412:2113-2124 [2.3]

Min M, Rab C, Woitke P, Dominik C, Ménard F. 2016. Astron. Astrophys. 585:A13 [4.1]

Miotello A, Bruderer S, van Dishoeck EF. 2014. Astron. Astrophys. 572:A96 [2.1]

Miotello A, van Dishoeck EF, Kama M, Bruderer S. 2016. Astron. Astrophys. 594:A85 [2.1]

Miotello A, van Dishoeck EF, Williams JP, et al. 2017. Astron. Astrophys. 599:A113 [3, 2.1]

Miotello A, Facchini S, van Dishoeck EF, Bruderer S. 2018. Astron. Astrophys. 619:A113 [2.3]

Miyake K, Nakagawa Y. 1993. Icarus 106:20-41 [4.2]

Monnier JD, Harries TJ, Aarnio A, et al. 2017. Ap. J. 838:20 [11]

Monnier JD, Harries TJ, Bae J, et al. 2019. Ap. J. 872:122 [5.3.1]

Montesinos M, Pérez S, Casassus S, et al. 2016. Ap. J. Lett. 823:L8 [5.3.6]

Mulders GD, Dominik C. 2012. Astron. Astrophys. 539:A9 [4.3.1]

Mulders GD. 2018. In Handbook of Exoplanets, ed. H Deeg, J Belmonte, 153. Springer, Cham [3.1]

Müller A, Keppler M, Henning T, et al. 2018. Astron. Astrophys. 617:L2 [5.4]

Muro-Arena GA, Dominik C, Waters LBFM, et al. 2018. Astron. Astrophys. 614:A24 [4.3.1]

Muto T, Grady CA, Hashimoto J, et al. 2012. Ap. J. Lett. 748:L22 [5.3.1, 5.3.2]

Muzerolle J, Flaherty K, Balog Z, et al. 2009. Ap. J. Lett. 704:L15-L19 [5.3.6]

Najita JR, Kenyon SJ. 2014. MNRAS 445:3315-3329 [2.1]

Nakagawa Y, Sekiya M, Hayashi C. 1986. Icarus 67:375-390 [4.1]

Natta A. 1993. Ap. J. 412:761-770 [2.4]

Nealon R, Dipierro G, Alexander R, Martin RG, Nixon C. 2018. MNRAS 481:20-35 [5.2.2]

Nealon R, Pinte C, Alexander R, Mentiplay D, Dipierro G. 2019. MNRAS 484:4951-4962 [5.3.6]

Nixon CJ, King AR, Pringle JE. 2018. MNRAS 477:3273-3278 [2.1]

Öberg KI, Qi C, Fogel JKJ, et al. 2011. Ap. J. 734:98 [4]

Ohashi S, Kataoka A, Nagai H, et al. 2018. Ap. J. 864:81 [4.3.3]

Okuzumi S, Tanaka H, Kobayashi H, Wada K. 2012. Ap. J. 752:106 [4.4]

Okuzumi S, Momose M, Sirono S-i, Kobayashi H, Tanaka H. 2016. Ap. J. 821:82 [5.2.3] 
Ormel CW, Klahr HH. 2010. Astron. Astrophys. 520:A43 [5.4]

Owen JE, Lai D. 2017. MNRAS 469:2834-2844 [5.2.2]

Paardekooper S-J, Mellema G. 2006. Astron. Astrophys. 453:1129-1140 [5.2.2]

Pan L, Padoan P, Scalo J, et al. 2011. Ap. J. 740:6 [5.2.1]

Panić O, Hogerheijde MR, Wilner D, Qi C. 2009. Astron. Astrophys. 501:269-278 [2.2, 4.3.5]

Pascucci I, Testi L, Herczeg GJ, et al. 2016. Ap. J. 831:125 [3, 3.1, 3.1, 3.3]

Pérez LM, Carpenter JM, Chandler CJ, et al. 2012. Ap. J. Lett. 760:L17 [4.3.2]

Pérez LM, Isella A, Carpenter JM, Chandler CJ. 2014. Ap. J. Lett. 783:L13 [5.3.1]

Pérez LM, Chandler CJ, Isella A, et al. 2015a. Ap. J. Lett. 813:41 [4.3.2]

Pérez S, Dunhill A, Casassus S, et al. 2015b. Ap. J. Lett. 811:L5 [5.3.5]

Pérez LM, Carpenter JM, Andrews SM, et al. 2016. Science 353:1519-1521 [5.3.1]

Pérez S, Casassus S, Benítez-Llambay P. 2018a. MNRAS 480:L12-L17 [5.3.5]

Pérez LM, Benisty M, Andrews SM, et al. 2018b. Ap. J. Lett. 869:L50 [5.3.1, 11]

Pérez S, Casassus S, Baruteau C, et al. 2019a. Astron. J. 158:15 [11]

Pfalzner S. 2013. Astron. Astrophys. 549:A82 [3.2.1]

Piétu V, Dutrey A, Guilloteau S. 2007. Astron. Astrophys. 467:163-178 [2.3, 5.3.1]

Piétu V, Guilloteau S, Di Folco E, Dutrey A, Boehler Y. 2014. Astron. Astrophys. 564:A95 [2.2, 2.3]

Pinilla P, Birnstiel T, Ricci L, et al. 2012a. Astron. Astrophys. 538:A114 [5.1]

Pinilla P, Benisty M, Birnstiel T. 2012b. Astron. Astrophys. 545:A81 [5.3, 5.3.1]

Pinilla P, Birnstiel T, Benisty M, et al. 2013. Astron. Astrophys. 554:A95 [4.4, 5.1]

Pinilla P, Pérez LM, Andrews S, et al. 2017a. Ap. J. 839:99 [11]

Pinilla P, Pohl A, Stammler SM, Birnstiel T. 2017b. Ap. J. 845:68 [5.2.3]

Pinilla P, Quiroga-Nuñez LH, Benisty M, et al. 2017c. Ap. J. 846:70 [9]

Pinilla P, Natta A, Manara CF, et al. 2018a. Astron. Astrophys. 615:A95 [5.3.2]

Pinilla P, Tazzari M, Pascucci I, et al. 2018b. Ap. J. 859:32 [5.3.1, 11, 5.3.2, 5.4]

Pinilla P, Benisty M, de Boer J, et al. 2018c. Ap. J. 868:85 [11, 5.3.6, 5.3.6]

Pinte C, Dent WRF, Ménard F, et al. 2016. Ap. J. 816:25 [2.5, 4.3.2]

Pinte C, Fouchet L, Ménard F, Gonzalez J-F, Duchêne G. 2007. Astron. Astrophys. 469:963-971 [4.3.1]

Pinte C, Padgett DL, Ménard F, et al. 2008. Astron. Astrophys. 489:633-650 [2.4]

Pinte C, Ménard F, Duchêne G, et al. 2018a. Astron. Astrophys. 609:A47 [2.4]

Pinte C, Price DJ, Ménard F, et al. 2018b. Ap. J. Lett. 860:L13 [5.3.5]

Pinte C, van der Plas G, Ménard F, et al. 2019. Nature Ast. 459 [5.3.5, 5.4]

Pollack JB, Hollenbach D, Beckwith S, et al. 1994. Ap. J. 421:615-639 [4.2]

Powell D, Murray-Clay R, Schlichting HE. 2017. Ap. J. 840:93 [2.3]

Qi C, D'Alessio P, Öberg KI, et al. 2011. Ap. J. 740:84 [2.4]

Qi C, Öberg KI, Espaillat CC, et al. 2019. Ap. J. 882:160 [2.4]

Quanz SP, Avenhaus H, Buenzli E, et al. 2013. Ap. J. Lett. 766:L2 [5.3.1]

Quanz SP, Schmid HM, Geissler K, et al. 2011. Ap. J. 738:23 [4.3.1]

Rafikov RR. 2016. Ap. J. 831:122 [2.4]

Raghavan D, McAlister HA, Henry TJ, et al. 2010. Ap. J. Suppl. 190:1-42 [3.2.1]

Rapson VA, Kastner JH, Andrews SM, et al. 2015. Ap. J. Lett. 803:L10 [5.3.1]

Reboussin L, Wakelam V, Guilloteau S, Hersant F, Dutrey A. 2015. Astron. Astrophys. 579:A82 [2.1]

Rebull LM, Cody AM, Covey KR, et al. 2014. Astron. J. 148:92 [5.3.6]

Regály Z, Juhász A, Sándor Z, et al. 2012. MNRAS 419:1701-1712 [5.2.1]

Ricci L, Testi L, Natta A, Brooks KJ. 2010a. Astron. Astrophys. 521:A66 [4.3.2, 9]

Ricci L, Testi L, Natta A, et al. 2010b. Astron. Astrophys. 512:A15 [4.2, 4.3.2, 9]

Ricci L, Trotta F, Testi L, et al. 2012. Astron. Astrophys. 540:A6 [3.1, 9, 4.3.4]

Ricci L, Isella A, Carpenter JM, Testi L. 2013. Ap. J. Lett. 764:L27 [9] 
Ricci L, Testi L, Natta A, et al. 2014. Ap. J. 791:20 [9]

Rice WKM, Lodato G, Pringle JE, Armitage PJ, Bonnell IA. 2004. MNRAS 355:543 [5.2.1]

Rice WKM, Armitage PJ, Wood K, Lodato G. 2006. MNRAS 373:1619-1626 [5.2.2]

Richard S, Nelson RP, Umurhan OM. 2016. MNRAS 456:3571-3584 [5.2.1]

Rodriguez JE, Loomis R, Cabrit S, et al. 2018. Ap. J. 859:150 [3.2.1]

Ros K, Johansen A. 2013. Astron. Astrophys. 552:A137 [5.2.3]

Ros K, Johansen A, Riipinen I, Schlesinger D. 2019. Astron. Astrophys. 629:A65 [5.2.3]

Rosenfeld KA, Andrews SM, Wilner DJ, Stempels HC. 2012a. Ap. J. 759:119 [2.5]

Rosenfeld KA, Qi C, Andrews SM, et al. 2012b. Ap. J. 757:129 [5.3.6]

Rosenfeld KA, Andrews SM, Hughes AM, Wilner DJ, Qi C. 2013a. Ap. J. 774:16 [2.4, 2.5]

Rosenfeld KA, Chiang E, Andrews SM. 2014. Ap. J. 782:62 [5.3.6]

Rosotti GP, Dale JE, de Juan Ovelar M, et al. 2014. MNRAS 441:2094-2110 [3.2.1]

Rosotti GP, Juhasz A, Booth RA, Clarke CJ. 2016. MNRAS 459:2790-2805 [5.2.2]

Rosotti GP, Booth RA, Tazzari M, et al. 2019a. MNRAS 486:L63-L68 [2.2, 3.3, 4.4]

Rosotti GP, Tazzari M, Booth RA, et al. 2019b. MNRAS 486:4829-4844 [2.2, 4.3.5]

Ruge JP, Flock M, Wolf S, et al. 2016. Astron. Astrophys. 590:A17 [5.4]

Ruíz-Rodríguez D, Cieza LA, Williams JP, et al. 2018. MNRAS 478:3674-3692 [3, 3.3]

Saito E, Sirono S-i. 2011. Ap. J. 728:20 [5.2.3]

Schneider G, Wood K, Silverstone MD, et al. 2003. Astron. J. 125:1467-1479 [4.3.1]

Schoonenberg D, Ormel CW. 2017. Astron. Astrophys. 602:A21 [5.2.3]

Schwarz KR, Bergin EA, Cleeves LI, et al. 2016. Ap. J. 823:91 [2.3, 2.4]

Segura-Cox DM, Looney LW, Tobin JJ, et al. 2018. Ap. J. 866:161 [3.3]

Shariff K, Cuzzi JN. 2011. Ap. J. 738:73 [5.2.1]

Sheehan PD, Eisner JA. 2017. Ap. J. 851:45 [3.3]

Shi JM, Chiang E. 2014. Ap. J. 789:34 [2.5]

Simon JB, Armitage PJ. 2014. Ap. J. 784:15 [5.2.1]

Simon M, Guilloteau S, Di Folco E, et al. 2017. Ap. J. 844:158 [4, 2.5]

Sirono S-i. 2011. Ap. J. 735:131 [5.2.3]

Stammler SM, Birnstiel T, Panić O, Dullemond CP, Dominik C. 2017. Astron. Astrophys. 600:A140 $[5.2 .3]$

Stammler SM, Drążkowska J, Birnstiel T, et al. 2019 Ap. J. Lett. 884:L5 [5.3.3]

Stephens IW, Yang H, Li Z-Y, et al. 2017. Ap. J. 851:55 [4.3.3]

Stevenson DJ, Lunine JI. 1988. Icarus. 75:146-155 [5.2.3]

Stolker T, Dominik C, Min M, et al. 2016. Astron. Astrophys. 596:A70 [5.3.1, 5.3.2, 5.3.6]

Stolker T, Sitko M, Lazareff B, et al. 2017. Ap. J. 849:143 [11, 5.3.6]

Störzer H, Hollenbach D. 1999. Ap. J. 515:669-684 [3.2.2]

Suriano SS, Li Z-Y, Krasnopolsky R, Shang H. 2017. MNRAS 468:3850-3868 [5.2.1]

—. 2018. MNRAS 477:1239-1257 [5.2.1]

Surville C, Mayer L, Lin DNC. 2016. Ap. J. 831:82 [5.2.1]

Suzuki TK, Ogihara M, Morbidelli AR, Crida A, Guillot T. 2016. Astron. Astrophys. 596:A74 [5.2.1]

Takahashi SZ, Inutsuka S-i. 2014. Ap. J. 794:55 [5.2.1]

-. 2016. Astron. J. 152:184 [5.2.1]

Takahashi SZ, Muto T. 2018 Ap. J. 865:102 [5.2.1]

Takeuchi T, Lin DNC. 2002. Ap. J. 581:1344-1355 [4.1, 5.1]

- 2005. Ap. J. 623:482-492 [4.1, 5.1]

Tang Y-W, Guilloteau S, Piétu V, et al. 2012. Astron. Astrophys. 547:A84 [5.3.1]

Tang Y-W, Guilloteau S, Dutrey A, et al. 2017. Ap. J. 840:32 [5.3.1]

Tazaki R, Lazarian A, Nomura H. 2017. Ap. J. 839:56 [2.5]

Tazzari M, Testi L, Ercolano B, et al. 2016. Astron. Astrophys. 588:A53 [9, 4.3.2]

Tazzari M, Testi L, Natta A, et al. 2017. Astron. Astrophys. 606:A88 [2.3, 3.1]

Teague R, Bae J, Bergin EA, Birnstiel T, Foreman-Mackey D. 2018a. Ap. J. Lett. 860:L12 [5.3.5, 
$5.4]$

Teague R, Bae J, Birnstiel T, Bergin EA. 2018b. Ap. J. 868:113 [2.5, 5.3.5, 5.4]

Teague, R, Bae, J, Huang, J, Bergin EA. 2019. Ap. J. Lett. in press [5.3.1]

Teague R, Henning T, Guilloteau S, et al. 2018. Ap. J. 864:133 [2.3]

Teague R, Guilloteau S, Semenov D, et al. 2016. Astron. Astrophys. 592:A49 [2.5]

Teiser J, Wurm G. 2009. MNRAS 393:1584-1594 [4.1]

Terebey S, Shu FH, Cassen P. 1984. Ap. J. 286:529-551 [1.1]

Testi L, Birnstiel T, Ricci L, et al. 2014. In Protostars and Planets VI, ed. H Beuther, RS Klessen,

CP Dullemond, T Henning, pp. 339-361. Tucson: Univ. Ariz. Press [4.1]

Testi L, Natta A, Scholz A, et al. 2016. Astron. Astrophys. 593:A111 [9]

Thamm E, Steinacker J, Henning T. 1994. Astron. Astrophys. 287:493-502 [2.4]

Tobin JJ, Looney LW, Wilner DJ, et al. 2015. Ap. J. 805:125 [3.3]

Tobin JJ, Kratter KM, Persson MV, et al. 2016. Nature 538:483-486 [3.2.1]

Tomida K, Machida MN, Hosokawa T, Sakurai Y, Lin CH. 2017. Ap. J. Lett. 835:L11 [5.2.1]

Toomre A. 1964. Ap. J. 139:1217-1238 [5.2.1]

Trapman L, Facchini S, Hogerheijde MR, van Dishoeck EF, Bruderer S. 2019. Astron. Astrophys. 629:A79 [2.2, 4.3.5]

Trapman L, Miotello A, Kama M, van Dishoeck EF, Bruderer S. 2017. Astron. Astrophys. 605:A69 [2.1]

Tripathi A, Andrews SM, Birnstiel T, Wilner DJ. 2017. Ap. J. 845:44 [2.2, 4, 3.1, 3.3, 4.4, 5.3.3]

Tripathi A, Andrews SM, Birnstiel T, et al. 2018. Ap. J. 861:64 [9, 4.3.2, 4.4]

Tsukagoshi T, Nomura H, Muto T, et al. 2016. Ap. J. Lett. 829:L35 [5.3.3]

Tsukagoshi T, Momose M, Kitamura Y, et al. 2019a. Ap. J. 871:5 [11]

Tsukagoshi T, Muto T, Nomura H, et al. 2019b. Ap. J. Lett. 878:L8 [5.3.2]

Turner NJ, Carballido A, Sano T. 2010. Ap. J. 708:188-201 [5.3.6]

Turner NJ, Fromang S, Gammie C, et al. 2014. In Protostars and Planets VI, ed. H Beuther, RS

Klessen, CP Dullemond, T Henning, pp. 411-432. Tucson: Univ. Ariz. Press [2.5]

Tychoniec L, Tobin JJ, Karska A, et al. 2018. Ap. J. Suppl. 238:19 [3.3]

Uribe AL, Klahr H, Flock M, Henning T. 2011. Ap. J. 736:85 [5.2.1]

van Boekel R, Waters LBFM, Dominik C, et al. 2004. Astron. Astrophys. 418:177-184 [4.3.1]

van Boekel R, Henning T, Menu J, et al. 2017. Ap. J. 837:132 [2]

van der Marel N, Cazzoletti P, Pinilla P, Garufi A. 2016a. Ap. J. 832:178 [5.3.1]

van der Marel N, Dong R, Di Francesco J, Williams JP, Tobin J. 2019. Ap. J. 872:112 [5.3.2, 5.4]

van der Marel N, Pinilla P, Tobin J, et al. 2015a. Ap. J. Lett. 810:L7 [5.4]

van der Marel N, van Dishoeck E, Bruderer S, Pérez LM, Isella A. 2015b. Astron. Astrophys.

579:A106 [5.3.1, 5.3.2, 5.4]

van der Marel N, van Dishoeck EF, Bruderer S, et al. 2016b. Astron. Astrophys. 585:A58 [5.3.1, $5.3 .2,5.4]$

van der Marel N, Verhaar BW, van Terwisga S, et al. 2016c. Astron. Astrophys. 592:A126 [5.3.1]

van der Marel N, Williams JP, Bruderer S. 2018a. Ap. J. Lett. 867:L14 [5.4]

van der Marel N, van Dishoeck EF, Bruderer S, et al. 2013. Science 340:1199-1202 [5.3.1, 5.3.2]

van der Marel N, Williams JP, Ansdell M, et al. 2018b. Ap. J. 854:177 [11]

van der Plas G, Ménard F, Ward-Duong K, et al. 2016. Ap. J. 819:102 [9]

van der Plas G, Ménard F, Canovas H, et al. 2017. Astron. Astrophys. 607:A55 [11]

van der Walt S, Colbert SC, Varoquaux G. 2011. Comp. in Sci. and Eng. 13:22 [6]

van Terwisga SE, van Dishoeck EF, Ansdell M, et al. 2018. Astron. Astrophys. 616:A88 [11, 5.3.2, $5.4]$

van Terwisga SE, Hacar A, van Dishoeck EF. 2019. Astron. Astrophys. 628:A85 [3.2.2]

van Zadelhoff G-J, van Dishoeck EF, Thi W-F, Blake GA. 2001. Astron. Astrophys. 377:566-580

$[2.1,2.3,2.4]$

Villenave M, Benisty M, Dent WRF, et al. 2019. Astron. Astrophys. 624:A7 [5.3.1, 11] 
Ward-Duong K, Patience J, Bulger J, et al. 2018. Astron. J. 155:54 [3.1, 9]

Weaver E, Isella A, Boehler J. 2018. Ap. J. 853:113 [2.3, 2.4, 5.3.3]

Weidenschilling SJ. 1977a. Astrophys. Space Sci. 51:153-158 [2.1, 3]

—. 1977b. MNRAS 180:57-70 [2.5, 4.1]

Weinberger AJ, Becklin EE, Schneider G, et al. 2002. Ap. J. 566:409-418 [4.3.1]

Whipple FL. 1972. In From Plasma to Planet, ed. A Elvius, pp. 211-232. New York: Wiley [4.1]

Williams JP, Best WMJ. 2014. Ap. J. 788:59 [2.1]

Williams JP, Cieza L, Hales A, et al. 2019. Ap. J. Lett. 875:L9 [3, 3.3]

Williams JP, Cieza LA. 2011. Annu. Rev. Astron. Astrophys. 49:67-117 [3.3]

Williams JP, McPartland C. 2016. Ap. J. 830:32 [2.3]

Williams JP, Mann RK, Di Francesco J, et al. 2014. Ap. J. 796:120 [3.2.1]

Windmark F, Birnstiel T, Güttler C, et al. 2012a. Astron. Astrophys. 540:A73 [4.4]

Windmark F, Birnstiel T, Ormel CW, Dullemond CP. 2012b. Astron. Astrophys. 544:L16 [4.4]

Winter AJ, Booth RA, Clarke CJ. 2018a. MNRAS 479:5522-5531 [3.2.1]

Woitke P, Min M, Pinte C, et al. 2016. Astron. Astrophys. 586:A103 [4.1]

Wünsch R, Klahr H, Różyczka M. 2005. MNRAS 362:361-368 [5.2.1]

Xiang-Gruess M. 2016. MNRAS 455:3086-3100 [3.2.1]

Yang CC, Johansen A, Carrera D. 2017a. Astron. Astrophys. 606:A80 [5.1]

Yang H, Li Z-Y, Looney LW, Girart JM, Stephens IW. 2017b. MNRAS 472:373-388 [4.3.3]

Youdin AN. 2011. Ap. J. 731:99 [5.2.1]

Youdin AN, Goodman J. 2005. Ap. J. 620:459-469 [5.1]

Youdin AN, Shu FH. 2002. Ap. J. 580:494-505 [5.1]

Yu M, Willacy K, Dodson-Robinson SE, Turner NJ, Evans NJ. 2016. Ap. J. 822:53 [2.1]

Yu M, Evans NJ, Dodson-Robinson SE, Willacy K, Turner NJ. 2017. Ap. J. 841:39 [2.1]

Yu S-Y, Ho LC, Zhu Z. 2019. Ap. J. 877:100 [5.3.2]

Zhang K, Bergin EA, Blake GA, et al. 2016. Ap. J. Lett. 818:L16 [5.3.1]

Zhang K, Bergin EA, Blake GA, Cleeves LI, Schwarz, KR. 2017 Nature Astronomy 1:0130 [2.3]

Zhang K, Bergin EA, Schwarz KR, et al. 2019. Ap. J. 883:98 [2.3]

Zhang K, Blake GA, Bergin EA. 2015. Ap. J. Lett. 806:L7 [5.4]

Zhang S, Zhu Z, Huang J, et al. 2018. Ap. J. Lett. 869:L47 [5.3.5, 13, 5.4]

Zhu Z, Dong R, Stone JM, Rafikov RR. 2015. Ap. J. 813:88 [5.2.2]

Zhu Z, Nelson RP, Dong R, Espaillat C, Hartmann L. 2012. Ap. J. 755:6 [5.2.2, 5.4]

Zhu Z, Nelson RP, Hartmann L, Espaillat C, Calvet N. 2011. Ap. J. 729:47 [5.2.2]

Zhu Z, Stone JM, Rafikov RR, Bai X-N. 2014. Ap. J. 785:122 [5.2.2]

Zhu Z, Zhang S, Jiang YF, et al. 2019. Ap. J. Lett. 877:L18 [2.1, 2.2, 3.1, 4.3.4, 5.3.3]

Zhu Z. 2019. MNRAS 483:4221-4241 [5.2.2]

Zsom A, Ormel CW, Güttler C, Blum J, Dullemond CP. 2010. Astron. Astrophys. 513:A57 [4.1] 